\title{
Tensor models, Kronecker coefficients and permutation centralizer algebras
}

\author{
Joseph Ben Geloun ${ }^{a, c}$ and Sanjaye Ramgoolam ${ }^{b, d}$ \\ ${ }^{a}$ Laboratoire d'Informatique de Paris Nord UMR CNRS 7030, Université Paris 13, \\ 99, avenue J.-B. Clement, 93430 Villetaneuse, France \\ ${ }^{b}$ School of Physics and Astronomy, Centre for Research in String Theory, \\ Queen Mary University of London, \\ London E1 4NS, United Kingdom \\ ${ }^{c}$ International Chair in Mathematical Physics and Applications, ICMPA-UNESCO Chair, 072, \\ B.P. 50 Cotonou, Benin \\ ${ }^{d}$ School of Physics and Mandelstam Institute for Theoretical Physics, University of Witwatersrand, \\ Wits, 2050, South Africa \\ E-mail: bengeloun@lipn.univ-paris13.fr, s.ramgoolam@qmul.ac.uk
}

ABSTRACT: We show that the counting of observables and correlators for a 3-index tensor model are organized by the structure of a family of permutation centralizer algebras. These algebras are shown to be semi-simple and their Wedderburn-Artin decompositions into matrix blocks are given in terms of Clebsch-Gordan coefficients of symmetric groups. The matrix basis for the algebras also gives an orthogonal basis for the tensor observables which diagonalizes the Gaussian two-point functions. The centres of the algebras are associated with correlators which are expressible in terms of Kronecker coefficients (Clebsch-Gordan multiplicities of symmetric groups). The color-exchange symmetry present in the Gaussian model, as well as a large class of interacting models, is used to refine the description of the permutation centralizer algebras. This discussion is extended to a general number of colors $d$ : it is used to prove the integrality of an infinite family of number sequences related to color-symmetrizations of colored graphs, and expressible in terms of symmetric group representation theory data. Generalizing a connection between matrix models and Belyi maps, correlators in Gaussian tensor models are interpreted in terms of covers of singular 2-complexes. There is an intriguing difference, between matrix and higher rank tensor models, in the computational complexity of superficially comparable correlators of observables parametrized by Young diagrams.

KEYwords: 1/N Expansion, AdS-CFT Correspondence, Gauge-gravity correspondence, Discrete Symmetries

ARXIV EPRINT: 1708.03524 


\section{Contents}

1 Introduction 1

2 Observables in tensor models using permutations 5

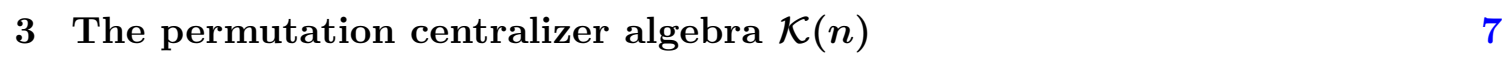

$\begin{array}{lll}3.1 & \text { Counting observables and Kronecker coefficients } & 7\end{array}$

$\begin{array}{lll}3.2 & \mathcal{K}(n) \text { as a centralizer algebra in } \mathbb{C}\left(S_{n}\right) \otimes \mathbb{C}\left(S_{n}\right) & 10\end{array}$

3.3 The centre $\mathcal{Z}(\mathcal{K}(n))$ of $\mathcal{K}(n) \quad 13$

$\begin{array}{lll}3.4 & \text { Double coset algebra } & 15\end{array}$

$\begin{array}{lll}4 \mathcal{K}(n) \text { as a graph algebra } & 18\end{array}$

5 PCAs and correlators $\quad 21$

5.1 Correlators for central observables 21

5.2 Correlators and Kronecker coefficients: explicit examples 24

5.3 Orthogonality of two-point functions and WA basis for $\mathcal{K}(n) \quad 27$

$\begin{array}{lll}5.4 \mathcal{K}^{\infty} \text { and correlators } & 29\end{array}$

6 Correlators, permutation-TFT2 and covers of 2-complexes 30

$\begin{array}{lll}\mathbf{7} & \boldsymbol{S}_{\mathbf{3}} \text {-color exchange symmetry } & \mathbf{3 4}\end{array}$

7.1 Counting observables in the rank $d=3$ case $\quad 35$

$\begin{array}{lll}7.2 & \text { The algebra } \mathcal{K}_{Y_{0}}(n) & 36\end{array}$

7.3 Decomposition of $\mathcal{K}(n)$ into representations of $S_{3} \quad 36$

$\begin{array}{lll}7.4 & \text { Color symmetrisation using Fourier basis } & 39\end{array}$

7.4.1 Subspace of $\mathcal{K}(n)$ with $R_{1,2,3}$ all different $\quad 40$

7.4.2 Subspace of $\mathcal{K}(n)$ where two $S_{n}$ irreps are equal and different from third 41

7.4.3 Subspace of $\mathcal{K}(n)$ with all equal $R_{1}=R_{2}=R_{3}=R \quad 43$

7.4.4 More on $S_{[2,1]}^{(3)}(n)$ and the character table of $S_{n} \quad 44$

7.5 $S_{3}$-refinement for $\mathcal{K}(n) \quad 46$

7.6 Counting color-symmetrised tensor invariants for general $d \quad 48$

$\begin{array}{lll}7.7 \mathcal{K}^{\infty} \text { and color symmetry } & 49\end{array}$

8 Summary and discussion $\quad 50$

$\begin{array}{lll}8.1 \text { Summary } & 50\end{array}$

8.2 Towards Young diagram statistical models and field theory 51

8.3 The space of holographic duals of tensor models 52

8.4 Computational complexity of central correlators in matrix versus tensor models 53

A Symmetric group, representation theory and group algebra $\quad 54$

$\begin{array}{lll}\text { A.1 Symmetric group and representation } & 54\end{array}$

$\begin{array}{lll}\text { A.2 Clebsch-Gordan coefficients } & 56\end{array}$

A.3 Projectors for irreducible representations $\quad 60$ 
B The permutation centralizer algebra $\mathcal{K}(n)$

B.1 Basis of $\mathcal{K}(n) \quad 60$

B.2 Basis of $\mathcal{Z}(\mathcal{K}(n)) \quad 64$

C Multiplication table of rank $d=3, n=3$ colored tensor graphs $\quad 65$

$\begin{array}{ll}\text { D Correlators of tensor observables } & 66\end{array}$

\section{Introduction}

Introduced as generalizations of matrix models [1,2] to study the discrete-to-continuum transition for discretized path integrals in quantum gravity, tensor models [3-5] and their further generalizations [6] were found to be tremendously more difficult to handle than the theory of matrices. One the main sources of difficulties in the study of tensor models at that time was the absence of an organizing principle for their partition function. Matrix models are organized by the large $N$ expansion [7] which sorts maps by their genus, and typically a world-sheet 't Hooft coupling constant at fixed genus. After approximately two decades, significant progress on tensor models emerged in a series of papers [8-13]. The large $N$ expansion for colored tensors was characterized in terms of sets of ribbon graphs known as "jackets" and new double scaling limits involving "melons" were found. Since then, many results on random tensors [14] have been achieved from statistical mechanics, to quantum field theory but as well in combinatorics and probability theory (see [15-18] and the reviews [19-21] and [22]). Recently, the large $N$ expansion for tensors added another twist in this already-remarkable story: the large $N$ limit of the famous Sachdev-YeKitaev (SYK) condensed matter model [23-27] matches with the same limit of a quantum mechanical model built with colored tensors without disorder [28, 29]. The SYK model is an active topic of research, its connections being explored with black hole physics, AdS/CFT correspondence, quantum gravity and condensed matter physics. The new connection between tensor and SYK models has thus come to be of relevance to several areas of theoretical physics (see for instance [30,33-38] and references therein).

A better understanding of the combinatorics of tensor models will be crucial in identifying and characterizing their holographic duals. There are two closely related aspects to the combinatorics, in the first instance the enumeration of observables, in the second, the understanding of the correlators. The former has immediate implications for thermodynamic questions related to these models (for recent investigations focused on these aspects see [39]), the spectrum of physical excitations in the holographic duals and since observables can be used to parametrize deformations of a given model, for the space of possible holographic duals. The implications of the counting for the intricacy of the holographic dual has been discussed in [30], see also [31, 32]. In the context of the AdS/CFT correspondence, correlators in the gauge theory or matrix models provide refined information about the holographic dual: interactions of gravitons, strings and branes. From a mathematical point of view, they are related to a host of interesting algebraic structures, notably integrability and hidden symmetries [40]. 
In the paper [41], we developed a variety of counting formulae starting from the complete set of invariant observables in tensor models with a complex tensor field having $d$ indices transforming in the fundamental of $\mathrm{U}(N)^{\times d}$. The invariant observables are constructed from $n$ copies of the complex field $\Phi$ as well as $n$ copies of the conjugate field $\bar{\Phi}$. They are in 1-1 correspondence with graphs with two types of vertices, one for the $\Phi$ 's and one for the $\bar{\Phi}$ 's, and colored edges, one for each type of index. The invariants are constructed by contracting the indices in the fundamental of each $\mathrm{U}(N)$ with the indices in the anti-fundamental. They are parametrized by a sequence of $d$ permutations, each in $S_{n}$, one for each factor in the unitary symmetry group. These permutations are subject to equivalence relations, which characterize permutation sequences corresponding to the same invariant operator. It was observed that the counting formulae can be expressed in terms of topological field theory based on symmetric groups. The permutation description was used to give formulae for "normal-ordered correlators" in Gaussian tensor models.

An important additional symmetry exists in these Gaussian models, and indeed in a large class of interacting models. It is the group of all permutations of the $d$ types of indices, which form the symmetric group $S_{d}$. It had already been understood in the tensor model literature that the counting of the above tensor invariants can be expressed as a counting of colored graphs. It had also been understood that by taking advantage of the $S_{d}$ symmetry, it is useful to consider "color-symmetrized graphs" which are defined by additional equivalences generated by the $S_{d}$ action of color-exchanges on the graphs. In [41] we found counting formulae for the "color-symmetrized graphs" in terms of the permutation tuples. These generated sequences of positive integers. Intriguingly the formulae we obtained for the color symmetrized graphs were expressed as fractional sums of expressions, which turned out to themselves be integers. These sequences were denoted by $S_{p}^{(d)}(n)$ : specifying an integer $d$, along with a partition of $d$, we have a sequence of integers as $n$ ranges over positive integers. One of the results which follows from the detailed treatment of color exchange symmetry in this paper is to explain the integrality of these additional sequences, and give general expressions for them for any $d$ and $p \vdash d$.

The permutation approach to counting and correlators has been used in a number of papers in the context of AdS/CFT. It was used in the half-BPS sector in $[42,43]$ to find orthogonal bases for operators, which are useful in identifying CFT duals of giant gravitons. Following investigations of strings attached to giant gravitons in [44], orthogonal bases for multi-matrix operators in CFT were found in [45-50]. The key idea is to parametrize gauge invariants using permutations subject to equivalences, and to understand these equivalence classes using Fourier transformation on symmetric groups based on representation theory, to go from the equivalence classes of permutations to representation theoretic bases. A short review is in [51]. It has been realized that an important role in understanding these orthogonal bases is played by permutation centralizer algebras (PCAs) [52]. The basic observation is that the once we have found a formulation of a counting of invariants in terms of permutations subject to equivalences, it is useful to go to the group algebras of permutations and consider their sub-algebras associated to the equivalence classes. These algebras are semi-simple, i.e. they are associative and have a non-degenerate bilinear pairing. These properties are inherited from the underlying permutation group algebras. As 
a result, from the Wedderburn-Artin (WA) theorem [53, 54] on the structure of these algebras, we have a matrix decomposition of these algebras. The construction of orthogonal bases is, in many cases studied so far, closely connected to the workings of the WA theorem. This was studied in depth for the 2-matrix problem in [52] (closely related developments from the perspective of open-closed topological field theory are in $[55,56])$. In particular, the important role of the centre of the PCA was noted, in identifying a sector of correlators which are computable using characters, without requiring more refined representation theoretic quantities. The appropriate PCA for tensor model counting was identified and a basis in terms of Clebsch-Gordan coefficients of $S_{n}$ was described. It was called $\mathcal{K}(n)$ and its dimension was shown to be the sum of squares of Kronecker coefficients.

In this paper, we present a systematic study of $\mathcal{K}(n)$ and highlight the role of its structure, particularly in connection with the WA theorem, in correlators and orthogonal bases for tensor models. The role of color-exchange symmetry in the structure of $\mathcal{K}(n)$ is another important theme, which leads to new results on the integer sequences, denoted $S_{p}^{(d)}(n)$, which arose among the counting of color-symmetrized invariants in [41]. A key result of this paper is the formula for the dimension of the color-symmetrized sub-algebra of $\mathcal{K}(n)$ as a sum of squares of representation-theoretic quantities.

The paper is organized as follows. In section 2, we introduce the tensor model we will be discussing, based on complex tensors with $d$ indices transforming under $\mathrm{U}(N)^{\times d}$. We review the permutation approach to tensor models from [41].

The algebras $\mathcal{K}(n)$ are defined in section 3 . There are two equivalent descriptions of the algebra. In one description, they are sub-algebras of the tensor products $\mathbb{C}\left(S_{n}\right) \otimes \mathbb{C}\left(S_{n}\right)$ which commute with the diagonally embedded $\mathbb{C}\left(S_{n}\right)$. In equivalent terminology, $\mathcal{K}(n)$ is the centralizer of the diagonal $\mathbb{C}\left(S_{n}\right)$ in the tensor product $\mathbb{C}\left(S_{n}\right) \otimes \mathbb{C}\left(S_{n}\right)$, hence the name "permutation centralizer algebra" (PCA). Another type of PCA has been found to underlie a variety of results on correlators for the 2-matrix problem [52]: they are thus emerging as fundamental to the application of permutation group and representation theory techniques to matrix/tensor correlators. In the other description, they are sub-algebras of $\mathbb{C}\left(S_{n}\right) \otimes \mathbb{C}\left(S_{n}\right) \otimes \mathbb{C}\left(S_{n}\right)$ which are invariant under left and right diagonal actions. Both descriptions are based on the fact that tensor invariants can be described by sequences of permutations, subject to equivalences defined in terms of group multiplications in $S_{n}$. The second description is an algebra structure on a space of double cosets, so that $\mathcal{K}(n)$ is a double coset algebra. By partially gauge fixing the equivalences in the double coset description, we arrive at the PCA description. $\mathcal{K}(n)$ is a semi-simple associative algebra. As a result of the WA theorem, such algebras are isomorphic to direct sums of matrix algebras. We describe this direct sum decomposition of $\mathcal{K}(n)$. We can think of $\mathcal{K}(n)$ as made of matrix blocks. The terms in the sum are labelled by triples of Young diagrams with $n$ boxes, with non-vanishing Kronecker coefficient. These are triples $R_{1}, R_{2}, R_{3}$ of representations of $S_{n}$ such that the tensor product $R_{1} \otimes R_{2} \otimes R_{3}$ contains the trivial under the action of the diagonal $S_{n}$. The algebra elements belonging to the matrix block labelled by the ordered triple $\left[R_{1}, R_{2}, R_{3}\right]$, along with more refined data associated with the Kronecker multiplicities, are constructed using Clebsch-Gordan coefficients of the symmetric group. These are denoted $Q_{\tau_{1}, \tau_{2}}^{R_{1}, R_{2}, R_{3}}$. $\mathcal{K}(n)$ is a non-commutative algebra for generic $n$, so that 
the centre $\mathcal{Z}(\mathcal{K}(n))$ of $\mathcal{K}(n)$ is a proper subspace. Triples of Young diagrams label an overcomplete basis for the centre and triples with non-vanishing Kronecker coefficient label a basis.

$\mathcal{K}(n)$ also has an interpretation as a graph algebra. We explain this in section 4 . The identity of $\mathcal{K}(n)$ is the maximally disconnected melonic graph. For the lower orders $n \leq 4$, this algebra turns out to be commutative. We give illustrations of this algebra at $n=2$ and $n=3$ (multiplication tables). At $n=3$, there are elements which could factorize in different ways and this might lead to interesting properties.

Section 5 shows how the structure of $\mathcal{K}(n)$ described in section 3 organizes the properties of correlators in the Gaussian model. We consider two types of correlators: two-point functions of normal-ordered invariants, and one-point functions without normal ordering. We develop explicit formulae using known results on Kronecker coefficients for specific Young diagrams. The discussion of correlators makes it natural to consider the PCAs $\mathcal{K}(n)$ for all $n$ at once, where $n$ labels the number of $\Phi$ and $\bar{\Phi}$ in the invariant. Hence we consider and discuss the algebra

$$
\mathcal{K}^{\infty}=\bigoplus_{n=0}^{\infty} \mathcal{K}(n)
$$

using the convention that at $n=0, \mathcal{K}(0)=\mathbb{C}$.

In section 6 , we describe how tensor model correlators can be described by twodimensional topological field theory of permutations on 2-complexes. The permutationTFT2 description of counting and correlators for matrix theories is reviewed and applied to general quiver gauge theories in [57]. Some results on the connection between counting of tensor invariants and permutation-TFT2 were given in [41]. Here we consider correlators of these invariants as amplitudes in permutation TFT2.

In section 7 , we use the color-exchange symmetry $S_{3}$ of the rank-3 tensor model in order to give a refined description of $\mathcal{K}(n)$ in terms of irreducible representations (irreps) of $S_{3}$. We prove integrality of some sequences of numbers, which were observed in [41] but not proved. The subspace invariant under color-exchange is a closed sub-algebra. We give a formula for the dimension of this sub-algebra as a sum of squares which leads to the understanding of the WA-decomposition of the algebra.

Section 8 gives a summary of our results and outlines interesting future directions for research. Among those directions, we mention a new type of statistical models based on Young diagrams, the quest for holographic duals of tensor models and an intriguing connection between Computational Complexity Theory and correlators in matrix and tensor models.

In the last part of the paper, we have four appendices: appendix A gathers basics of representation theory of the symmetric group $S_{n}$ which is used thoroughly in the text. Appendix B consists in proofs of statements about PCAs, properties of their bases and their centre. Appendix $\mathrm{C}$ provides an illustration of $\mathcal{K}(n=3)$ as a graph algebra and gives its multiplication table. Finally, in appendix D, we give a summary of the calculation of Gaussian correlators (one-point and two-point functions). 
Note added. While this paper was being completed, a few papers with some overlap [38, $58,59]$ appeared. We will be pointing out the specific overlaps in key points as they arise, particularly in section 5. Representation theory and Young diagram combinatorics have also been employed in an SYK context in [60].

\section{Observables in tensor models using permutations}

We start by giving a summary of the description of tensor model observables in terms of permutations which was introduced in [41].

Consider $\left\{V_{i}\right\}_{i=1, \cdots, d}$, a family of complex vector spaces of respective dimensions $N_{1}$, $N_{2}, \ldots, N_{d}$. Let $\Phi$ be a rank $d \geq 2$ covariant tensor with components $\Phi_{i_{1}, \cdots, i_{d}}$, with $i_{a} \in\left\{1, \ldots, N_{a}\right\}, a=1,2, \ldots, d$, transforming as $\otimes_{a=1}^{d} V_{a}$. No symmetry is assumed under permutation of the indices of $\Phi_{i_{1}, \cdots, i_{d}}$. The tensor $\Phi$ transforms under the action of the tensor product of fundamental representations of unitary groups $\otimes_{a=1}^{d} \mathrm{U}\left(N_{a}\right)$ where each $\mathrm{U}\left(N_{a}\right)$ independently acts on a tensor index $i_{a}$. The complex conjugate $\bar{\Phi}_{i_{1} i_{2} \ldots i_{d}}$ of $\Phi_{i_{1} i_{2} \ldots i_{d}}$ is a contravariant tensor of the same rank $d$. The following transformation rule holds:

$$
\begin{aligned}
\Phi_{i_{1} i_{2} \ldots i_{d}} & =\sum_{j_{1}, \ldots, j_{d}} U_{i_{1} j_{1}}^{(1)} U_{i_{2} j_{2}}^{(2)} \ldots U_{i_{d} j_{d}}^{(d)} \Phi_{j_{1} j_{2} \ldots j_{d}} \\
\bar{\Phi}_{i_{1} i_{2} \ldots i_{d}} & =\sum_{j_{1}, \ldots, j_{d}} \bar{U}_{i_{1} j_{1}}^{(1)} \bar{U}_{i_{2} j_{2}}^{(2)} \ldots \bar{U}_{i_{d} j_{d}}^{(d)} \bar{\Phi}_{j_{1} j_{2} \ldots j_{d}}
\end{aligned}
$$

where $U^{(a)}$ are unitary belonging to $\mathrm{U}\left(N_{a}\right), a=1,2, \ldots, d$ and may be all distinct. The rank $d=2$ will be referred to as matrix case and will be useful to make contact with known results in matrix models. We will however focus in the rank $d \geq 3$.

Unitary invariants with respect to the action (2.1) are built by contracting pairs of indices of (covariant and contravariant) tensors. These contractions are in bijection with regular bipartite $d$-colored graphs (see section 2.1 in [41], for illustrations).

The unitary invariants are called observables of tensor models. Take $n$ covariant tensor fields $\Phi$ and $n$ contravariant tensor fields $\bar{\Phi}$. Invariants of the unitary group action built from these are polynomial functions of the tensor variables which we will refer to as tensor invariants of degree $n$. The observables are constructed by contracting indices from the $n$ copies of $\Phi$ and the $n$ copies of $\bar{\Phi}$. The different contractions are labelled by $d$ permutations, $\sigma_{1}, \sigma_{2}, \ldots, \sigma_{d} \in S_{n}$ and the corresponding observables are denoted $\mathcal{O}_{\sigma_{1}, \sigma_{2}, \cdots, \sigma_{d}}(\Phi, \bar{\Phi})$ (see figure 1). There is an equivalence under right and left diagonal action of $S_{n}$ on $S_{n}^{\times d}$ as [41]:

$$
\left(\sigma_{1}, \sigma_{2}, \cdots, \sigma_{d}\right) \sim\left(\mu_{1} \sigma_{1} \mu_{2}, \mu_{1} \sigma_{2} \mu_{2}, \cdots, \mu_{1} \sigma_{d} \mu_{2}\right)
$$

where $\mu_{1,2} \in S_{n}$. Equivalent permutation tuples give rise to the same observable. Thus, counting observables is counting points in the double coset

$$
\operatorname{Diag}\left(S_{n}\right) \backslash\left(S_{n} \times S_{n} \times \cdots \times S_{n}\right) / \operatorname{Diag}\left(S_{n}\right)
$$




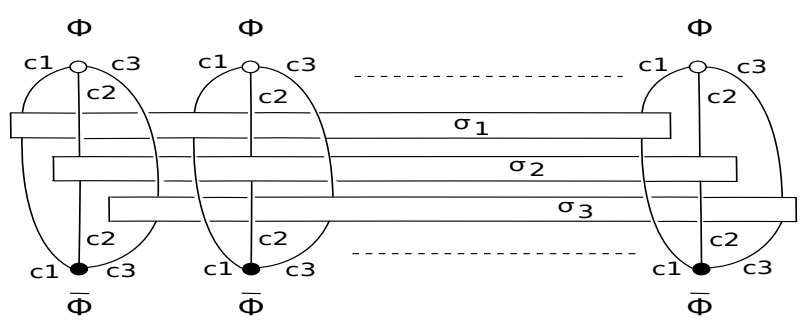

Figure 1. Diagrammatic rank-3 tensor contraction defining $\left(\sigma_{1}, \sigma_{2}, \sigma_{3}\right)$.

We denote the number of points in this double coset as $Z_{d}(n)$. Using the Burnside lemma, we obtain the counting [41]:

$$
Z_{d}(n)=\frac{1}{(n !)^{2}} \sum_{\mu_{i} \in S_{n}} \sum_{\sigma_{i} \in S_{n}} \prod_{i=1}^{d} \delta\left(\mu_{1} \sigma_{i} \mu_{2} \sigma_{i}^{-1}\right)
$$

which can be simplified to

$$
Z_{d}(n)=\sum_{p \vdash n}(\operatorname{Sym}(p))^{d-2}, \quad \operatorname{Sym}(p):=\prod_{i=1}^{n}\left(i^{p_{i}}\right)\left(p_{i} !\right)
$$

where the sum is performed over partitions of $n$ specified by $p=\left\{p_{1}, p_{2}, \cdots, p_{n}\right\}$ where the partition has $p_{1}$ copies of $1, p_{2}$ copies of 2 etc. so that $n=\sum_{i} i p_{i}$. A partition of $p$ of $n$, denoted $p \vdash n$, specifies a cycle structure of permutations $\sigma \in S_{n}$. A cycle structure corresponds to a conjugacy class in $S_{n}$. The conjugacy class corresponding to $p$ will be denoted $T_{p}$. A permutation in $T_{p}$ has a symmetry factor, which is the number of $S_{n}$ permutations leaving it unchanged under conjugation. This is denoted as $\operatorname{Sym}(\sigma)$ and is the same for any permutation $\sigma \in T_{p}$, so we also denote this number as $\operatorname{Sym}(p)$.

An important feature of this paper is that we will extend the correspondence between permutations in $S_{n}^{\times d}$ and observables

$$
\left(\sigma_{1}, \cdots, \sigma_{d}\right) \rightarrow \mathcal{O}_{\sigma_{1}, \cdots, \sigma_{d}}(\Phi, \bar{\Phi})
$$

to the group algebra $\left(\mathbb{C}\left(S_{n}\right)\right)^{\otimes d}$ by linearity

$$
\sum_{\sigma_{1}, \cdots, \sigma_{d}} \lambda^{\sigma_{1}, \cdots, \sigma_{d}}\left(\sigma_{1} \otimes \cdots \otimes \sigma_{d}\right) \rightarrow \sum_{\sigma_{1}, \cdots, \sigma_{d}} \lambda^{\sigma_{1}, \cdots, \sigma_{d}} \mathcal{O}_{\sigma_{1}, \cdots, \sigma_{d}}(\Phi, \bar{\Phi})
$$

We will consider sub-algebras of $\left(\mathbb{C}\left(S_{n}\right)\right)^{\otimes d}$ defined by these equivalences (2.2). We will describe special bases in these sub-algebras constructed using representation theory, which reveal the matrix structure of these sub-algebras expected from the WA theorem.

We now introduce the second fundamental ingredient for our analysis, the measure over the complex tensors

$$
d \mu(\Phi, \bar{\Phi}) \equiv \prod_{i_{k}} d \Phi_{i_{1} i_{2} \ldots i_{d}} d \bar{\Phi}_{i_{1} i_{2} \ldots i_{d}} e^{-\sum_{i_{k}} \Phi_{i_{1} i_{2} \ldots i_{d}} \bar{\Phi}_{i_{1} i_{2} \ldots i_{d}}}
$$


which defines the Gaussian tensor models of interest. Expectation values of observables $\mathcal{O}_{\sigma_{1}, \sigma_{2}, \cdots, \sigma_{d}}(\Phi, \bar{\Phi})$ (which we will denote as $\mathcal{O}_{\sigma_{1}, \sigma_{2}, \cdots, \sigma_{d}}$ for brevity) are defined as

$$
\left\langle\mathcal{O}_{\sigma_{1}, \sigma_{2}, \cdots, \sigma_{d}}\right\rangle=\frac{\int d \mu(\Phi, \bar{\Phi}) \mathcal{O}_{\sigma_{1}, \sigma_{2}, \cdots, \sigma_{d}}}{\int d \mu(\Phi, \bar{\Phi})}
$$

These correlators can be evaluated by summing over Wick contractions, which can be parametrized by permutations $\gamma \in S_{n}$. Thus a graph configuration in the expansion of $\left\langle\mathcal{O}_{\sigma_{1}, \sigma_{2}, \cdots, \sigma_{d}}\right\rangle$, is determined by $\left(\gamma, \sigma_{1}, \sigma_{2}, \cdots, \sigma_{d}\right)$. Summing over all $\gamma$ 's gives the full correlator. Fixing $N_{a}=N$, for all $a=1, \ldots, d$, after some algebra one gets (see appendix D for a derivation of the following equality)

$$
\left\langle\mathcal{O}_{\sigma_{1}, \sigma_{2}, \cdots, \sigma_{d}}\right\rangle=\sum_{\gamma \in S_{n}} N^{\mathbf{c}\left(\gamma \sigma_{1}\right)+\mathbf{c}\left(\gamma \sigma_{2}\right)+\cdots+\mathbf{c}\left(\gamma \sigma_{d}\right)}
$$

where $\mathbf{c}(\alpha)$ is the number of cycles of $\alpha$. This formula reflects the fact that correlators depend on the total number of cycles of compositions of the permutations $\gamma \sigma_{i}, i=1, \ldots, d$. The formula (2.10) has also been obtained in [58]. Another natural type of correlator considered in [41] is the insertion of a product of observables in the integral, with the prescription that we do not allow Wick contractions within the observable. These are referred to as "normal ordered" correlators. They are discussed further in the appendix D and section 5 .

\section{The permutation centralizer algebra $\mathcal{K}(n)$}

In this section we will show that the equivalence classes of permutations which define tensor invariants of degree $n$ in the rank $d=3$ case form an associative algebra $\mathcal{K}(n)$. The structure of the algebra $\mathcal{K}(n)$ is intimately related to Kronecker coefficients. Its dimension is equal to the sum of squares of Kronecker coefficients. For higher $d$, we have analogous algebras $\mathcal{K}(n, d)$ with dimensions equal to a sum of squares which can be expressed in terms of higher order products of Kronecker coefficients. This is shown below in section 3.1. In subsequent subsections, we will primarily focus the analysis to the rank $d=3$ case and the algebra $\mathcal{K}(n)$.

\subsection{Counting observables and Kronecker coefficients}

Consider the counting of tensor invariants of degree $n$. We rewrite the above counting (2.5) as

$$
Z_{3}(n)=\sum_{p \vdash n} \operatorname{Sym}(p)=\sum_{p \vdash n} \frac{\operatorname{Sym}(p)}{n !} \sum_{\sigma \in T_{p}} \operatorname{Sym}(p)
$$

For each partition $p$ of $n$, we are summing over all the permutations in that conjugacy class $\sigma \in T_{p}$, and dividing by the size of the conjugacy class $T_{p}$, denoted $\left|T_{p}\right|$. We use the fact that

$$
\left|T_{p}\right|=\frac{n !}{\operatorname{Sym}(p)}
$$


Using identities in appendix A.1, we write

$$
\begin{aligned}
Z_{3}(n) & =\sum_{p \vdash n} \frac{\operatorname{Sym}(p)}{n !} \sum_{\sigma \in T_{p}} \operatorname{Sym}(p)=\frac{1}{n !} \sum_{\sigma \in S_{n}} \operatorname{Sym}(\sigma) \operatorname{Sym}(\sigma) \\
& =\frac{1}{n !} \sum_{\sigma \in S_{n}} \sum_{\gamma_{1}, \gamma_{2} \in S_{n}} \delta\left(\gamma_{1} \sigma \gamma_{1}^{-1} \sigma^{-1}\right) \delta\left(\gamma_{2} \sigma \gamma_{2}^{-1} \sigma^{-1}\right) \\
& =\frac{1}{n !} \sum_{\sigma \in S_{n}} \sum_{R_{1}, R_{2} \vdash n} \chi^{R_{1}}(\sigma) \chi^{R_{1}}(\sigma) \chi^{R_{2}}(\sigma) \chi^{R_{2}}(\sigma) \\
& =\frac{1}{(n !)^{2}} \sum_{\gamma \in S_{n}} \sum_{\sigma_{l} \in S_{n}} \sum_{R_{1}, R_{2} \vdash n} \chi^{R_{1}}\left(\sigma_{1}\right) \chi^{R_{2}}\left(\sigma_{1}\right) \delta\left(\sigma_{1} \gamma \sigma_{2} \gamma^{-1}\right) \chi^{R_{1}}\left(\sigma_{2}\right) \chi^{R_{2}}\left(\sigma_{2}\right) \\
& =\frac{1}{(n !)^{2}} \sum_{\sigma_{l} \in S_{n}} \sum_{R_{1}, R_{2} \vdash n} \chi^{R_{1}}\left(\sigma_{1}\right) \chi^{R_{2}}\left(\sigma_{1}\right)\left(\sum_{S \vdash n} \chi^{S}\left(\sigma_{1}\right) \chi^{S}\left(\sigma_{2}\right)\right) \chi^{R_{1}}\left(\sigma_{2}\right) \chi^{R_{2}}\left(\sigma_{2}\right) \\
& =\sum_{R_{1}, R_{2}, S \vdash n}\left(\mathrm{C}\left(R_{1}, R_{2}, S\right)\right)^{2}
\end{aligned}
$$

where the symbol

$$
\mathrm{C}\left(R_{1}, R_{2}, R_{3}\right)=\frac{1}{n !} \sum_{\sigma \in S_{n}} \chi^{R_{1}}(\sigma) \chi^{R_{2}}(\sigma) \chi^{R_{3}}(\sigma)
$$

is the Kronecker coefficient or multiplicity of the irreducible representation (irrep) $R_{3}$ in the tensor product of the irreps $R_{1}$ and $R_{2}$. Equivalently it is the multiplicity of the onedimensional representation in the tensor product $R_{1} \otimes R_{2} \otimes R_{3}$. Similar manipulations show that the same counting is also equal to

$$
Z_{3}(n)=\sum_{R_{1}, R_{2}, S \vdash n} \mathrm{C}\left(R_{1}, R_{1}, S\right) \mathrm{C}\left(R_{2}, R_{2}, S\right)
$$

Hence, counting observables of tensor model of rank 3 coincides with a sum of squares (or product) of Kronecker coefficients. That sum is also the dimension of an algebra $\mathcal{K}(n)$ that we will discuss in the next section. The connection between the counting of tensors and Kronecker coefficients has also been discussed in the physics literature in [38, 52, 59] and in the mathematics literature in [61]. For future reference in this paper, a key point from the above discussion is

$$
\operatorname{dim}(\mathcal{K}(n))=\sum_{R_{1}, R_{2}, S \vdash n}\left(\mathrm{C}\left(R_{1}, R_{2}, S\right)\right)^{2}
$$

Counting in rank- $\boldsymbol{d}$ tensors. The above counting generalizes quite naturally at any rank as

$$
\begin{aligned}
Z_{d}(n) & =\sum_{p \vdash n} \frac{\operatorname{Sym}(p)^{d-2}}{n !} \sum_{\sigma \in T_{p}} \operatorname{Sym}(p)=\frac{1}{n !} \sum_{\sigma \in S_{n}} \operatorname{Sym}(\sigma)^{d-1} \\
& =\frac{1}{n !} \sum_{\sigma \in S_{n}} \sum_{\gamma_{1}, \gamma_{2}, \ldots, \gamma_{d} \in S_{n}} \delta\left(\gamma_{1} \sigma \gamma_{1}^{-1} \sigma^{-1}\right) \delta\left(\gamma_{2} \sigma \gamma_{2}^{-1} \sigma^{-1}\right) \ldots \delta\left(\gamma_{d-1} \sigma \gamma_{d-1}^{-1} \sigma^{-1}\right) \\
& =\frac{1}{n !} \sum_{\sigma \in S_{n}} \prod_{i=1}^{d-1}\left[\sum_{R_{i} \vdash n} \chi^{R_{i}}(\sigma) \chi^{R_{i}}(\sigma)\right]
\end{aligned}
$$


where we used (A.21) of appendix A.1. Then we re-introduce delta-functions which couple different permutations as:

$$
\begin{aligned}
Z_{d}(n)= & \frac{1}{(n !)^{2}} \sum_{\gamma \in S_{n}} \sum_{\sigma_{l} \in S_{n}} \sum_{R_{1}, R_{2} \vdash n} \chi^{R_{1}}\left(\sigma_{1}\right) \chi^{R_{1}}\left(\sigma_{1}\right) \delta\left(\sigma_{1} \gamma \sigma_{2} \gamma^{-1}\right) \chi^{R_{2}}\left(\sigma_{2}\right) \chi^{R_{2}}\left(\sigma_{2}\right) \\
& \times \prod_{i=3}^{d-1}\left[\sum_{R_{i} \vdash n} \chi^{R_{i}}\left(\sigma_{2}\right) \chi^{R_{i}}\left(\sigma_{2}\right)\right] \\
= & \frac{1}{(n !)^{3}} \sum_{\sigma_{l} \in S_{n}} \sum_{R_{1}, R_{2} \vdash n} \chi^{R_{1}}\left(\sigma_{1}\right) \chi^{R_{1}}\left(\sigma_{1}\right)\left(\sum_{S_{1} \vdash n} \chi^{S_{1}}\left(\sigma_{1}\right) \chi^{S_{1}}\left(\sigma_{2}\right)\right) \chi^{R_{2}}\left(\sigma_{2}\right) \chi^{R_{2}}\left(\sigma_{2}\right) \\
& \times\left[\sum_{S_{2} \vdash n} \chi^{S_{2}}\left(\sigma_{2}\right) \chi^{S_{2}}\left(\sigma_{3}\right)\right] \chi^{R_{3}}\left(\sigma_{3}\right) \chi^{R_{3}}\left(\sigma_{3}\right) \prod_{i=4}^{d-1}\left[\sum_{R_{i} \vdash n} \chi^{R_{i}}\left(\sigma_{3}\right) \chi^{R_{i}}\left(\sigma_{3}\right)\right] \\
= & \sum_{R_{i}, S_{i} \vdash n} \mathrm{C}\left(R_{1}, R_{1}, S_{1}\right) \mathrm{C}_{4}\left(R_{2}, R_{2}, S_{1}, S_{2}\right) \mathrm{C}_{4}\left(R_{3}, R_{3}, S_{2}, S_{3}\right) \ldots \\
& \times \mathrm{C}_{4}\left(R_{d-2}, R_{d-2}, S_{d-3}, S_{d-2}\right) \mathrm{C}\left(R_{d-1}, R_{d-1}, S_{d-2}\right) \\
= & \sum_{R_{i}, S_{i} \vdash n} \mathrm{C}\left(R_{1}, R_{1}, S_{1}\right)\left[\prod_{i=2}^{d-2} \mathrm{C}_{4}\left(R_{i}, R_{i}, S_{i-1}, S_{i}\right)\right] \mathrm{C}\left(R_{d-1}, R_{d-1}, S_{d-2}\right)
\end{aligned}
$$

where the symbol $\mathrm{C}_{4}(\cdot)$ stands for

$$
\begin{aligned}
\mathrm{C}_{4}\left(R_{1}, R_{2}, R_{3}, R_{4}\right) & =\frac{1}{n !} \sum_{\sigma \in S_{n}} \chi^{R_{1}}(\sigma) \chi^{R_{2}}(\sigma) \chi^{R_{3}}(\sigma) \chi^{R_{4}}(\sigma) \\
& =\frac{1}{(n !)^{2}} \sum_{\gamma, \sigma_{1}, \sigma_{2} \in S_{n}} \chi^{R_{1}}\left(\sigma_{1}\right) \chi^{R_{2}}\left(\sigma_{1}\right)\left(\delta\left(\sigma_{1} \gamma \sigma_{2} \gamma^{-1}\right)\right) \chi^{R_{3}}\left(\sigma_{2}\right) \chi^{R_{4}}\left(\sigma_{2}\right) \\
& =\frac{1}{(n !)^{2}} \sum_{\sigma_{1}, \sigma_{2} \in S_{n}} \chi^{R_{1}}\left(\sigma_{1}\right) \chi^{R_{2}}\left(\sigma_{1}\right)\left(\sum_{S \vdash n} \chi^{S}\left(\sigma_{1}\right) \chi^{S}\left(\sigma_{2}\right)\right) \chi^{R_{3}}\left(\sigma_{2}\right) \chi^{R_{4}}\left(\sigma_{2}\right) \\
& =\sum_{S \vdash n} \mathrm{C}\left(R_{1}, R_{2}, S\right) \mathrm{C}\left(S, R_{3}, R_{4}\right)
\end{aligned}
$$

Thus at any rank the counting of observables of tensor models maps to a sum of products of Kronecker coefficients. For example, at rank $d=4$, we obtain

$$
Z_{d}(4)=\sum_{R_{i}, S_{i} \vdash n} \mathrm{C}\left(S_{2}, S_{1}, S_{3}\right) \mathrm{C}\left(R_{1}, R_{1}, S_{1}\right) \mathrm{C}\left(R_{2}, R_{2}, S_{2}\right) \mathrm{C}\left(R_{3}, R_{3}, S_{3}\right)
$$

To write a compact formula as a sum of squares, we introduce

$$
\mathrm{C}_{k}\left(R_{1}, R_{2}, \cdots, R_{k}\right)=\frac{1}{n !} \sum_{\sigma \in S_{n}} \chi^{R_{1}}(\sigma) \chi^{R_{2}}(\sigma) \cdots \chi^{R_{k}}(\sigma)
$$

This counts the multiplicity of the one-dimensional $S_{n}$ irrep in the tensor product of irreps $R_{1} \otimes \cdots \otimes R_{k}$. It can be expressed in terms of products of Kronecker coefficients. The dimension of $\mathcal{K}(n, d)$ is

$$
Z_{d}(n)=\operatorname{dim}(\mathcal{K}(n, d))=\sum_{R_{1}, \cdots, R_{d-1}, S \vdash n}\left(C_{d}\left(R_{1}, R_{2}, \cdots, R_{d-1}, S\right)\right)^{2}
$$




\section{$3.2 \mathcal{K}(n)$ as a centralizer algebra in $\mathbb{C}\left(S_{n}\right) \otimes \mathbb{C}\left(S_{n}\right)$}

The permutation equivalence classes in $S_{n} \times S_{n} \times S_{n}$ described earlier (2.2) have a gaugefixed formulation involving pairs of permutations. One way to see this [41] is by manipulating the symmetric group delta functions which implement the Burnside lemma counting. For example, we can choose $\mu_{1}=\sigma_{1}^{-1}$ which maps the triple

$$
\left(\sigma_{1}, \sigma_{2}, \sigma_{3}\right) \rightarrow\left(1, \sigma_{1}^{-1} \sigma_{2}, \sigma_{1}^{-1} \sigma_{3}\right) \equiv\left(1, \tau_{1}, \tau_{2}\right)
$$

The $\mu_{2}=\mu$ equivalence now acts on $\left(\tau_{1}, \tau_{2}\right)$ as

$$
\left(\tau_{1}, \tau_{2}\right) \sim\left(\mu \tau_{1} \mu^{-1}, \mu \tau_{2} \mu^{-1}\right)
$$

We will therefore define $\mathcal{K}(n)$ as the sub-algebra of group algebra $\mathbb{C}\left(S_{n}\right) \otimes \mathbb{C}\left(S_{n}\right)$ which is invariant under conjugation by the diagonally embedded $S_{n}$. In this section, we detail the structure of this sub-algebra.

Consider the elements of $\mathbb{C}\left(S_{n}\right) \otimes \mathbb{C}\left(S_{n}\right)$ obtained by starting with a tensor product $\sigma_{1} \otimes \sigma_{2}$ and summing all their conjugates by $\gamma$ acting diagonally

$$
\sigma_{1} \otimes \sigma_{2} \rightarrow \sum_{\gamma \in S_{n}} \gamma \sigma_{1} \gamma^{-1} \otimes \gamma \sigma_{2} \gamma^{-1}
$$

Now, $\mathcal{K}(n) \subset \mathbb{C}\left(S_{n}\right) \otimes \mathbb{C}\left(S_{n}\right)$ is the vector space over $\mathbb{C}$ spanned by all $\sum_{\gamma \in S_{n}} \gamma \sigma_{1} \gamma^{-1} \otimes$ $\gamma \sigma_{2} \gamma^{-1}, \sigma_{1}$ and $\sigma_{2} \in S_{n}$ :

$$
\mathcal{K}(n)=\operatorname{Span}_{\mathbb{C}}\left\{\sum_{\gamma \in S_{n}} \gamma \sigma_{1} \gamma^{-1} \otimes \gamma \sigma_{2} \gamma^{-1}, \sigma_{1}, \sigma_{2} \in S_{n}\right\}
$$

By construction, the elements of $\mathcal{K}(n)$ are invariants under the action of $\operatorname{Diag}\left(\mathbb{C}\left(S_{n}\right)\right)$. To verify this, we evaluate any element $A \in \mathcal{K}(n)$

$$
(\tau \otimes \tau) \cdot A \cdot\left(\tau^{-1} \otimes \tau^{-1}\right)=\sum_{\sigma_{1}, \sigma_{2}, \gamma \in S_{n}} c_{\sigma_{1}, \sigma_{2}} \tau \gamma \sigma_{1} \gamma^{-1} \tau^{-1} \otimes \tau \gamma \sigma_{2} \gamma^{-1} \tau^{-1}=A
$$

where we redefine $\gamma \rightarrow \tau \gamma$.

Proposition 1. $\mathcal{K}(n)$ is an associative unital sub-algebra of $\mathbb{C}\left(S_{n}\right) \otimes \mathbb{C}\left(S_{n}\right)$.

Proof. We verify that $\mathcal{K}(n)$ is a closed under multiplication. Take two elements of $\mathcal{K}(n)$, $A=\sum_{\sigma_{1}, \sigma_{2}, \gamma_{1} \in S_{n}} c_{\sigma_{1}, \sigma_{2}} \gamma_{1} \sigma_{1} \gamma_{1}^{-1} \otimes \gamma_{1} \sigma_{2} \gamma_{1}^{-1}$ and $B=\sum_{\tau_{1}, \tau_{2}, \gamma_{2} \in S_{n}} c_{\tau_{1}, \tau_{2}}^{\prime} \gamma_{2} \tau_{1} \gamma_{2}^{-1} \otimes \gamma_{2} \tau_{2} \gamma_{2}^{-1}$, with coefficients $c_{\sigma_{1}, \sigma_{2}}$ and $c_{\tau_{1}, \tau_{2}}^{\prime}$,

$$
\begin{aligned}
A B & =\sum_{\sigma_{i}, \tau_{i} \in S_{n}} c_{\sigma_{1}, \sigma_{2}} c_{\tau_{1}, \tau_{2}}^{\prime} \sum_{\gamma_{1}, \gamma_{2}} \gamma_{1} \sigma_{1} \gamma_{1}^{-1} \gamma_{2} \tau_{1} \gamma_{2}^{-1} \otimes \gamma_{1} \sigma_{2} \gamma_{1}^{-1} \gamma_{2} \tau_{2} \gamma_{2}^{-1} \\
& =\sum_{\gamma} \sum_{\sigma_{i}, \tau_{i} \in S_{n}} c_{\sigma_{1}, \sigma_{2}} c_{\tau_{1}, \tau_{2}}^{\prime} \sum_{\gamma_{1}} \gamma_{1}\left(\sigma_{1} \gamma \tau_{1} \gamma^{-1}\right) \gamma_{1}^{-1} \otimes \gamma_{1}\left(\sigma_{2} \gamma \tau_{2} \gamma^{-1}\right) \gamma_{1}^{-1}
\end{aligned}
$$

where we redefined $\gamma=\gamma_{1}^{-1} \gamma_{2}, \gamma_{2}^{-1}=\gamma^{-1} \gamma_{1}^{-1}$. Clearly, the last line shows that $A B$ belongs to $\mathcal{K}(n)$ as a linear combination of basis elements.

The unit of $\mathbb{C}\left(S_{n}\right)^{\otimes 2}$ is $i d \otimes i d$ which also belongs to $\mathcal{K}(n)$. One can also check that $\mathcal{K}(n)$ is an associative algebra because $\mathbb{C}\left(S_{n}\right)$ is associative. Hence $\mathcal{K}(n)$ is a sub-algebra of $\mathbb{C}\left(S_{n}\right) \otimes \mathbb{C}\left(S_{n}\right)$. 
The dimension of $\mathcal{K}(n)$ is associated with the number of observables of tensor models. Indeed, each colored tensor graph is associated with an equivalence relation (3.15) also associated with one basis element of $\mathcal{K}(n)$. Thus $\operatorname{dim}_{\mathbb{C}} \mathcal{K}(n)=Z_{3}(n)$. In the following, the elements of $\mathcal{K}(n)$ are then called as and identified with "graphs" and the basis (3.16) will be called graph-basis.

A Fourier basis of invariants. The Fourier transform of the basis (3.15) of $\mathcal{K}(n)$ determines another basis of invariants for $\mathcal{K}(n)$. The elements of this basis are labelled by $\left(R, S, T, \tau_{1}, \tau_{2}\right)$ and are of the form

$$
Q_{\tau_{1}, \tau_{2}}^{R, S, T}=\kappa_{R, S} \sum_{\sigma_{1}, \sigma_{2} \in S_{n}} \sum_{i_{1}, i_{2}, i_{3}, j_{1}, j_{2}} C_{i_{1}, i_{2} ; i_{3}}^{R, S ; T, \tau_{1}} C_{j_{1}, j_{2} ; i_{3}}^{R, S ; T, \tau_{2}} D_{i_{1} j_{1}}^{R}\left(\sigma_{1}\right) D_{i_{2} j_{2}}^{S}\left(\sigma_{2}\right) \sigma_{1} \otimes \sigma_{2}
$$

where $\kappa_{R, S}=\frac{d(R) d(S)}{(n !)^{2}}, i_{1}$ and $j_{1}$ (resp. $i_{2}$ and $j_{2}$ ) are positive integers bounded by the dimension $d(R)$ (resp. $d(S)$ ) of the representation of $S_{n}$, and $i_{3}$ by $d(T)$. Meanwhile, $C_{i_{1}, i_{2} ; i_{3}}^{R, S, \tau_{1}}$ are Clebsch-Gordan coefficients involved in the tensor products of representations of $S_{n}$, see appendix A.2 for a brief definition and properties that we will use hereafter; the multiplicities $\tau_{1}$ and $\tau_{2} \in \llbracket 1, \mathrm{C}(R, S, T) \rrbracket$. We can check that, by acting by the diagonal action, the basis elements are invariant (for the proof see (B.1) in appendix B.1):

$$
(\gamma \otimes \gamma) \cdot Q_{\tau_{1}, \tau_{2}}^{R, S T} \cdot\left(\gamma^{-1} \otimes \gamma^{-1}\right)=Q_{\tau_{1}, \tau_{2}}^{R, S}
$$

The basis $\left\{Q_{\tau_{1}, \tau_{2}}^{R, S}\right\}$, shortly called $Q$-basis in the following, makes explicit that the dimension of the algebra $\mathcal{K}(n)$ is given by

$$
\sum_{R, S, T} \mathrm{C}(R, S, T)^{2}
$$

An important property of the $Q_{\tau_{1}, \tau_{2}}^{R, S,}$ 's is that they are matrix bases of $\mathcal{K}(n)$. We have (the proof of the following is detailed in (B.3) of appendix B.1):

$$
Q_{\tau_{1}, \tau_{2}}^{R, S, T} Q_{\tau_{2}^{\prime}, \tau_{3}}^{R^{\prime}, T^{\prime}}=\delta_{R R^{\prime}} \delta_{S S^{\prime}} \delta_{T T^{\prime}} \delta_{\tau_{2} \tau_{2}^{\prime}} Q_{\tau_{1}, \tau_{3}}^{R, S}
$$

Finally, noting that $\mathrm{C}(R, S, T)$ is at most 1 for $n \leq 4$, then the matrices $Q_{\tau_{1}, \tau_{2}}^{R, S T}$ are $1 \times 1$ hence are commuting. Consequently, at lower order in $n \leq 4, \mathcal{K}(n)$ is commutative.

Orthogonality of the $\boldsymbol{Q}$-basis. Consider the bilinear pairing $\boldsymbol{\delta}_{2}: \mathbb{C}\left(S_{n}\right)^{\otimes d} \times \mathbb{C}\left(S_{n}\right)^{\otimes d} \rightarrow$ $\mathbb{C}, d \geq 0$,

$$
\boldsymbol{\delta}_{d}\left(\otimes_{i=1}^{d} \sigma_{i} ; \otimes_{i=1}^{d} \sigma_{i}^{\prime}\right)=\prod_{i=1}^{d} \delta\left(\sigma_{i} \sigma_{i}^{\prime-1}\right)
$$

which extends to linear combination with complex coefficients naturally:

$$
\boldsymbol{\delta}_{d}\left(\sum_{\sigma_{l}} c_{\left\{\sigma_{l}\right\}} \otimes_{l=1}^{d} \sigma_{l} ; \sum_{\sigma_{l}^{\prime}} c_{\left\{\sigma_{l}^{\prime}\right\}}^{\prime} \otimes_{l=1}^{d} \sigma_{l}^{\prime}\right)=\sum_{\sigma_{l}} c_{\left\{\sigma_{l}\right\}} c_{\left\{\sigma_{l}\right\}}^{\prime}
$$

We can also consider an inner product, i.e. a sesquilinear pairing, where we would have $\bar{c}_{\left\{\sigma_{l}\right\}}$ on the r.h.s. above. The inner product will have the same non-degeneracy property we discuss below for the bilinear form. 
The following proposition can be easily checked.

Proposition 2. $\boldsymbol{\delta}_{d}$ is a non-degenerate pairing on $\mathbb{C}\left(S_{n}\right)^{\otimes d} \forall d \geq 1$.

Inspecting the pairing of basis elements of $\left\{\sum_{\gamma} \gamma \sigma_{1} \gamma^{-1} \otimes \gamma \sigma_{2} \gamma^{-1}\right\}$ in $\mathcal{K}(n)$, we have

$$
\begin{aligned}
\boldsymbol{\delta}_{2}( & \left.\sum_{\gamma_{1}} \gamma_{1} \sigma_{1} \gamma_{1}^{-1} \otimes \gamma_{1} \sigma_{2} \gamma_{1}^{-1} ; \sum_{\gamma_{2}} \gamma_{2} \tau_{1} \gamma_{2}^{-1} \otimes \gamma_{2} \tau_{2} \gamma_{2}^{-1}\right) \\
= & \sum_{\gamma_{1}, \gamma_{2}} \delta\left(\gamma_{1} \sigma_{1} \gamma_{1}^{-1}\left(\gamma_{2} \tau_{1} \gamma_{2}^{-1}\right)^{-1}\right) \delta\left(\gamma_{1} \sigma_{2} \gamma_{1}^{-1}\left(\gamma_{2} \tau_{2} \gamma_{2}^{-1}\right)^{-1}\right) \\
& =\sum_{\gamma_{1}, \gamma_{2}} \delta\left(\gamma_{1} \sigma_{1} \gamma_{1}^{-1} \gamma_{2} \tau_{1}^{-1} \gamma_{2}^{-1}\right) \delta\left(\gamma_{1} \sigma_{2} \gamma_{1}^{-1} \gamma_{2} \tau_{2}^{-1} \gamma_{2}^{-1}\right) \\
& =\sum_{\gamma_{1}, \gamma_{2}} \delta\left(\gamma_{1} \sigma_{1} \gamma_{2} \tau_{1}^{-1}\left(\gamma_{1} \gamma_{2}\right)^{-1}\right) \delta\left(\gamma_{1} \sigma_{2} \gamma_{2} \tau_{2}^{-1}\left(\gamma_{1} \gamma_{2}\right)^{-1}\right) \\
& =n ! \sum_{\gamma} \delta\left(\sigma_{1} \gamma \tau_{1}^{-1} \gamma^{-1}\right) \delta\left(\sigma_{2} \gamma \tau_{2}^{-1} \gamma^{-1}\right)
\end{aligned}
$$

which is not vanishing whenever the $\sigma_{i}$ 's are conjugate to $\tau_{i}$ 's, $i=1,2$. This is precisely saying that the two basis elements and the corresponding graphs are in the same class. The above sum over $\gamma$ computes to the order of the automorphism group of the graph associated with any of the basis element. Consider two colored tensor graphs $G_{\sigma_{1}, \sigma_{2}}$ and $G_{\sigma_{1}^{\prime}, \sigma_{2}^{\prime}}$ associated with the basis elements $\sum_{\gamma} \gamma \sigma_{1} \gamma^{-1} \otimes \gamma \sigma_{2} \gamma^{-1}$ and $\sum_{\gamma} \gamma \sigma_{1}^{\prime} \gamma^{-1} \otimes \gamma \sigma_{2}^{\prime} \gamma^{-1}$, respectively, then we write

$$
\boldsymbol{\delta}_{2}\left(\sum_{\gamma_{1}} \gamma_{1} \sigma_{1} \gamma_{1}^{-1} \otimes \gamma_{1} \sigma_{2} \gamma_{1}^{-1} ; \sum_{\gamma_{2}} \gamma_{2} \tau_{1} \gamma_{2}^{-1} \otimes \gamma_{2} \tau_{2} \gamma_{2}^{-1}\right)=n ! \delta\left(G_{\sigma_{1}, \sigma_{2}}, G_{\sigma_{1}^{\prime}, \sigma_{2}^{\prime}}\right)\left|\operatorname{Aut}\left(G_{\sigma_{1}, \sigma_{2}}\right)\right|
$$

where $\delta\left(G_{\sigma_{1}, \sigma_{2}}, G_{\sigma_{1}^{\prime}, \sigma_{2}^{\prime}}\right)=1$ if the graphs are equivalent and 0 otherwise, and $\left|\operatorname{Aut}\left(G_{\sigma_{1}, \sigma_{2}}\right)\right|$ is the order the automorphism group $\operatorname{Aut}\left(G_{\sigma_{1}, \sigma_{2}}\right)$ of the graph $G_{\sigma_{1}, \sigma_{2}}$. In the end, the restriction of $\boldsymbol{\delta}_{2}$ to $\mathcal{K}(n)$ is non degenerate and the basis of invariants is orthogonal with respect to that product. The following statement is therefore obvious

Proposition 3. $\mathcal{K}(n)$ is an associative unital semi-simple algebra.

Semi-simple algebras and their isomorphism to a direct sum of matrix algebras (WA theorem) are explained in [53, 54].

The $Q$-basis proves to be orthogonal with respect to the bilinear pairing $\boldsymbol{\delta}_{2}$ (see (B.4) of appendix B.1)

$$
\boldsymbol{\delta}_{2}\left(Q_{\tau_{1}, \tau_{1}^{\prime}}^{R, S, T} ; Q_{\tau_{2}, \tau_{2}^{\prime}}^{R^{\prime}, T^{\prime}}\right)=\kappa_{R, S} d(T) \delta_{R R^{\prime}} \delta_{S S^{\prime}} \delta_{T T^{\prime}} \delta_{\tau_{1} \tau_{2}} \delta_{\tau_{1}^{\prime} \tau_{2}^{\prime}}
$$

Note that we could have changed the normalization $\kappa_{R, S}$ of $Q_{\tau_{1}, \tau_{2}}^{R, T}$ to make that basis orthonormal with respect to $\boldsymbol{\delta}_{2}$. However, the previous choice of making as simple as possible the matrix multiplication of the $Q$ 's has fixed the normalization $\kappa_{R S}$. Another option to make the bilinear pairing of $Q$ 's normalized is to change the definition of the pairing itself, but we will keep the present definition of $\boldsymbol{\delta}_{d}$ for simplicity. The orthogonality 
relation (3.27) reveals that the basis $\left\{Q_{\tau, \tau^{\prime}}^{R, T}\right\}$ decomposes $\mathcal{K}(n)$ in orthogonal blocs labelled by $(R, S, T)$ and for each such triple an orthogonal square bloc labelled by $\left(\tau, \tau^{\prime}\right)$.

We can address the expansion of the graph-basis in terms of the $Q$-basis (inverse transform):

$$
\sum_{\gamma} \gamma \sigma_{1} \gamma^{-1} \otimes \gamma \sigma_{2} \gamma^{-1}=\sum_{R, S, T, \tau_{1}, \tau_{2}}\left[\sum_{\gamma} \boldsymbol{\delta}_{2}\left(Q_{\tau_{1}, \tau_{2}}^{R, S, T} ; \gamma \sigma_{1} \gamma^{-1} \otimes \gamma \sigma_{2} \gamma^{-1}\right)\right] Q_{\tau_{1}, \tau_{2}}^{R, T}
$$

where the coefficients calculate as

$$
\begin{aligned}
\sum_{\gamma} \boldsymbol{\delta}_{2}\left(Q_{\tau_{1}, \tau_{2}}^{R, S, T} ; \gamma \sigma_{1} \gamma^{-1} \otimes \gamma \sigma_{2} \gamma^{-1}\right) & =\sum_{\gamma} \boldsymbol{\delta}_{2}\left((\gamma \otimes \gamma) \cdot Q_{\tau_{1}, \tau_{2}}^{R, S, T} \cdot\left(\gamma^{-1} \otimes \gamma^{-1}\right) ; \sigma_{1} \otimes \sigma_{2}\right) \\
=\sum_{\gamma} \boldsymbol{\delta}_{2}\left(Q_{\tau_{1}, \tau_{2}}^{R, S, T} ; \sigma_{1} \otimes \sigma_{2}\right) & =n ! \kappa_{R, S} \sum_{i_{l}, j_{l}} C_{i_{1}, i_{2} ; i_{3}}^{R, S ; T, \tau_{1}} C_{j_{1}, j_{2} ; i_{3}}^{R, S ; T, \tau_{2}} D_{i_{1} j_{1}}^{R}\left(\sigma_{1}\right) D_{i_{2} j_{2}}^{S}\left(\sigma_{2}\right)
\end{aligned}
$$

where used has been made of (3.20), namely the invariance of the $Q$-basis. The coefficient (3.29) can be interpreted as the projection of a graph onto the $Q$-basis.

\subsection{The centre $\mathcal{Z}(\mathcal{K}(n))$ of $\mathcal{K}(n)$}

Using the basis elements $Q_{\tau_{1}, \tau_{2}}^{R, T}$, we build elements of the centre $\mathcal{Z}(\mathcal{K}(n))$ of $\mathcal{K}(n)$ by taking their trace at fixed $(R, S, T)$ :

$$
P^{R, S, T}=\sum_{\tau} Q_{\tau, \tau}^{R, S, T}
$$

To prove that $P^{R, S, T}$ is in the centre $\mathcal{Z}(\mathcal{K}(n))$, it is sufficient to show that it is commuting with the basis elements $Q_{\tau_{1}, \tau_{2}}^{R, S, T}$ of $\mathcal{K}(n)$ :

$$
\begin{aligned}
Q_{\tau_{1}, \tau_{2}}^{R^{\prime}, T^{\prime}, T^{\prime}} \cdot P^{R, S, T} & =\sum_{\tau} Q_{\tau_{1}, \tau_{2}}^{R^{\prime}, S^{\prime}, T^{\prime}} Q_{\tau, \tau}^{R, S, T}=\sum_{\tau} \delta_{R R^{\prime}} \delta_{S S^{\prime}} \delta_{T T^{\prime}} \delta_{\tau_{2} \tau} Q_{\tau_{1}, \tau}^{R, S, T}=\delta_{R R^{\prime}} \delta_{S S^{\prime}} \delta_{T T^{\prime}} Q_{\tau_{1}, \tau_{2}}^{R, S, T} \\
& =\sum_{\tau} \delta_{R R^{\prime}} \delta_{S S^{\prime}} \delta_{T T^{\prime}} \delta_{\tau \tau_{1}} Q_{\tau, \tau_{2}}^{R^{\prime}, S^{\prime}, T^{\prime}}=P^{R, S, T} \cdot Q_{\tau_{1}, \tau_{2}}^{R^{\prime}, S^{\prime}, T^{\prime}}
\end{aligned}
$$

The orthogonality of the $P$ 's follows from the orthogonality of the $Q$-basis (3.27):

$$
\begin{aligned}
\boldsymbol{\delta}_{2}\left(P^{R, S, T} ; P^{R^{\prime}, S^{\prime}, T^{\prime}}\right) & =\sum_{\tau, \tau^{\prime}} \boldsymbol{\delta}_{2}\left(Q_{\tau, \tau}^{R, S, T} ; Q_{\tau^{\prime}, \tau^{\prime}}^{R^{\prime}, S^{\prime}, T^{\prime}}\right) \\
& =\kappa_{R, S} d(T) \delta_{R R^{\prime}} \delta_{S S^{\prime}} \delta_{T T^{\prime}} \sum_{\tau, \tau^{\prime}} \delta_{\tau \tau^{\prime}}=\kappa_{R, S} d(T) \mathrm{C}(R, S, T) \delta_{R R^{\prime}} \delta_{S S^{\prime}} \delta_{T T^{\prime}}
\end{aligned}
$$

Proposition 4. The set $\left\{P^{R, S, T}\right\}$ is a basis of $\mathcal{Z}(\mathcal{K}(n))$ and

$$
\operatorname{dim} \mathcal{Z}(\mathcal{K}(n))=\text { number of non vanishing Kronecker coefficients }
$$

Proof. $\mathcal{K}(n)$ decomposes in irreducible blocs labelled by $(R, S, T)$ and, associated with each of the triples, a matrix $Q_{\tau, \tau^{\prime}}^{R, S}$. In that vector space, for a given $(R, S, T), P^{R, S, T}$ is the sum of diagonal elements of $Q_{\tau, \tau^{\prime}}^{R, S, T}$. Collecting all possible diagonals hence $P^{R, S, T}$ spans the centre $\mathcal{Z}(\mathcal{K}(n))$.

The dimension of $\mathcal{Z}(\mathcal{K}(n))$ is given by the number of non vanishing Kronecker coefficients: a triple $(R, S, T)$, such that $\mathrm{C}(R, S, T) \neq 0$ yields a non vanishing $Q_{\tau, \tau^{\prime}}^{R, T}$ and contributes to a single $P^{R, S, T}$. The result on the dimension of $\mathcal{Z}(\mathcal{K}(n))$ follows. 
An overcomplete basis of central elements. Here we will show how we can start with a triple of irreps of $\mathbb{C}\left(S_{n}\right)$ and construct central elements of $\mathcal{K}(n)$ from them. These will form an overcomplete basis of central elements, which will be demonstated by taking the pairing with the basis $P^{R, S, T}$ described above.

First consider a partition $R$ of $n$ and the element $z_{R}=\sum_{\sigma} \chi^{R}(\sigma) \sigma$ that is central in $\mathbb{C}\left(S_{n}\right)$. Indeed for any $R \vdash n$, choose $\gamma \in S_{n}$ any arbitrary basis element of $\mathbb{C}\left(S_{n}\right)$, and calculate:

$$
\gamma z_{R} \gamma^{-1}=\sum_{\sigma} \chi^{R}(\sigma) \gamma \sigma \gamma^{-1}=\sum_{\sigma} \chi^{R}\left(\gamma \sigma \gamma^{-1}\right) \sigma=z_{R}
$$

Let $R_{1}$ and $R_{2}$ be two partitions of $n$ from which we introduce the central elements $z_{R_{i}}=\sum_{\sigma} \chi^{R_{i}}(\sigma) \sigma, i=1,2$, then build

$$
z_{R_{1}, R_{2}}=z_{R_{1}} \otimes z_{R_{2}}=\sum_{\sigma_{1}, \sigma_{2} \in S_{n}} \chi^{R_{1}}\left(\sigma_{1}\right) \chi^{R_{2}}\left(\sigma_{2}\right) \sigma_{1} \otimes \sigma_{2}
$$

that one can show to be central because is a tensor product of central elements (use (3.34) twice on each sector).

Another possible element of the centre obtained from a single partition $R \vdash n$ is

$$
z_{R}=\sum_{\sigma \in S_{n}} \chi^{R}(\sigma) \sigma \otimes \sigma
$$

One can quickly verify that $z_{R_{1}, R_{2} ; R_{3}}=z_{R_{1}, R_{2}} \cdot z_{R_{3}} \in \mathcal{K}(n)$ :

$$
\begin{aligned}
(\gamma \otimes \gamma) \cdot z_{R_{1}, R_{2} ; R_{3}} \cdot\left(\gamma^{-1} \otimes \gamma^{-1}\right) & =\sum_{\sigma_{i} \in S_{n}} \chi^{R_{1}}\left(\sigma_{1}\right) \chi^{R_{2}}\left(\sigma_{2}\right) \chi^{R_{3}}\left(\sigma_{3}\right) \gamma \sigma_{1} \sigma_{3} \gamma^{-1} \otimes \gamma \sigma_{2} \sigma_{3} \gamma^{-1} \\
& =\sum_{\sigma_{i} \in S_{n}} \chi^{R_{1}}\left(\sigma_{1}\right) \chi^{R_{2}}\left(\sigma_{2}\right) \chi^{R_{3}}\left(\sigma_{3}\right) \gamma \sigma_{1} \sigma_{3} \gamma^{-1} \otimes \gamma \sigma_{2} \sigma_{3} \gamma^{-1} \\
& =\sum_{\sigma_{i} \in S_{n}} \chi^{R_{1}}\left(\sigma_{1}\right) \chi^{R_{2}}\left(\sigma_{2}\right) \chi^{R_{3}}\left(\sigma_{3}\right) \sigma_{1} \sigma_{3} \otimes \sigma_{2} \sigma_{3}=z_{R_{1}, R_{2} ; R_{3}}
\end{aligned}
$$

where we change variables $\gamma \sigma_{i=1,2} \rightarrow \tilde{\sigma}_{i=1,2} \gamma, \gamma \sigma_{3} \gamma^{-1} \rightarrow \tilde{\sigma}_{3}$, and rename $\tilde{\sigma}_{i=1,2,3}$ as $\sigma_{i=1,2,3}$.

We arrive at the following statement:

Proposition 5. The set $\left\{z_{R_{1}, R_{2} ; R_{3}}\right\}$, with $R_{i} \vdash n$, is an overcomplete basis of the centre $\mathcal{Z}(\mathcal{K}(n))$.

Proof. We project $P^{R_{1}, R_{2}, R_{3}}$ onto $z_{R_{1}^{\prime}, R_{2}^{\prime} ; R_{3}^{\prime}}$ and check that the coefficients are not vanishing (see details in (B.17) in appendix B.1):

$$
\boldsymbol{\delta}_{2}\left(P^{R_{1}, R_{2}, R_{3}} ; z_{R_{1}^{\prime}, R_{2}^{\prime} ; R_{3}^{\prime}}\right)=n ! \delta_{R_{1} R_{1}^{\prime}} \delta_{R_{2} R_{2}^{\prime}} \delta_{R_{3} R_{3}^{\prime}} \mathrm{C}\left(R_{1}, R_{2}, R_{3}\right)
$$

Hence $P^{R_{1}, R_{2}, R_{3}}$ admits a decomposition in terms of $z_{R_{1}^{\prime}, R_{2}^{\prime} ; R_{3}^{\prime}}$. The overcompleteness follows from the number of elements of $\left\{z_{R_{1}^{\prime}, R_{2}^{\prime} ; R_{3}^{\prime}}\right\}$ is $p(n)^{3}$ which is larger than the number of non vanishing Kroneckers the dimension of $\mathcal{Z}(\mathcal{K}(n))$.

A general study of central elements in algebras constructed as subgroup-centralizers in a group algebra is given in [62]. 


\subsection{Double coset algebra}

The algebra $\mathcal{K}(n)$ introduced in the previous sections as a sub-algebra of $\mathbb{C}\left(S_{n}\right) \otimes \mathbb{C}\left(S_{n}\right)$ has another description as a sub-algebra of $\mathbb{C}\left(S_{n}\right) \otimes \mathbb{C}\left(S_{n}\right) \otimes \mathbb{C}\left(S_{n}\right)$. As we will see shortly, in this latter description, we have an algebra of double cosets. The former description as a centralizer algebra is a gauge-fixed version. Hence the double coset description is an un-gauge-fixed version. For this reason, we will refer to the double coset algebra in this section as $\mathcal{K}_{\text {un }}(n)$ and establish its isomorphism with $\mathcal{K}(n)$. In the rest of the paper, we will use $\mathcal{K}(n)$ for either description of the algebra, and it will be clear from the context whether we are working with the gauge-fixed (centralizer algebra) or un-gauge-fixed (double coset) description. While the centralizer algebra is a more economical description, being embedded in a smaller algebra, $\mathcal{K}_{\text {un }}(n)$ arises more immediately from inspection of the permutation equivalences relevant to tensor models, as reviewed in section 2 .

$\mathcal{K}_{\text {un }}(\boldsymbol{n})$ as a double coset algebra in $\mathbb{C}\left(\boldsymbol{S}_{\boldsymbol{n}}\right)^{\otimes \mathbf{3}}$. Consider elements $\sigma_{1} \otimes \sigma_{2} \otimes \sigma_{3} \in$ $\mathbb{C}\left(S_{n}\right)^{\otimes 3}$ and the left and right actions of $\operatorname{Diag}\left(\mathbb{C}\left(S_{n}\right)\right)$ on these triples as:

$$
\sigma_{1} \otimes \sigma_{2} \otimes \sigma_{3} \rightarrow \sum_{\gamma_{1}, \gamma_{2} \in S_{n}} \gamma_{1} \sigma_{1} \gamma_{2} \otimes \gamma_{1} \sigma_{2} \gamma_{2} \otimes \gamma_{1} \sigma_{3} \gamma_{2}
$$

$\mathcal{K}_{\text {un }}(n)$ is the vector space and sub-algebra of $\mathbb{C}\left(S_{n}\right) \otimes \mathbb{C}\left(S_{n}\right) \otimes \mathbb{C}\left(S_{n}\right)$ which is invariant under left and right actions by the diagonal $\operatorname{Diag}\left(\mathbb{C}\left(S_{n}\right)\right)$ :

$$
\mathcal{K}_{\text {un }}(n)=\operatorname{Span}_{\mathbb{C}}\left\{\sum_{\gamma_{1}, \gamma_{2} \in S_{n}} \gamma_{1} \sigma_{1} \gamma_{2} \otimes \gamma_{1} \sigma_{2} \gamma_{2} \otimes \gamma_{1} \sigma_{3} \gamma_{2}, \sigma_{1}, \sigma_{2}, \sigma_{3} \in S_{n}\right\}
$$

The equivalence classes defining $\mathcal{K}_{\text {un }}(n)$ are the double cosets

$$
\operatorname{Diag}\left(S_{n}\right) \backslash\left(S_{n} \times S_{n} \times S_{n}\right) / \operatorname{Diag}\left(S_{n}\right)
$$

It is simple to check that $\mathcal{K}_{\text {un }}(n)$ is stable under multiplication. The identity of $\mathcal{K}_{\text {un }}(n)$ is $i d \otimes i d \otimes i d$. The rest of required properties to make $\mathcal{K}_{\text {un }}(n)$ a sub-algebra of $\mathbb{C}\left(S_{n}\right)^{\otimes 3}$ can be easily verified.

Proposition 6. $\mathcal{K}_{\mathrm{un}}(n)$ is an associative unital sub-algebra of $\mathbb{C}\left(S_{n}\right)^{\otimes 3}$.

In fact, one shows that the two algebras $\mathcal{K}(n)$ and $\mathcal{K}_{\text {un }}(n)$ have the same dimension. The isomorphism between the basis elements stems from a change or variable: $\gamma_{1} \rightarrow \gamma_{2}^{-1} \sigma_{1}^{-1}$, and then renaming $\sigma_{1}^{-1} \sigma_{i}$ as $\sigma_{i}, i=2,3$, and $\gamma_{2} \rightarrow \gamma$. Under this change of variable we obtain

$$
\sum_{\gamma_{1}, \gamma_{2} \in S_{n}} \gamma_{1} \sigma_{1} \gamma_{2} \otimes \gamma_{1} \sigma_{2} \gamma_{2} \otimes \gamma_{1} \sigma_{3} \gamma_{2}=\sum_{\gamma \in S_{n}} i d \otimes \gamma \sigma_{2} \gamma^{-1} \otimes \gamma \sigma_{3} \gamma^{-1}
$$

and the r.h.s. is clearly associated with the basis element $\sum_{\gamma \in S_{n}} \gamma \sigma_{2} \gamma^{-1} \otimes \gamma \sigma_{3} \gamma^{-1}$ of $\mathcal{K}(n)$. It is direct to get $\operatorname{dim} \mathcal{K}_{\text {un }}(n)=\operatorname{dim} \mathcal{K}(n)=Z_{3}(n)$. Finally, we will keep the name of "graphs" as elements of $\mathcal{K}_{\text {un }}(n)$. 
Fourier basis $Q_{\text {un }}$. In this formulation in terms of triples of permutations, the basis of invariants of $\mathcal{K}_{\text {un }}(n)$ is given by

$$
Q_{\mathrm{un} ; \tau_{1}, \tau_{2}}^{R, S, T}=\kappa_{R, S, T} \sum_{\sigma_{l} \in S_{n}} \sum_{i_{l}, j_{l}} C_{i_{1}, i_{2} ; i_{3}}^{R, S ; T, \tau_{1}} C_{j_{1}, j_{2} ; j_{3}}^{R, S ; T, \tau_{2}} D_{i_{1}, j_{1}}^{R}\left(\sigma_{1}\right) D_{i_{2}, j_{2}}^{S}\left(\sigma_{2}\right) D_{i_{3}, j_{3}}^{T}\left(\sigma_{3}\right) \sigma_{1} \otimes \sigma_{2} \otimes \sigma_{3}
$$

with $\kappa_{R, S, T}=\frac{d(R) d(S) d(T)}{(n !)^{3}}$. The basis $\left\{Q_{\mathrm{un} ; \tau_{1}, \tau_{2}}^{R, S, T}\right\}$ is called $Q_{\mathrm{un}}$-basis. Its elements are invariant under left and right diagonal actions (see (B.5) for an intermediate step, appendix B.1):

$$
\left(\gamma_{1}^{\otimes 3}\right) \cdot Q_{\mathrm{un} ; \tau_{1}, \tau_{2}}^{R, S, T} \cdot\left(\gamma_{2}^{\otimes 3}\right)=Q_{\mathrm{un} ; \tau_{1}, \tau_{2}}^{R, T}
$$

and multiply like matrices (for a few details, see (B.6) in appendix B.1):

$$
\begin{aligned}
& Q_{\mathrm{un} ; \tau_{1}, \tau_{2}}^{R, S, T} Q_{\mathrm{un} ; \tau_{2}, \tau_{3}}^{R^{\prime}, S^{\prime}}=\kappa_{R, S, T} \delta_{R, R^{\prime}} \delta_{S, S^{\prime}} \delta_{T, T^{\prime}} \\
& \quad \times \sum_{\sigma_{l} \in S_{n}} \sum_{i_{l}, j_{l}} \sum_{\sigma_{l}^{\prime} \in S_{n}} \sum_{a_{l}} C_{i_{1}, i_{2} ; i_{3}}^{R, S ; T, \tau_{1}} C_{a_{1}, a_{2} ; a_{3}}^{R, S ; T, \tau_{3}} D_{i_{1}, a_{1}}^{R}\left(\sigma_{1}\right) D_{i_{2}, a_{2}}^{S}\left(\sigma_{2}\right) D_{i_{3}, a_{3}}^{T}\left(\sigma_{3}\right) \sigma_{1} \otimes \sigma_{2} \otimes \sigma_{3} \\
& \quad \times \sum_{j_{l}} C_{j_{1}, j_{2} ; j_{3}}^{R, S ; T, \tau_{2}} C_{j_{1}, j_{2} ; j_{3}}^{R, S ; T, \tau_{2}}=\delta_{R, R^{\prime}} \delta_{S, S^{\prime}} \delta_{T, T^{\prime}} Q_{\mathrm{un} ; \tau_{1}, \tau_{3}}^{R, S, T}
\end{aligned}
$$

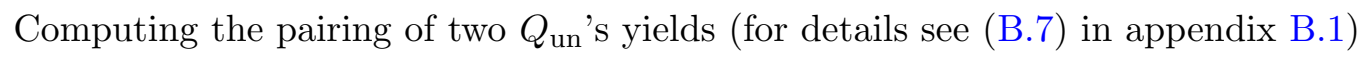

$$
\boldsymbol{\delta}_{3}\left(Q_{\mathrm{un} ; \tau_{1}, \tau_{2}}^{R, S} ; Q_{\mathrm{un} ; \tau_{1}^{\prime}, \tau_{2}^{\prime}}^{R^{\prime}, T^{\prime}, T^{\prime}}\right)=\kappa_{R, S, T} d(T)^{2} \delta_{R R^{\prime}} \delta_{S S^{\prime}} \delta_{T T^{\prime}} \delta_{\tau_{1} \tau_{1}^{\prime}} \delta_{\tau_{2} \tau_{2}^{\prime}}
$$

we infer that the basis $Q_{\text {un }}$ is orthogonal. Same comments about making $Q_{\text {un }}$ orthonormal by appropriately tuning the $\kappa_{R, S, T}$ normalization factor can be made at this stage.

The centre $\mathcal{Z}\left(\mathcal{K}_{\text {un }}(\boldsymbol{n})\right)$. We now investigate the centre $\mathcal{Z}\left(\mathcal{K}_{\text {un }}(n)\right)$ of $\mathcal{K}_{\text {un }}(n)$. Using the same strategy as in section 3.3, we construct now the basis of the centre by taking the trace of the matrices $Q_{\mathrm{un}}$ 's

$$
P_{\mathrm{un}}^{R, S, T}=\sum_{\tau} Q_{\mathrm{un} ; \tau, \tau}^{R, S, T}
$$

We show that $P_{\mathrm{un}}^{R, S, T}$ is commuting with any $Q_{\mathrm{un} ; \tau_{1}, \tau_{2}}^{R^{\prime}, S^{\prime}, T^{\prime}}$

$$
\begin{aligned}
P_{\mathrm{un}}^{R, S, T} \cdot Q_{\mathrm{un} ; \tau_{1}, \tau_{2}}^{R^{\prime}, S^{\prime}, T^{\prime}} & =\sum_{\tau} Q_{\mathrm{un} ; \tau, \tau}^{R, S, T} Q_{\mathrm{un} ; \tau_{1}, \tau_{2}}^{R^{\prime}, S^{\prime}, T^{\prime}} \\
& =\delta_{R, R^{\prime}} \delta_{S, S^{\prime}} \delta_{T, T^{\prime}} \sum_{\tau} \delta_{\tau, \tau_{1}} Q_{\mathrm{un} ; \tau, \tau_{2}}^{R, S, T}=\delta_{R, R^{\prime}} \delta_{S, S^{\prime}} \delta_{T, T^{\prime}} Q_{\mathrm{un} ; \tau_{1}, \tau_{2}}^{R, S, T} \\
Q_{\mathrm{un} ; \tau_{1}, \tau_{2}}^{R^{\prime}, S^{\prime}, T^{\prime}} \cdot P_{\mathrm{un}}^{R, S, T} & =\delta_{R, R^{\prime}} \delta_{S, S^{\prime}} \delta_{T, T^{\prime}} \sum_{\tau} \delta_{\tau, \tau_{2}} Q_{\mathrm{un} ; \tau_{1}, \tau}^{R, S, T}=P_{\mathrm{un}}^{R, S, T} \cdot Q_{\mathrm{un} ; \tau_{1}, \tau_{2}}^{R^{\prime}, S^{\prime}, T^{\prime}}
\end{aligned}
$$

Hence $P_{\mathrm{un}}^{R, S, T}$ is in the centre of $\mathcal{K}_{\mathrm{un}}(n)$. Adapting the arguments of the proof of Proposition 4 in the present context, the next result can be deduced without difficulties. 
Proposition 7. $\left\{P_{\mathrm{un}}^{R, S, T}\right\}$ is a basis of $\mathcal{Z}\left(\mathcal{K}_{\mathrm{un}}(n)\right)$.

The pairing of two $P_{\text {un }}$ 's gives

$$
\begin{aligned}
\delta_{3}\left(P_{\mathrm{un}}^{R, S, T} ; P_{\mathrm{un}}^{R^{\prime}, S^{\prime}, T^{\prime}}\right) & =\sum_{\tau, \tau^{\prime}} \delta_{3}\left(Q_{\mathrm{un} ; \tau, \tau}^{R, S, T} ; Q_{\mathrm{un} ; \tau^{\prime}, \tau^{\prime}}^{R^{\prime}, T^{\prime}, T^{\prime}}\right) \\
& =\kappa_{R, S, T} d(T)^{2} \delta_{R R^{\prime}} \delta_{S S^{\prime}} \delta_{T T^{\prime}} \sum_{\tau, \tau^{\prime}} \delta_{\tau \tau^{\prime}} \\
& =\kappa_{R, S, T} d(T)^{2} \mathrm{C}(R, S, T) \delta_{R R^{\prime}} \delta_{S S^{\prime}} \delta_{T T^{\prime}}
\end{aligned}
$$

Overcomplete bases of $\mathcal{Z}\left(\mathcal{K}_{\mathbf{u n}}(\boldsymbol{n})\right)$. Given three Young diagrams $R_{i}, i=1,2,3$, and the central element $z_{R_{i}}=\sum_{\sigma} \chi^{R_{i}}(\sigma) \sigma$ of $\mathbb{C}\left(S_{n}\right)$, we are interested by the element

$$
z_{\mathrm{un}}^{R_{1}, R_{2}, R_{3}}=z_{R_{1}} \otimes z_{R_{2}} \otimes z_{R_{2}}=\sum_{\sigma_{i} \in S_{n}} \chi^{R_{1}}\left(\sigma_{1}\right) \chi^{R_{2}}\left(\sigma_{2}\right) \chi^{R_{3}}\left(\sigma_{3}\right) \sigma_{1} \otimes \sigma_{2} \otimes \sigma_{3}
$$

that proves to belong to the centre of $\mathbb{C}\left(S_{n}\right)^{\otimes 3}$. It is sufficient to prove this claim for any basis element as

$$
\begin{aligned}
\gamma_{1} \otimes \gamma_{2} \otimes \gamma_{3} \cdot z_{\mathrm{un}}^{R_{1}, R_{2}, R_{3}} & =\sum_{\sigma_{i} \in S_{n}} \chi^{R_{1}}\left(\sigma_{1}\right) \chi^{R_{2}}\left(\sigma_{2}\right) \chi^{R_{3}}\left(\sigma_{3}\right) \gamma_{1} \sigma_{1} \otimes \gamma_{2} \sigma_{2} \otimes \gamma_{3} \sigma_{3} \\
& =\sum_{\sigma_{i} \in S_{n}} \chi^{R_{1}}\left(\gamma_{1}^{-1} \sigma_{1}\right) \chi^{R_{2}}\left(\gamma_{2}^{-1} \sigma_{2}\right) \chi^{R_{3}}\left(\gamma_{3}^{-1} \sigma_{3}\right) \sigma_{1} \otimes \sigma_{2} \otimes \sigma_{3} \\
& =\sum_{\sigma_{i} \in S_{n}} \chi^{R_{1}}\left(\sigma_{1} \gamma_{1}^{-1}\right) \chi^{R_{2}}\left(\sigma_{2} \gamma_{2}^{-1}\right) \chi^{R_{3}}\left(\sigma_{3} \gamma_{3}^{-1}\right) \sigma_{1} \otimes \sigma_{2} \otimes \sigma_{3} \\
& =\sum_{\sigma_{i} \in S_{n}} \chi^{R_{1}}\left(\sigma_{1}\right) \chi^{R_{2}}\left(\sigma_{2}\right) \chi^{R_{3}}\left(\sigma_{3}\right) \sigma_{1} \gamma_{1} \otimes \sigma_{2} \gamma_{2} \otimes \sigma_{3} \gamma_{3} \\
& =z_{\mathrm{un}}^{R_{1}, R_{2}, R_{3}} \cdot \gamma_{1} \otimes \gamma_{2} \otimes \gamma_{3}
\end{aligned}
$$

we used a change of variable $\sigma_{i} \rightarrow \gamma_{i}^{-1}$.

The following statement holds.

Proposition 8. $\left\{z_{\mathrm{un}}^{R_{1}, R_{2}, R_{3}}\right\}$ forms an overcomplete basis of the centre $\mathcal{Z}\left(\mathcal{K}_{\mathrm{un}}(n)\right)$.

Proof. We want to find an expansion

$$
P_{\mathrm{un}}^{R_{1}, R_{2}, R_{3}}=\sum_{R_{1}^{\prime}, R_{2}^{\prime}, R_{3}^{\prime}} \boldsymbol{\delta}_{3}\left(z_{\mathrm{un}}^{R_{1}^{\prime}, R_{2}^{\prime}, R_{3}^{\prime}} ; P_{\mathrm{un}}^{R_{1}, R_{2}, R_{3}}\right) z_{\mathrm{un}}^{R_{1}^{\prime}, R_{2}^{\prime}, R_{3}^{\prime}}
$$

with the coefficient $\boldsymbol{\delta}_{3}\left(z_{\mathrm{un}}^{R_{1}^{\prime}, R_{2}^{\prime}, R_{3}^{\prime}} ; P_{\mathrm{un}}^{R_{1}, R_{2}, R_{3}}\right)$. That quantity has been computed in (B.19) of appendix B.2 and one finds it as

$$
\boldsymbol{\delta}_{3}\left(z_{\mathrm{un}}^{R_{1}^{\prime}, R_{2}^{\prime}, R_{3}^{\prime}} ; P_{\mathrm{un}}^{R_{1}, R_{2}, R_{3}}\right)=d\left(R_{3}\right) \mathrm{C}\left(R_{1}, R_{2}, R_{3}\right) \delta_{R_{1} R_{1}^{\prime}} \delta_{R_{2} R_{2}^{\prime}} \delta_{R_{3} R_{3}^{\prime}}
$$

Now, for a given triple $\left(R_{1}, R_{2}, R_{3}\right)$ for which $P_{\text {un }}^{R_{1}, R_{2}, R_{3}}$ is not vanishing, then the coefficient $\delta_{3}\left(z_{\mathrm{un}}^{R_{1}^{\prime}, R_{2}^{\prime}, R_{3}^{\prime}} ; P_{\mathrm{un}}^{R_{1}, R_{2}, R_{3}}\right)$ is not vanishing. Therefore $P_{\mathrm{un}}^{R_{1}, R_{2}, R_{3}}$ has an expansion in terms of the $z_{\mathrm{un}}^{R_{1}, R_{2}, R_{3}}$ 's. The cardinality of $\left\{z_{\mathrm{un}}^{R_{1}, R_{2}, R_{3}}\right\}$ is $p(n)^{3}$, cube of the number of partitions of $n$, is larger than the number of nonvanishing Kroneckers $\mathrm{C}\left(R_{1}, R_{2}, R_{3}\right)$. The basis $\left\{z_{\mathrm{un}}^{R_{1}, R_{2}, R_{3}}\right\}$ is therefore overcomplete. 


\section{$4 \mathcal{K}(n)$ as a graph algebra}

As already mentioned, to each element of $\mathcal{K}_{\mathrm{un}}(n)$ of the form $\sum_{\gamma_{1}, \gamma_{2} \in S_{n}} \gamma_{1} \sigma_{1} \gamma_{2} \otimes \gamma_{1} \sigma_{2} \gamma_{2} \otimes$ $\gamma_{1} \sigma_{3} \gamma_{2}$, we associate a tensor observable, determined by the triple of permutations $\left(\sigma_{1}, \sigma_{2}, \sigma_{3}\right)$ subjected to the equivalence $\left(\sigma_{1}, \sigma_{2}, \sigma_{3}\right) \sim \gamma_{1} \cdot\left(\sigma_{1}, \sigma_{2}, \sigma_{3}\right) \cdot \gamma_{2}$. We now investigate the algebra inherited on colored bipartite graphs induced from the multiplication law of $\mathcal{K}_{\text {un }}(n)$. As observed in [41] and discussed earlier in this paper, the gauge-fixed formulation involves permutation pairs subject to simultaneous conjugation equivalence. These naturally correspond to ordinary bi-partite graphs (edges are not colored). A different algebra structure on the space of bi-partite graphs has been considered in [63].

First, given the normalized graph elements of $\mathcal{K}_{\mathrm{un}}(n)$, labelled by $\sigma_{i} \in S_{n}$,

$$
A_{\sigma_{1}, \sigma_{2}, \sigma_{3}}=\frac{1}{(n !)^{2}} \sum_{\gamma_{1}, \gamma_{2} \in S_{n}} \gamma_{1} \sigma_{1} \gamma_{2} \otimes \gamma_{1} \sigma_{2} \gamma_{2} \otimes \gamma_{1} \sigma_{3} \gamma_{2}
$$

we write a product of two of these elements in $\mathcal{K}_{\text {un }}(n)$ as

$$
A_{\sigma_{1}, \sigma_{2}, \sigma_{3}} A_{\sigma_{4}, \sigma_{5}, \sigma_{6}}=\frac{1}{(n !)^{4}} \sum_{\gamma_{1}, \gamma_{2}, \tau_{1}, \tau_{2} \in S_{n}} \gamma_{1} \sigma_{1} \gamma_{2} \tau_{1} \sigma_{4} \tau_{2} \otimes \gamma_{1} \sigma_{2} \gamma_{2} \tau_{1} \sigma_{5} \tau_{2} \otimes \gamma_{1} \sigma_{3} \gamma_{2} \tau_{1} \sigma_{6} \tau_{2}
$$

A change of variables $\gamma_{2} \tau_{1} \rightarrow \tau_{1}$, and renaming of $\tau_{2}$ as $\gamma_{2}$ and $\tau_{1}$ as $\tau$, allow us to get

$$
\begin{aligned}
A_{\sigma_{1}, \sigma_{2}, \sigma_{3}} A_{\sigma_{4}, \sigma_{5}, \sigma_{6}} & =\frac{1}{n !} \sum_{\tau \in S_{n}}\left[\frac{1}{(n !)^{2}} \sum_{\gamma_{1}, \gamma_{2} \in S_{n}} \gamma_{1}\left(\sigma_{1} \tau \sigma_{4}\right) \gamma_{2} \otimes \gamma_{1}\left(\sigma_{2} \tau \sigma_{5}\right) \gamma_{2} \otimes \gamma_{1}\left(\sigma_{3} \tau \sigma_{6}\right) \gamma_{2}\right] \\
& =\frac{1}{n !} \sum_{\tau \in S_{n}} A_{\sigma_{1} \tau \sigma_{4}, \sigma_{2} \tau \sigma_{5}, \sigma_{3} \tau \sigma_{6}}
\end{aligned}
$$

Thus, the product of two graphs can be written as a sum of graphs. There is a particular element such that

$$
\begin{aligned}
A_{\sigma_{1}, \sigma_{2}, \sigma_{3}} A_{i d, i d, i d} & =\frac{1}{n !} \sum_{\tau \in S_{n}}\left[\frac{1}{(n !)^{2}} \sum_{\gamma_{1}, \gamma_{2} \in S_{n}} \gamma_{1}\left(\sigma_{1} \tau\right) \gamma_{2} \otimes \gamma_{1}\left(\sigma_{2} \tau\right) \gamma_{2} \otimes \gamma_{1}\left(\sigma_{3} \tau\right) \gamma_{2}\right] \\
& =\frac{1}{(n !)^{2}} \sum_{\gamma_{1}, \gamma_{2} \in S_{n}} \gamma_{1} \sigma_{1} \gamma_{2} \otimes \gamma_{1} \sigma_{2} \gamma_{2} \otimes \gamma_{1} \sigma_{3} \gamma_{2}=A_{\sigma_{1}, \sigma_{2}, \sigma_{3}}
\end{aligned}
$$

and similarly $A_{i d, i d, i d} A_{\sigma_{1}, \sigma_{2}, \sigma_{3}}=A_{\sigma_{1}, \sigma_{2}, \sigma_{3}}$. This shows that $A_{i d, i d, i d}=E$ is a unit element of the graph algebra.

In the gauge-fixed formulation, the graph multiplication takes the form:

$$
\begin{aligned}
B_{\sigma_{1}, \sigma_{2}} B_{\sigma_{3}, \sigma_{4}} & =\sum_{\gamma_{1}, \gamma_{2}} \gamma_{1} \sigma_{1} \gamma_{1}^{-1} \gamma_{2} \sigma_{3} \gamma_{2}^{-1} \otimes \gamma_{1} \sigma_{2} \gamma_{1}^{-1} \gamma_{2} \sigma_{4} \gamma_{2}^{-1} \\
& =\sum_{\tau, \gamma_{2}} \gamma_{2} \tau^{-1} \sigma_{1} \tau \sigma_{3} \gamma_{2}^{-1} \otimes \gamma_{2} \tau^{-1} \sigma_{2} \tau \sigma_{4} \gamma_{2}^{-1} \\
& =\sum_{\tau} B_{\tau^{-1}} \sigma_{1} \tau \sigma_{3}, \tau^{-1} \sigma_{2} \tau \sigma_{4}
\end{aligned}
$$


where we used a change of variables $\gamma_{1} \rightarrow \gamma_{2} \tau^{-1}$ and omit normalization factor, for simplicity. This relation can be also obtained from (4.3) after some proper gauge fixing.

Coming back to the formula (4.3), we can illustrate this in diagram
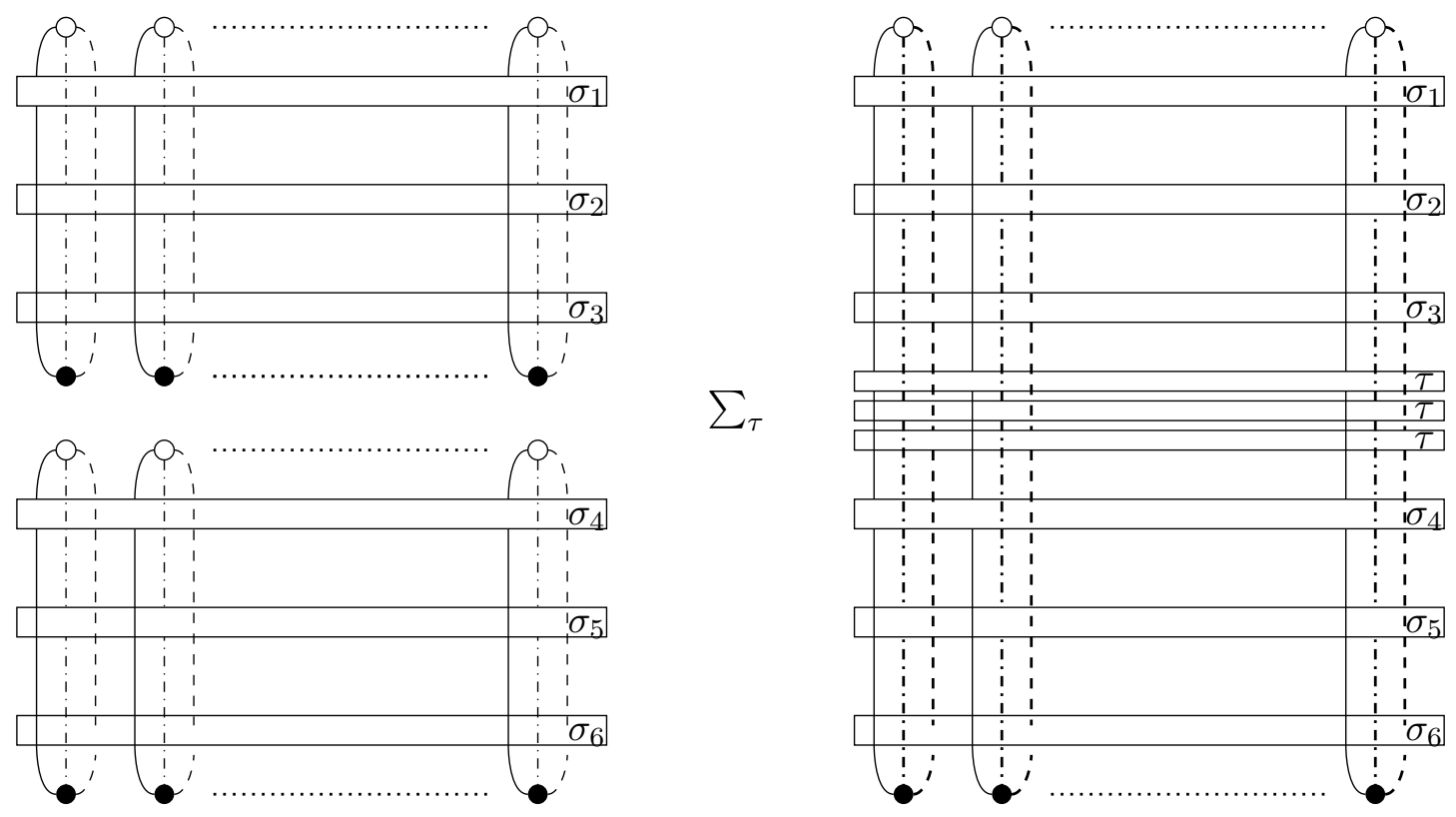

Algebra $\mathcal{K}_{\text {un }}(\boldsymbol{n}=\mathbf{1})$. Let us illustrate the formula (4.3) at $n=1$. There is no choice here, we obtain $E^{2}=E$. This is the unique invariant made by contraction of two tensors, that is $\sum_{n_{1}, n_{2}, n_{3}} T_{n_{1}, n_{2}, n_{3}} \bar{T}_{n_{1}, n_{2}, n_{3}}=E$. We consider $E$ as an idempotent or unit element of an 1 dimensional algebra $\{E\}$.

Algebra $\mathcal{K}_{\text {un }}(\boldsymbol{n}=\mathbf{2})$. We now examine $n=2$. There are 4 possible diagrams, see (4.7).

$$
\begin{gathered}
E \sim\left(\sigma_{1}=i d, \sigma_{2}=i d, \sigma_{3}=i d\right)= \\
A_{i d, i d,(12)}=A \sim\left(\sigma_{1}=i d, \sigma_{2}=i d, \sigma_{3}=(12)\right)= \\
A_{i d,(12), i d}=B \sim\left(\sigma_{1}=i d, \sigma_{2}=(12), \sigma_{3}=i d\right)= \\
A_{(12), i d, i d}=D \sim\left(\sigma_{1}=(12), \sigma_{2}=i d, \sigma_{3}=i d\right)=
\end{gathered}
$$
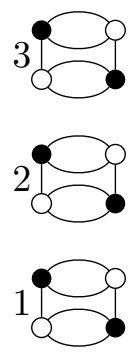

where the labels 3,2,1 denote a particular colored edge which can be used as a label of the invariant. Note that due to the equivalence under left and right diagonal action, any other choice reduces to one of the above. For example $\sigma_{1}=i d, s_{2}=(12), s_{3}=(12) \sim(12) \cdot\left(\sigma_{1}=\right.$ $\left.i d, s_{2}=(12), s_{3}=(12)\right) \cdot(12)=\left(\sigma_{1}=(12), \sigma_{2}=i d, \sigma_{3}=i d\right)$. We then compute some 
products (note that they are normalized by $1 /(2 !)^{2}$ and we use $(4.3)$ ):

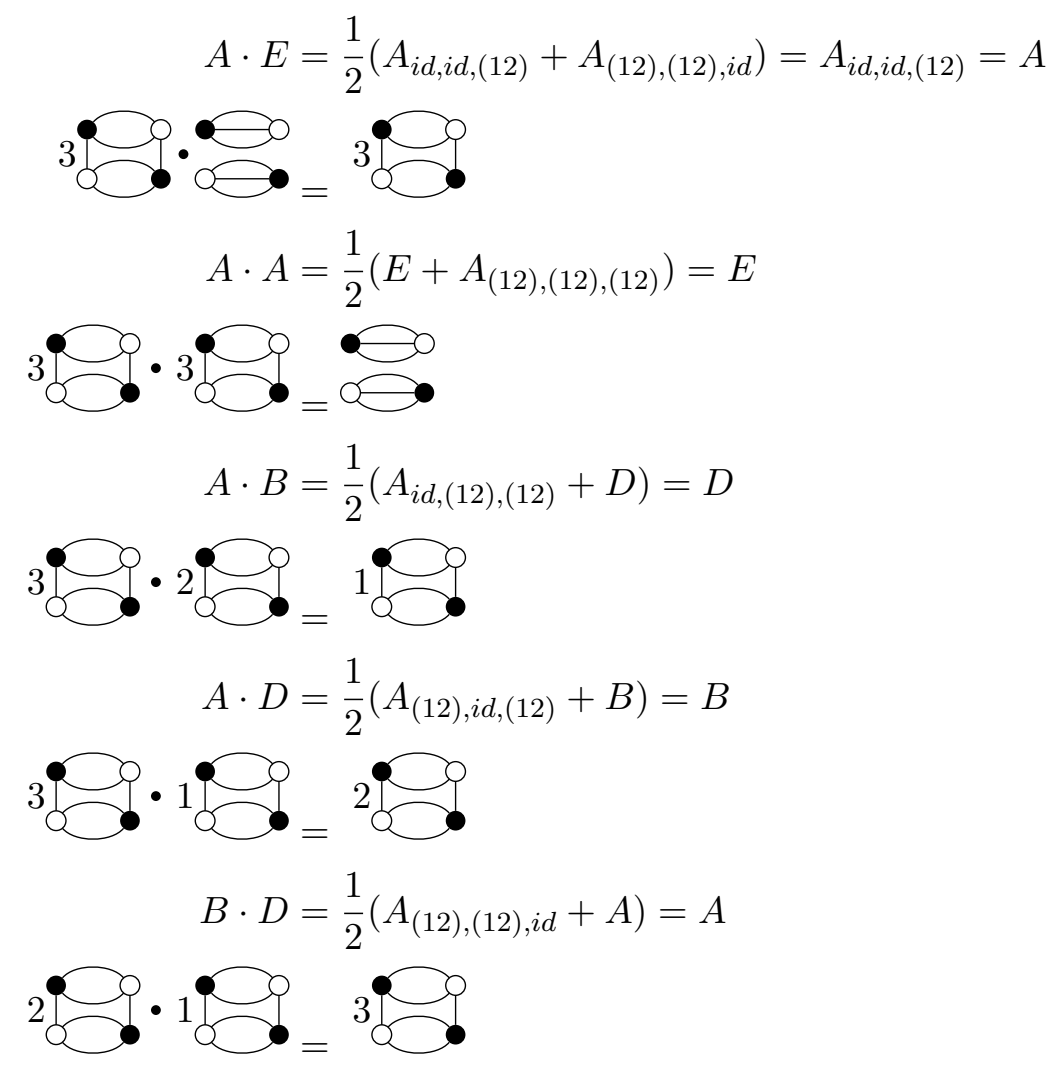

Other products behave like, in loose notations, $B E=B, D E=D, B^{2}=E, D^{2}=$ $E, A B=B A=D, A D=D A=B, B D=D B=A$. Thus $E$ is the unit element of the multiplication law. Furthermore, it is simple to check that the law its associative $(A B) D=D^{2}=E=A^{2}=A(B D),(A B) B=D B=A=(A E)=A\left(B^{2}\right)$, commutative and any element of the graph basis is its own inverse. As expected $\mathcal{K}(2)=\mathbb{C}\left(S_{2}\right) \otimes \mathbb{C}\left(S_{2}\right)$ : the diagonal conjugation action which defines $\mathcal{K}(2)$ leaves the permutation pairs invariant.

Algebra $\mathcal{K}_{\text {un }}(\boldsymbol{n}=\mathbf{3})$. The number of invariants is $Z_{3}(3)=11$ and this makes the multiplication table more complicated. We have listed the products in appendix C. In fact, 21 products involving the unit $E=\stackrel{\ominus}{\ominus}$ are known.

From the multiplication table, we see that $\mathcal{K}_{\text {un }}(3)$ is commutative, that some basis elements can be factorized. We illustrate the product for a non trivial situation obtained by taking the product of the following elements $A_{(12),(123), i d}$ and $A_{(123),(123),(12)}$ depicted as:

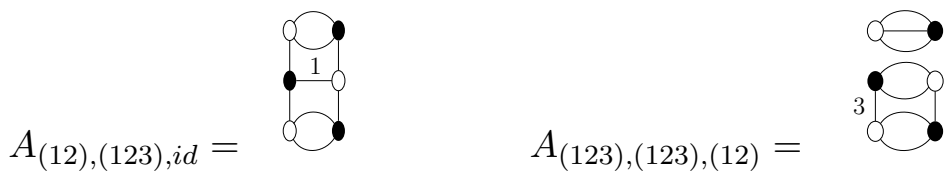


Then we get:

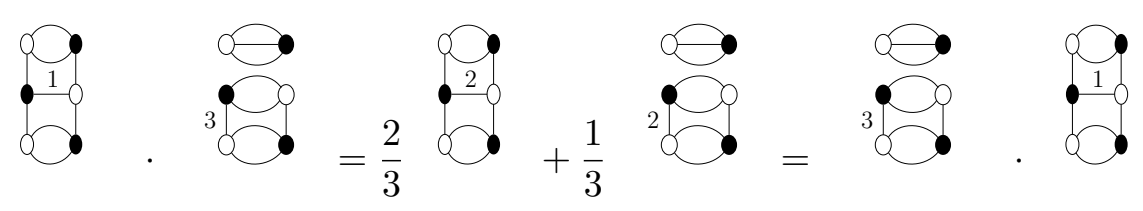

\section{PCAs and correlators}

In this section, we undertake the analysis of correlators of Gaussian tensor models, building on the permutation description for tensor model observables introduced in [41] and reviewed earlier. We start with one-point functions of tensor model observables corresponding to central elements in $\mathcal{K}(n)$. These observables are labelled by triples of Young diagrams and are sums of permutation basis operators weighted by characters. As explained in section 3 such sums of permutations weighted by characters lead to an overcomplete basis for the centre in $\mathcal{K}(n)$. Correlators parametrized by Young diagrams using characters have also been highlighted in $[58,59]$. Analogous correlators at higher $d$ are expressed in terms of sums of products of Kronecker coefficients. In section 5.2, we use known results on Kronecker coefficients to give explicit formulae for several families of correlators. In section 5.3 we consider normal ordered 2-point correlators, which we have briefly discussed in [41]. We show that the tensor model observables corresponding to the WA basis for $\mathcal{K}(n)$ discussed in section 3 provide an orthogonal basis for these 2-point functions. This orthogonality property has also been considered in $[38,59]$.

\subsection{Correlators for central observables}

We start our analysis with correlators of general observables at $d=3$, parametrised by permutations, corresponding to general elements of $\mathcal{K}(n)$. We then specialize to central observables labelled by triples of Young diagrams: as we saw in section 3 triples of projectors labelled by Young diagrams lead to an overcomplete basis for the centre $\mathcal{Z}(\mathcal{K}(n))$. We extend the discussion to any $d$.

Rank $\boldsymbol{d}=\mathbf{3}$ correlator. In rank $d=3$ tensor models, consider a general observable $\mathcal{O}_{\sigma_{1}, \sigma_{2}, \sigma_{3}}$ defined by three permutations $\sigma_{i}, i=1,2,3$. The expectation value $\left\langle\mathcal{O}_{\sigma_{1}, \sigma_{2}, \sigma_{3}}\right\rangle$ evaluates in the Gaussian measure, using appendix D. We write:

$$
\begin{aligned}
& \left\langle\mathcal{O}_{\sigma_{1}, \sigma_{2}, \sigma_{3}}\right\rangle=\sum_{\gamma} N^{\mathbf{c}\left(\gamma \sigma_{1}\right)+\mathbf{c}\left(\gamma \sigma_{2}\right)+\mathbf{c}\left(\gamma \sigma_{3}\right)} \\
& =\sum_{\gamma} \sum_{\alpha_{1}, \alpha_{2}, \alpha_{3}} N^{\mathbf{c}\left(\alpha_{1}\right)+\mathbf{c}\left(\alpha_{2}\right)+\mathbf{c}\left(\alpha_{3}\right)} \delta\left(\gamma \sigma_{1} \alpha_{1}\right) \delta\left(\gamma \sigma_{2} \alpha_{2}\right) \delta\left(\gamma \sigma_{3} \alpha_{3}\right) \\
& =\sum_{\gamma} \sum_{\alpha_{l}} \sum_{R_{l}} \frac{d\left(R_{1}\right) d\left(R_{2}\right) d\left(R_{3}\right)}{(n !)^{3}} N^{\mathbf{c}\left(\alpha_{1}\right)+\mathbf{c}\left(\alpha_{2}\right)+\mathbf{c}\left(\alpha_{3}\right)} \chi^{R_{1}}\left(\gamma \sigma_{1} \alpha_{1}\right) \chi^{R_{2}}\left(\gamma \sigma_{2} \alpha_{2}\right) \chi^{R_{3}}\left(\gamma \sigma_{3} \alpha_{3}\right)
\end{aligned}
$$

where we expand the $\delta$ 's over $S_{n}$ using characters as in (A.16) of appendix A.1. Now we use three facts: (1) $\sum_{\alpha} N^{\mathbf{c}_{\alpha}} \alpha$ is a central element in $\mathbb{C}\left(S_{n}\right)$, since $\mathbf{c}\left(g \alpha \gamma^{-1}\right)=\mathbf{c}(\alpha),(2)$ if $B$ is a central element, characters factorize as $\chi(A B)=\frac{1}{d(R)} \chi^{R}(A) \chi^{R}(B)$, see (A.20), 
appendix A.1, and (3) that characters extend by linearity over $\mathbb{C}\left(S_{n}\right), \chi^{R}\left(\sum_{\gamma} c_{\gamma} \gamma\right)=$ $\sum_{\gamma} c_{\gamma} \chi^{R}(\gamma)$, to write $(5.1)$ as

$$
\begin{aligned}
& \left\langle\mathcal{O}_{\sigma_{1}, \sigma_{2}, \sigma_{3}}\right\rangle=\sum_{\gamma} \sum_{R_{l}} \frac{1}{(n !)^{3}} d\left(R_{1}\right) d\left(R_{2}\right) d\left(R_{3}\right) \\
& \quad \times \chi^{R_{1}}\left(\gamma \sigma_{1}\left(\sum_{\alpha_{1}} N^{\mathbf{c}\left(\alpha_{1}\right)} \alpha_{1}\right)\right) \chi^{R_{2}}\left(\gamma \sigma_{2}\left(\sum_{\alpha_{2}} N^{\mathbf{c}\left(\alpha_{2}\right)} \alpha_{2}\right)\right) \chi^{R_{3}}\left(\gamma \sigma_{3}\left(\sum_{\alpha_{3}} N^{\mathbf{c}\left(\alpha_{3}\right)} \alpha_{3}\right)\right) \\
& =\sum_{\gamma} \sum_{R_{l}} \frac{1}{(n !)^{3}} \chi^{R_{1}}\left(\gamma \sigma_{1}\right) \chi^{R_{2}}\left(\gamma \sigma_{2}\right) \chi^{R_{3}}\left(\gamma \sigma_{3}\right) \sum_{\alpha_{l}} N^{\mathbf{c}\left(\alpha_{1}\right)+\mathbf{c}\left(\alpha_{2}\right)+\mathbf{c}\left(\alpha_{3}\right)} \chi^{R_{1}}\left(\alpha_{1}\right) \chi^{R_{2}}\left(\alpha_{2}\right) \chi^{R_{3}}\left(\alpha_{3}\right) \\
& =\sum_{\gamma} \sum_{R_{l}}\left[\prod_{l=1}^{3} \operatorname{Dim}_{N}\left(R_{l}\right)\right] \chi^{R_{1}}\left(\gamma \sigma_{1}\right) \chi^{R_{2}}\left(\gamma \sigma_{2}\right) \chi^{R_{3}}\left(\gamma \sigma_{3}\right)
\end{aligned}
$$

where, in the last stage, we use (A.23) of appendix A.1. $\operatorname{Dim}_{N}(R)$ is the dimension of the representation of the unitary group $\mathrm{U}(N)$ determined by the Young tableau $R$. Consider sums of $\mathcal{O}_{\sigma_{1}, \sigma_{2}, \sigma_{3}}$ weighted by characters with Young diagrams $S_{l} \vdash n, l=1,2,3$, and define the function

$$
\left\langle\mathcal{O}_{S_{1}, S_{2}, S_{3}}\right\rangle=\frac{1}{(n !)^{3}} \sum_{\sigma_{l} \in S_{n}} \chi^{S_{1}}\left(\sigma_{1}\right) \chi^{S_{2}}\left(\sigma_{2}\right) \chi^{S_{3}}\left(\sigma_{3}\right)\left\langle\mathcal{O}_{\sigma_{1}, \sigma_{2}, \sigma_{3}}\right\rangle
$$

These observables correspond to central elements in $\mathcal{K}(n)$ by the map (2.7). We can use character orthogonality (see (A.18) and (A.1))

$$
\sum_{\sigma} \chi^{S}(\sigma) \chi^{R}(\sigma \gamma)=\frac{n !}{d(R)} \delta^{R, S} \chi^{R}(\gamma)
$$

to write

$$
\begin{aligned}
\left\langle\mathcal{O}_{S_{1}, S_{2}, S_{3}}\right\rangle & =\left[\prod_{l=1}^{3} \frac{\operatorname{Dim}_{N}\left(S_{l}\right)}{d\left(S_{l}\right)}\right] \sum_{\gamma} \chi^{S_{1}}(\gamma) \chi^{S_{2}}(\gamma) \chi^{S_{3}}(\gamma)=n !\left[\prod_{l=1}^{3} \frac{\operatorname{Dim}_{N}\left(S_{l}\right)}{d\left(S_{l}\right)}\right] \mathrm{C}\left(S_{1}, S_{2}, S_{3}\right) \\
& =\frac{1}{(n !)^{2}}\left[\prod_{l=1}^{3} f_{N}\left(S_{l}\right)\right] \mathrm{C}\left(S_{1}, S_{2}, S_{3}\right)
\end{aligned}
$$

Thus the correlators $\left\langle\mathcal{O}_{S_{1}, S_{2}, S_{3}}\right\rangle$ are proportional to the Kronecker coefficients. The factors $f_{N}\left(S_{i}\right)$ are products of box weights of the Young diagrams (A.2). In a large $N$ limit where we are considering tensor invariants of degree $n$, hence Young diagrams with $n$ boxes, where $n$ is kept fixed and $N$ is taken to infinity, the $f$-factors behave like $N^{n}$ at leading order. The relative magnitudes of the correlators in this limit is determined purely by the Kronecker coefficients. At finite $N$, since we are dealing with a theory where the tensor indices are taking $N$ possible values, the Young diagrams are cut-off to have no more than $N$ rows.

Rank $\boldsymbol{d}$ correlator. The above formula (5.2) can be generalized at any rank $d$. Rank $d$ Gaussian correlators of a generic observables $\mathcal{O}_{\sigma_{1}, \sigma_{2}, \ldots, \sigma_{d}}$ labelled by $d$ permutations $\sigma_{l}$, 
$l=1,2, \ldots, d$. We will sketch the above analysis for $\mathcal{O}_{\sigma_{1}, \sigma_{2}, \ldots, \sigma_{d}}$ :

$$
\begin{aligned}
\left\langle\mathcal{O}_{\sigma_{1}, \sigma_{2}, \ldots, \sigma_{d}}\right\rangle & =\sum_{\gamma} N^{\mathbf{c}\left(\gamma \sigma_{1}\right)+\mathbf{c}\left(\gamma \sigma_{2}\right)+\cdots+\mathbf{c}\left(\gamma \sigma_{d}\right)} \\
& =\sum_{\gamma} \sum_{R_{l}} \frac{1}{(n !)^{d}}\left[\prod_{l=1}^{d} d\left(R_{l}\right) \chi^{R_{l}}\left(\gamma \sigma_{l} \sum_{\alpha_{l}} N^{\mathbf{c}\left(\alpha_{l}\right)} \alpha_{l}\right)\right]
\end{aligned}
$$

Then, using the same technique, we arrive at

$$
\left\langle\mathcal{O}_{\sigma_{1}, \sigma_{2}, \ldots, \sigma_{d}}\right\rangle=\sum_{\gamma} \sum_{R_{l}}\left[\prod_{l=1}^{d} \operatorname{Dim}_{N}\left(R_{l}\right) \chi^{R_{l}}\left(\gamma \sigma_{l}\right)\right]
$$

We calculate the Fourier transform of $\mathcal{O}_{\sigma_{1}, \sigma_{2}, \ldots, \sigma_{d}}$ weighted by characters. Let $S_{l}$, $l=1, \ldots, d$, partitions of $n$,

$$
\begin{aligned}
\left\langle\mathcal{O}_{S_{1}, S_{2}, \ldots, S_{d}}\right\rangle & =\sum_{\sigma_{1}, \sigma_{2}, \ldots, \sigma_{d}} \frac{1}{(n !)^{d}} \chi^{S_{1}}\left(\sigma_{1}\right) \chi^{S_{2}}\left(\sigma_{2}\right) \ldots \chi^{S_{d}}\left(\sigma_{d}\right)\left\langle\mathcal{O}_{\sigma_{1}, \sigma_{2}, \ldots, \sigma_{d}}\right\rangle \\
& =\left[\prod_{l=1}^{d} \frac{\operatorname{Dim}_{N}\left(S_{l}\right)}{d\left(S_{l}\right)}\right] \sum_{\gamma}\left[\prod_{l=1}^{d} \chi^{S_{l}}(\gamma)\right]
\end{aligned}
$$

Introducing $\mathrm{C}_{d}\left(S_{1}, S_{2}, \ldots, S_{d}\right)=\frac{1}{n !} \sum_{\gamma}\left[\prod_{l=1}^{d} \chi^{S_{l}}(\gamma)\right]$, the number of invariants in $S_{1} \otimes$ $S_{2} \otimes \cdots \otimes S_{d}$, we can write

$$
\left\langle\mathcal{O}_{S_{1}, S_{2}, \ldots, S_{d}}\right\rangle=n !\left[\prod_{l=1}^{d} \frac{\operatorname{Dim}_{N}\left(S_{l}\right)}{d\left(S_{l}\right)}\right] \mathrm{C}_{d}\left(S_{1}, S_{2}, \ldots, S_{d}\right)
$$

As an illustration, restricting to rank $d=4$, and using the relation (3.9), that is $\mathrm{C}_{4}\left(S_{1}, S_{2}, S_{3}, S_{4}\right)=\sum_{S} \mathrm{C}\left(S_{1}, S_{2}, S\right) \mathrm{C}\left(S, S_{3}, S_{4}\right)$ counting the number of invariants in $S_{1} \otimes$ $S_{2} \otimes S_{3} \otimes S_{4}$, we have

$$
\left\langle\mathcal{O}_{S_{1}, S_{2}, S_{3}, S_{4}}\right\rangle=n !\left[\prod_{l=1}^{4} \frac{\operatorname{Dim}_{N}\left(S_{l}\right)}{d\left(S_{l}\right)}\right] \sum_{S} \mathrm{C}\left(S_{1}, S_{2}, S\right) \mathrm{C}\left(S, S_{3}, S_{4}\right)
$$

Note that $\mathrm{C}_{d}\left(S_{1}, \ldots, S_{d}\right)$ can be decomposed as a sum of Kronecker coefficients convoluted in one of their indices. A possible sequence of such a convolution could be $\sum_{\bar{S}_{l}} \mathrm{C}\left(S_{1}, S_{2}, \bar{S}_{1}\right)$ $\mathrm{C}\left(\bar{S}_{1}, S_{3}, \bar{S}_{2}\right) \mathrm{C}\left(\bar{S}_{2}, S_{4}, \bar{S}_{3}\right) \mathrm{C}\left(\bar{S}_{3}, S_{5}, \bar{S}_{4}\right) \ldots$ Any permutation over $S_{i}$ 's giving a different sequence should give the same answer $\mathrm{C}_{d}\left(S_{1}, \ldots, S_{d}\right)$. Then, we observe that there is graphical way to encode the expansion of $\left\langle\mathcal{O}_{S_{1}, S_{2}, \ldots, S_{d}}\right\rangle$ as a convoluted sum of Kronecker coefficient $\mathrm{C}\left(S_{a}, S_{b}, S_{c}\right)$. Reminiscent of Feynman rules, we associate $\mathrm{C}\left(S_{a}, S_{b}, S_{c}\right)$ with a trivalent graph vertices and half edges labelled by $S_{a}, S_{b}$ and $S_{c}$, each symbol $S$ summed over between two Kroneckers $\mathrm{C}\left(S_{a}, S_{b}, S\right)$ and $\mathrm{C}\left(S, S_{b^{\prime}}, S_{c^{\prime}}\right)$ is associated with an edge between the vertices $\mathrm{C}\left(S_{a}, S_{b}, S\right)$ and $\mathrm{C}\left(S, S_{b^{\prime}}, S_{c^{\prime}}\right)$. It is not hard to realize that the corresponding graph is always a tree graph with vertex set with vertices of degree 3 and $d$ half-edges. Therefore, each correlator $\left\langle\mathcal{O}_{S_{1}, S_{2}, \ldots, S_{d}}\right\rangle$ is associated with a decomposition in several tree 

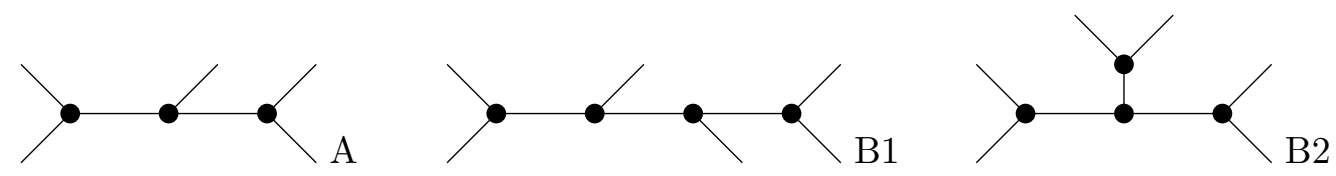

Figure 2. Order 5 unlabelled tree configurations.

graphs the half edges of which are labelled by $S_{1}, \ldots, S_{d}$. Any of these tree graphs to which we finally give a weight $n !\left[\prod_{l=1}^{d} \frac{\operatorname{Dim}_{N}\left(S_{l}\right)}{d\left(S_{l}\right)}\right]$ is a valid representative of $\left\langle\mathcal{O}_{S_{1}, S_{2}, \ldots, S_{d}}\right\rangle$. For example, the correlator (5.10) is associated with any of the following trees:
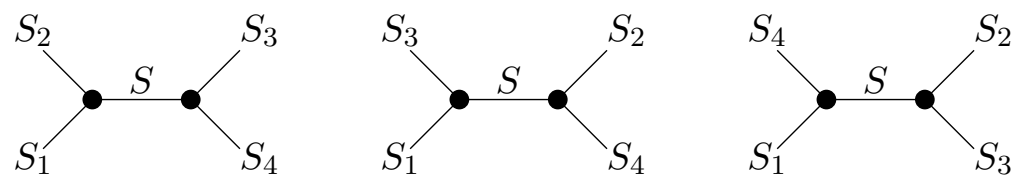

At order 5 , there is a unique unlabelled tree configuration (see figure 2 A) yielding 15 different tree labellings of half edges (or leaves) and, at order 6 , there are 2 unlabelled tree configurations (see, figure 2 B1 and B2) giving 120 different tree labellings of half edges (B1 yields 90, and B2,30). The counting of that type of trees is the counting of 3-regular (or binary) trees with $d$ leaves and $d-2$ vertices (and so $2 d-3$ edges). This will involve a mixture of a counting of the so-called binary beanstalk (A and B1) but also more general terms. For $d=3,4,5,6$, we have the sequence

$$
1,3,15,120
$$

respectively, which should be completed at any $d$.

\subsection{Correlators and Kronecker coefficients: explicit examples}

To illustrate the above formula (5.5), we evaluate correlators of rank 3 tensor models as a function of $N>1$, and $n \geq 0$, for some particular Young diagrams.

For any $S_{2}$ and $S_{3}$, and for $S_{1}=[n]=\underbrace{\begin{array}{|l|l|l|}\hline & & \\ \hline\end{array}}_{n \text {-boxes }}$, then $\mathrm{C}\left(S_{1}, S_{2}, S_{3}\right)=1$ and so $\left\langle\mathcal{O}_{S_{1}, S_{2}, S_{3}}\right\rangle=\left[\prod_{l=1}^{3} f_{N}\left(S_{l}\right)\right] /(n !)^{2}$, from (5.5). That computes to

$$
\left\langle\mathcal{O}_{S_{1}, S_{2}, S_{3}}\right\rangle=(N-2)(N-3) \ldots(N-1-n) \frac{f_{N}\left(S_{2}\right) f_{N}\left(S_{3}\right)}{(n !)^{2}}
$$

Note that, in the following, we consider that $N$ is large enough compared to $n$. Specifying $S_{2}$ and $S_{3}$ to give a more precise formula for the correlator. For all $n$, consider the Young diagrams defined by

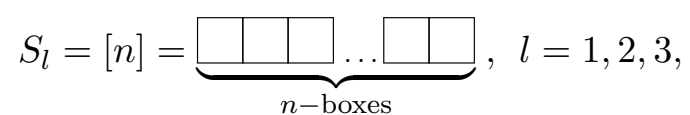

such that from (5.13), one gets

$$
\left\langle\mathcal{O}_{S_{1}, S_{2}, S_{3}}\right\rangle=\frac{[(N-2)(N-3) \ldots(N-1-n)]^{3}}{(n !)^{2}}=\frac{[(N-2) !]^{3}}{(n !)^{2}[(N-2-n) !]^{3}}
$$

Varying the order of the symmetric group, that is varying $n=1,2,3, \ldots, 10$ gives table 1 . 


\begin{tabular}{|c|c|c|c|c|c|}
\hline & $n=1$ & $\mathrm{n}=2$ & $n=3$ & $\mathrm{n}=4$ & $\mathrm{n}=5$ \\
\hline$N=2$ & 0 & 0 & - & - & - \\
\hline$N=3$ & 1 & 0 & 0 & - & - \\
\hline$N=4$ & 8 & 2 & 0 & 0 & - \\
\hline$N=5$ & 27 & 54 & 6 & 0 & 0 \\
\hline$N=6$ & 64 & 432 & 384 & 24 & 0 \\
\hline$N=7$ & 125 & 2000 & 6000 & 3000 & 120 \\
\hline$N=8$ & 216 & 6750 & 48000 & 81000 & 25920 \\
\hline$N=9$ & 343 & 18522 & 257250 & 1029000 & 1111320 \\
\hline$N=10$ & 512 & 43904 & 1053696 & 8232000 & 21073920 \\
\hline
\end{tabular}

Table 1. Evaluation of $\left\langle\mathcal{O}_{[n],[n],[n]}\right\rangle$.

Let us introduce the notation $[n-k, 1, \ldots, 1]=\left[n-k, 1^{k}\right]$, where $k$ is the number of 1 appearing in the dots. For all $n, \mathrm{C}\left([n],\left[n-k, 1^{k}\right],\left[n-k, 1^{k}\right]\right)=1$. Hence, for $k \in$ $\{0,1, \cdots, n-1\}$,

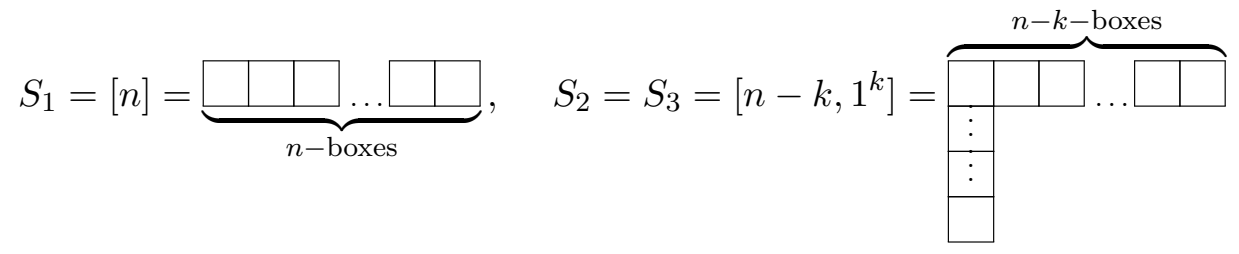

where the dots in the 1st vertical column refers to $k$-times a block of size 1 . Hence for this class of Young diagrams, the correlator calculation can be easily made.

$$
\begin{aligned}
\left\langle\mathcal{O}_{[n],\left[n-k, 1^{k}\right],\left[n-k, 1^{k}\right]}\right\rangle=(N-2)(N-3) \ldots(N-1-n) & \\
& \times \frac{\left[(N-2)(N-3)^{2} \ldots(N-1-k)^{2}(N-k-2)^{2}(N-k-3) \ldots(N-1-(n-k))\right]^{2}}{(n !)^{2}} \\
= & \frac{[(N-2)(N-3) \ldots(N-1-(n-k))]^{3}}{(n !)^{2}} \\
& \times(N-3)^{2}(N-4)^{2} \ldots(N-2-k)^{2}(N-n+k-2)(N-n+k-3) \ldots(N-1-n) \\
= & \frac{[(N-2) !]^{3}\left[\prod_{l=1}^{k}(N-2-l)^{2}\right]\left[\prod_{l=1}^{k}(N-n+k-1-l)\right]}{(n !)^{2}[(N-n+k-2) !]^{3}}
\end{aligned}
$$

It turns out that $\left\langle\mathcal{O}_{[n],\left[n-k, 1^{k}\right],\left[n-k, 1^{k}\right]}\right\rangle$ is not necessarily an integer for any values of $k$. One can check this by direct evaluation for instance using $N=7, n=3,\left\langle\mathcal{O}_{[3],[2,1],[2,1]}\right\rangle=\frac{32000}{3}$. In any case, we further restrict to the case $k=1$ and give $\left\langle\mathcal{O}_{[n],[n-1,1],[n-1,1]}\right\rangle$ for different values of $N$ and $n \geq 2$, in table 2 .

Next, we relax the assumption that $S_{1}$ is the symmetric representation. Avoiding trivial cases, consider $n \geq 2$ and $k \leq \frac{n}{2}$, then we consider the following

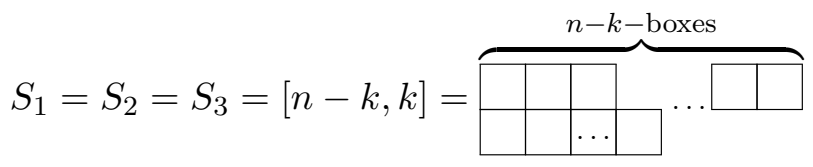

where of course the second row has $k$ boxes. 


\begin{tabular}{|c|c|c|c|c|c|}
\hline$N=2$ & $\mathrm{n}=2$ & $\mathrm{n}=3$ & $\mathrm{n}=4$ & $\mathrm{n}=5$ & $\mathrm{n}=6$ \\
$N=3$ & 0 & - & - & - & - \\
$N=4$ & 2 & 0 & - & - & - \\
$N=5$ & 54 & 24 & 0 & - & - \\
$N=6$ & 432 & 864 & 216 & 0 & - \\
$N=7$ & 2000 & $\frac{32000}{3}$ & 12000 & 1920 & 0 \\
$N=8$ & 6750 & 75000 & 225000 & 162000 & 18000 \\
\hline
\end{tabular}

Table 2. Evaluation of $\left\langle\mathcal{O}_{[n],[n-1,1],[n-1,1]}\right\rangle$.

\begin{tabular}{|c|c|c|c|c|c|}
\hline$N=2$ & $\mathrm{n}=2$ & $\mathrm{n}=3$ & $\mathrm{n}=4$ & $\mathrm{n}=5$ & $\mathrm{n}=6$ \\
$N=3$ & 0 & - & - & - & - \\
$N=4$ & 2 & 0 & - & - & - \\
$N=5$ & 54 & 48 & 0 & - & - \\
$N=6$ & 432 & 1296 & 648 & $\frac{648}{25}$ & - \\
$N=7$ & 2000 & $\frac{128000}{9}$ & 24000 & 7680 & $\frac{640}{3}$ \\
$N=8$ & 6750 & 93750 & 375000 & 405000 & 90000 \\
$N=9$ & 18522 & 444528 & 3472875 & 8890560 & 6667920 \\
\hline
\end{tabular}

Table 3. Evaluation of $\left\langle\mathcal{O}_{[n-1,1],[n-1,1],[n-1,1]}\right\rangle$.

According to a computation using SAGE mathematical software up to order $n=25$, for all $n \geq 3 k$, the Kronecker coefficient of the two-row Young diagrams is given by

$$
\mathrm{C}([n-k, k],[n-k, k],[n-k, k])=\left\lfloor\frac{k}{2}\right\rfloor+1
$$

where $\lfloor\cdot\rfloor$ denotes the floor function. Therefore, from (5.5), we have

$$
\left\langle\mathcal{O}_{[n-k, k],[n-k, k],[n-k, k]}\right\rangle=\frac{\left[\left(\prod_{l=1}^{n-k}(N-1-l)\right)\left(\prod_{l=1}^{k}(N-2-l)\right)\right]^{3}}{(n !)^{2}}\left(\left\lfloor\frac{k}{2}\right\rfloor+1\right)
$$

for $n \leq 25$ and $k \leq \frac{n}{3}$ : while we have checked for $n \leq 25$, we expect this will hold for higher $n$ as well. General stability properties of Kronecker coefficients are described in [64, 65]. This correlator (5.20) is not integral in general. We obtain table 3 listing some values of $\left\langle\mathcal{O}_{[n-1,1],[n-1,1],[n-1,1]}\right\rangle($ at $k=1)$. Note that for this table, the column $n=2$ coincides with the column $n=2$ of table 2 as expected from the correlator formulas (5.20) and (5.17) at $n=2$ and $k=1$.

Finally, let us consider three Young diagrams with rectangular shape. Consider $n$ to be divisible such that $n=j k$, with $j$ and $k$ integers:

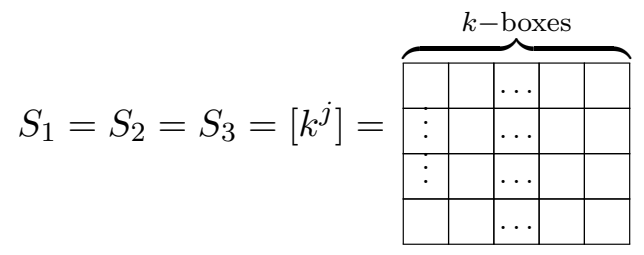




\begin{tabular}{|c|c|c|c|c|c|c|c|}
\hline & $\mathrm{n}=2$ & $\mathrm{n}=4$ & $\mathrm{n}=6$ & $\mathrm{n}=8$ & & $\mathrm{n}=3$ & $\mathrm{n}=6$ \\
\hline$N=2$ & - & - & 0 & - & $N=2$ & - & - \\
\hline$N=3$ & 0 & - & 0 & - & $N=3$ & - & - \\
\hline$N=4$ & 2 & 0 & 0 & - & $N=4$ & $\frac{2}{9}$ & - \\
\hline$N=5$ & 54 & 3 & 0 & - & $N=5$ & 6 & - \\
\hline$N=6$ & 432 & 648 & 0 & - & $N=6$ & 48 & - \\
\hline$N=7$ & 2000 & 24000 & 0 & - & $N=7$ & $\frac{2000}{9}$ & $\frac{80}{3}$ \\
\hline$N=8$ & 6750 & 375000 & 0 & $\frac{2430000}{49}$ & $N=8$ & 750 & $\frac{1250}{3}$ \\
\hline$N=9$ & 18522 & 3472875 & 0 & 17010000 & $N=9$ & 2058 & $\frac{15435}{4}$ \\
\hline
\end{tabular}

Table 4. Evaluation of $\left\langle\mathcal{O}_{\left[k^{2}\right],\left[k^{2}\right],\left[k^{2}\right]}\right\rangle$, for $n=2 k$ (left) and of $\left\langle\mathcal{O}_{\left[k^{3}\right],\left[k^{3}\right],\left[k^{3}\right]}\right\rangle$, with $n=3 k$ (right).

For simplicity, let us assume that $j \leq k<N-1$,

$$
\left\langle\mathcal{O}_{\left[k^{j}\right],\left[k^{j}\right],\left[k^{j}\right]}\right\rangle=\frac{1}{(n !)^{2}}\left[\prod_{l=1}^{k} \frac{(N-1-l) !}{(N-j-1-l) !}\right]^{3} \mathrm{C}\left(\left[k^{j}\right],\left[k^{j}\right],\left[k^{j}\right]\right)
$$

which can be again computed. We will restrict to the lowest orders in $k$ :

- If the number of rows is $j=2$, such that $S_{l}=\left[k^{2}\right]$, for $k$ even, we have $\mathrm{C}\left(\left[k^{2}\right],\left[k^{2}\right],\left[k^{2}\right]\right)=1$ and for $k$ odd, $\mathrm{C}\left(\left[k^{2}\right],\left[k^{2}\right],\left[k^{2}\right]\right)=0$. In this case, the correlator values coincide with columns $n=2$ and 4 of table 3 but, at order $n=8$, they start to differ. In this case the correlator is also not an integer. We obtain the l.h.s. of table 4 .

- If the number of rows is $j=3$, such that $S_{l}=\left[k^{3}\right]$, we have, for $k \leq 30$ and for $k$ even, $\mathrm{C}\left(\left[k^{3}\right],\left[k^{3}\right],\left[k^{3}\right]\right)=k / 2$ and for $k$ odd, $\mathrm{C}\left(\left[k^{3}\right],\left[k^{3}\right],\left[k^{3}\right]\right)=\lfloor k / 2\rfloor+1$. We obtain the r.h.s. of table 4 .

\subsection{Orthogonality of two-point functions and WA basis for $\mathcal{K}(n)$}

If we consider correlators of normal-ordered operators (see appendix D), then we can write them in terms of the product in $\mathcal{K}(n)$ and the delta function

$$
\left\langle\mathcal{O}_{\sigma_{1}, \sigma_{2}, \sigma_{3}} \mathcal{O}_{\tau_{1}, \tau_{2}, \tau_{3}}\right\rangle=\sum_{\gamma_{1}, \gamma_{2}} N^{3 n} \boldsymbol{\delta}_{3}\left[\left(\sigma_{1} \otimes \sigma_{2} \otimes \sigma_{3}\right) \gamma_{1}^{\otimes 3}\left(\tau_{1} \otimes \tau_{2} \otimes \tau_{3}\right) \gamma_{2}^{\otimes 3}\left(\Omega_{1} \otimes \Omega_{2} \otimes \Omega_{3}\right)\right]
$$

with $\Omega_{i}=\sum_{\alpha_{i} \in S_{n}} N^{\mathbf{c}\left(\alpha_{i}\right)-n} \alpha_{i}$. An important property of the $\Omega_{i}$ 's is that they are central elements of $\mathbb{C}\left(S_{n}\right)$. Indeed, using $\mathbf{c}\left(\gamma \alpha \gamma^{-1}\right)=\mathbf{c}(\alpha)$, one finds that $\gamma \Omega_{i} \gamma^{-1}=\Omega_{i}$. The tensor product of $\Omega$ factors defines a central element in $\mathcal{K}(n)$, hence the product in (5.23) involves the triples of permutations defining the two observables and the central elements defined by the $\Omega$-factors. The above equation (5.23) was derived as eq. (42) in [41] (or (102) of the arXiv version)). There is a mistake in a following equation ((105) of the arXiv) which should be

$$
\left\langle\mathcal{O}_{\sigma_{1}, \sigma_{2}, \sigma_{3}} \mathcal{O}_{\tau_{1}, \tau_{2}, \tau_{3}}\right\rangle=n ! \sum_{\mu \in S_{n}} \boldsymbol{\delta}_{2}\left[\left(\beta_{2}^{-1} \mu^{-1} \alpha_{2} \mu \Omega_{2}\right) \otimes\left(\beta_{3}^{-1} \mu^{-1} \alpha_{3} \mu \Omega_{3}\right) \Delta\left(\Omega_{1}\right)\right]
$$


where $\alpha_{2}=\sigma_{1}^{-1} \sigma_{2}, \alpha_{3}=\sigma_{1}^{-1} \sigma_{3}, \beta_{2}=\tau_{1}^{-1} \tau_{2}, \beta_{3}=\tau_{1}^{-1} \tau_{3}$, and

$$
\Delta(\Omega)=\sum_{\alpha} N^{\mathbf{c}(\alpha)} \alpha \otimes \alpha
$$

The Fourier basis (or representation basis ) of operators is defined by

$$
\begin{aligned}
\mathcal{O}_{\tau_{1}, \tau_{2}}^{R, S, T} & =\sum_{\sigma_{1}, \sigma_{2}, \sigma_{3}} \boldsymbol{\delta}_{3}\left(Q_{\mathrm{un} ; \tau_{1}, \tau_{2}}^{R, S, T} \sigma_{1}^{-1} \otimes \sigma_{2}^{-1} \otimes \sigma_{3}^{-1}\right) \mathcal{O}_{\sigma_{1}, \sigma_{2}, \sigma_{3}} \\
& =\kappa_{R, S, T} \sum_{\sigma_{l}} \sum_{i_{l}, j_{l}} C_{i_{1}, i_{2} ; i_{3}}^{R, S ; T, \tau_{1}} C_{j_{1}, j_{2} ; j_{3}}^{R, S ; T \tau_{2}} D_{i_{1} j_{1}}^{R}\left(\sigma_{1}\right) D_{i_{2} j_{2}}^{S}\left(\sigma_{2}\right) D_{i_{3} j_{3}}^{S}\left(\sigma_{3}\right) \mathcal{O}_{\sigma_{1}, \sigma_{2}, \sigma_{3}}
\end{aligned}
$$

The conjugate operator is

$$
\begin{aligned}
\overline{\mathcal{O}_{\tau_{1}, \tau_{2}}^{R, S, T}} & =\kappa_{R, S, T} \sum_{\sigma_{l}} \sum_{i_{l}, j_{l}} C_{i_{1}, i_{2} ; i_{3}}^{R, S ; T, \tau_{1}} C_{j_{1}, j_{2} ; j_{3}}^{R, S ; T, \tau_{2}} D_{i_{1} j_{1}}^{R}\left(\sigma_{1}\right) D_{i_{2} j_{2}}^{S}\left(\sigma_{2}\right) D_{i_{3} j_{3}}^{S}\left(\sigma_{3}\right) \mathcal{O}_{\sigma_{1}, \sigma_{2}, \sigma_{3}} \\
& =\kappa_{R, S, T} \sum_{\sigma_{l}} \sum_{i_{l}, j_{l}} C_{i_{1}, i_{2} ; i_{3}}^{R, S ; T, \tau_{1}} C_{j_{1}, j_{2} ; j_{3}}^{R, S ; T, \tau_{2}} D_{i_{1} j_{1}}^{R}\left(\sigma_{1}\right) D_{i_{2} j_{2}}^{S}\left(\sigma_{2}\right) D_{i_{3} j_{3}}^{S}\left(\sigma_{3}\right) \mathcal{O}_{\sigma_{1}^{-1}, \sigma_{2}^{-1}, \sigma_{3}^{-1}} \\
& =\kappa_{R, S, T} \sum_{\sigma_{l}} \sum_{i_{l}, j_{l}} C_{i_{1}, i_{2} ; i_{3}}^{R, S ; T, \tau_{1}} C_{j_{1}, j_{2} ; j_{3}}^{R, S ; T, \tau_{2}} D_{j_{1} i_{1}}^{R}\left(\sigma_{1}\right) D_{j_{2} i_{2}}^{S}\left(\sigma_{2}\right) D_{j_{3} i_{3}}^{S}\left(\sigma_{3}\right) \mathcal{O}_{\sigma_{1}, \sigma_{2}, \sigma_{3}} \\
& =\mathcal{O}_{\tau_{2}, \tau_{1}}^{R, S, T}
\end{aligned}
$$

where in the last stage of the equality, we simply rename $i_{l} \rightarrow j_{l}$ and vice-versa.

The two-point correlator evaluates as:

$$
\left\langle\mathcal{O}_{\tau_{1}, \tau_{1}^{\prime}}^{R_{1}, S_{1}, T_{1}} \overline{\mathcal{O}_{\tau_{2}, \tau_{2}^{\prime}}^{R_{2}, S_{2}, T_{2}}}\right\rangle=\sum_{\gamma_{1}, \gamma_{2}} N^{3 n} \boldsymbol{\delta}_{3}\left[Q_{\mathrm{un} ; \tau_{1}, \tau_{1}^{\prime}}^{R_{1}, S_{1}, T_{1}} \gamma_{1}^{\otimes 3} Q_{\mathrm{un} ; \tau_{2}^{\prime}, \tau_{2}}^{R_{2}, S_{2}, T_{2}} \gamma_{2}^{\otimes 3}\left(\Omega_{1} \otimes \Omega_{2} \otimes \Omega_{3}\right)\right]
$$

This shows that the inner product on tensor model observables, given by the Gaussian integral (with a normal ordering prescription which excludes Wick contractions within the observable), is proportional to the group theoretic inner product on $\mathcal{K}(n)$ with the insertion of the $\Omega_{1} \otimes \Omega_{2} \otimes \Omega_{3}$ factor. The invariance property of the $Q_{\mathrm{un}}$-operators (3.44) gives

$$
\left\langle\mathcal{O}_{\tau_{1}, \tau_{1}^{\prime}}^{R_{1}, S_{1}, T_{1}} \overline{\mathcal{O}_{\tau_{2}, \tau_{2}^{\prime}}^{R_{2}, S_{2}, T_{2}}}\right\rangle=(n !)^{2} N^{3 n} \boldsymbol{\delta}_{3}\left(Q_{\mathrm{un} ; \tau_{1}, \tau_{1}^{\prime}}^{R_{1}, S_{1}, T_{1}} Q_{\mathrm{un} ; \tau_{2}^{\prime}, \tau_{2}}^{R_{2}, S_{2}, T_{2}} \Omega_{1} \otimes \Omega_{2} \otimes \Omega_{3}\right)
$$

Use the matrix-multiplication property of the $Q_{\text {un-operators from (3.45) to write: }}$

$$
\left\langle\mathcal{O}_{\tau_{1}, \tau_{1}^{\prime}}^{R_{1}, S_{1}, T_{1}} \overline{\mathcal{O}_{\tau_{2}, \tau_{2}^{\prime}}^{R_{2}, S_{2}, T_{2}}}\right\rangle=(n !)^{2} N^{3 n} \delta_{R_{1}, R_{2}} \delta_{S_{1}, S_{2}} \delta_{T_{1}, T_{2}} \delta_{\tau_{1}^{\prime}, \tau_{2}^{\prime}} \boldsymbol{\delta}_{3}\left(Q_{\mathrm{un} ; \tau_{1}, \tau_{2}}^{R_{1}, S_{1}, T_{1}} \Omega_{1} \otimes \Omega_{2} \otimes \Omega_{3}\right)
$$

Since $\Omega_{i}$ are central elements in $\mathbb{C}\left(S_{n}\right)$, we have

$$
\begin{aligned}
Q_{\mathrm{un} ; \tau_{1}, \tau_{2}}^{R_{1}, R_{2}, R_{3}} \Omega_{1} \otimes \Omega_{2} \otimes \Omega_{3} & =\frac{\chi^{R_{1}}\left(\Omega_{1}\right)}{d\left(R_{1}\right)} \frac{\chi^{R_{2}}\left(\Omega_{2}\right)}{d\left(R_{2}\right)} \frac{\chi^{R_{3}}\left(\Omega_{3}\right)}{d\left(R_{3}\right)} Q_{\mathrm{un} ; \tau_{1}, \tau_{2}}^{R_{1}, R_{2}, R_{3}} \\
& =N^{-3 n} \operatorname{Dim}_{N}\left(R_{1}\right) \operatorname{Dim}_{N}\left(R_{2}\right) \operatorname{Dim}_{N}\left(R_{3}\right) Q_{\tau_{1}, \tau_{2}}^{R_{1}, R_{2}, R_{3}}
\end{aligned}
$$

where, recalling the form of $\chi^{R}(\Omega)=\sum_{\alpha} N^{\mathbf{c}(\alpha)-n} \chi^{R}(\alpha)$, we then use (A.25) in appendix A.1. 
We also have (as we prove shortly)

$$
\delta_{3}\left(Q_{\mathrm{un} ; \tau_{1}, \tau_{2}}^{R, S, T}\right)=\kappa_{R, S, T} \sum_{i_{l}, j_{l}} C_{i_{1}, i_{2} ; i_{3}}^{R, \tau_{1}} C_{j_{1}, j_{2} ; j_{3}}^{R, \tau_{2}} \delta_{i_{1}, j_{1}} \delta_{i_{2}, j_{2}} \delta_{i_{3}, j_{3}}=\kappa_{R, S, T} d(T) \delta_{\tau_{1}, \tau_{2}}
$$

The $Q$ 's behave like elementary matrices of a matrix algebra. The $\boldsymbol{\delta}_{3}$ behaves like the trace.

Using (5.30) and the above, the correlator becomes

$$
\begin{aligned}
& \left\langle\mathcal{O}_{\tau_{1}, \tau_{1}^{\prime}}^{R_{1}, T_{1}} \overline{\mathcal{O}_{\tau_{2}, \tau_{2}^{\prime}}^{R_{2}, S_{2}, T_{2}}}\right\rangle \\
& \quad=\delta_{R_{1}, R_{2}} \delta_{S_{1}, S_{2}} \delta_{T_{1}, T_{2}} \delta_{\tau_{1}, \tau_{2}} \delta_{\tau_{1}^{\prime}, \tau_{2}^{\prime}} \kappa_{R_{1}, S_{1}, T_{1}} d\left(T_{1}\right) \operatorname{Dim}_{N}\left(R_{1}\right) \operatorname{Dim}_{N}\left(S_{1}\right) \operatorname{Dim}_{N}\left(T_{1}\right)
\end{aligned}
$$

and this shows that $\left\{\mathcal{O}_{\tau, \tau^{\prime}}^{R, S}\right\}$ forms an orthogonal basis for Gaussian correlators (with normal ordered prescription) arising directly from the $Q_{\mathrm{un} ; \tau, \tau^{\prime}}^{R, S, T}$, which are the representation theoretic WA basis of $\mathcal{K}(n)$, via the map (2.7).

\section{$5.4 \mathcal{K}^{\infty}$ and correlators}

We have emphasized, in the bulk of the paper, the role of the algebra $\mathcal{K}(n)$ in organizing several aspects of correlators. Similar algebras arise in the context of matrix theory problems. For the half-BPS sector, we have the centre of $\mathbb{C}\left(S_{n}\right)$, denoted by $\mathcal{Z}\left(\mathbb{C}\left(S_{n}\right)\right)$. A basis at fixed $n$ is labelled by partitions, which in turn can be used to organize multitrace holomorphic functions of a single matrix of degree $n$ (i.e. a multi-trace containing $n$ copies of the matrix $Z$ ). The multiplication of two multi-traces of degrees $n_{1}$ and $n_{2}$ produces a multi-trace of degree $n_{1}+n_{2}$. Using the map to central elements of $\mathcal{Z}\left(\mathbb{C}\left(S_{n}\right)\right)$, this corresponds to an outer product which takes central elements $T_{1} \in \mathcal{Z}\left(\mathbb{C}\left(S_{n_{1}}\right)\right)$ and $T_{2} \in \mathcal{Z}\left(\mathbb{C}\left(S_{n_{1}}\right)\right)$ to get something in $\mathcal{Z}\left(\mathbb{C}\left(S_{n_{1}+n_{2}}\right)\right)$ followed by a projection to the centre. The direct sum

$$
\bigoplus_{n=0}^{\infty} \mathcal{Z}\left(\mathbb{C}\left(S_{n}\right)\right)
$$

provides a natural setting for correlators.

For the 2-matrix problem, relevant to the quarter BPS sector of $N=4 \mathrm{SYM}$, there is a PCA $\mathcal{A}(m, n)$ corresponding to multi-traces with $m$ copies of $X$ and $n$ copies of $Y$. In this case too, it is natural to consider a direct sum over all $m, n \geq 0$.

For the problem which is our present main focus, rank- 3 complex tensors, the analogous infinite dimensional associative algebra is

$$
\mathcal{K}^{\infty}=\bigoplus_{n=0}^{\infty} \mathcal{K}(n)
$$

There are in fact two associative products on this vector space. The product at fixed $n$ has been the main subject of this paper. The WA decomposition at fixed $n$ is related to the formula for $\mathcal{K}(n)$ as a sum of squares of $\mathrm{C}(R, S, T)$. There is also an outer product on 
$\mathcal{K}^{\infty}$ which we now decribe. Given two permutation pairs in $\mathcal{K}\left(n_{1}\right)$ and $\mathcal{K}\left(n_{2}\right)$ respectively,

$$
\begin{aligned}
& \left(\bar{\sigma}_{1}, \bar{\sigma}_{2}\right)=\sum_{\gamma_{1} \in S_{n_{1}}}\left(\gamma_{1} \sigma_{1} \gamma_{1}^{-1}, \gamma_{1} \sigma_{2} \gamma_{1}^{-1}\right) \\
& \left(\bar{\tau}_{1}, \bar{\tau}_{2}\right)=\sum_{\gamma_{2} \in S_{n_{2}}}\left(\gamma_{2} \tau_{1} \gamma_{2}^{-1}, \gamma_{2} \tau_{2} \gamma_{2}^{-1}\right)
\end{aligned}
$$

we have an outer product

$$
\begin{aligned}
\circ: \mathcal{K}\left(n_{1}\right) \otimes \mathcal{K}\left(n_{2}\right) & \rightarrow \mathcal{K}\left(n_{1}+n_{2}\right) \\
\left(\bar{\sigma}_{1}, \bar{\sigma}_{2}\right) \circ\left(\bar{\tau}_{1}, \bar{\tau}_{2}\right) & =\sum_{\gamma \in S_{n_{1}+n_{2}}}\left(\gamma\left(\bar{\sigma}_{1} \circ \bar{\tau}_{1}\right) \gamma^{-1}, \gamma\left(\bar{\sigma}_{2} \circ \bar{\tau}_{2}\right) \gamma^{-1}\right)
\end{aligned}
$$

This outer product to the multiplication of the gauge-invariant functions of $\Phi, \bar{\Phi}$ of degrees $n_{1}$ and $n_{2}$ to give a function of degree $n_{1}+n_{2}$. This outer product is related to the ring structure which has been described in detail, using the representation basis in [38].

As a generalization of normal ordered two-point functions,

$$
\left\langle\mathcal{N}\left(\mathcal{O}_{\sigma_{1}, \sigma_{2}}\right) \mathcal{N}\left(\mathcal{O}_{\tau_{1}, \tau_{2}}\right)\right\rangle
$$

which is expressed using the product in $\mathcal{K}(n)$, we may consider

$$
\left\langle\mathcal{N}\left(\mathcal{O}_{\sigma_{1}, \sigma_{2}} \mathcal{O}_{\tau_{1}, \tau_{2}}\right) \mathcal{N}\left(\mathcal{O}_{\sigma_{3}, \sigma_{4}}\right)\right\rangle
$$

Here $\sigma_{3}, \sigma_{4}$ are in $S_{n_{1}+n_{2}}$ and the correlator can be expressed in terms of

$$
\left(\left(\bar{\sigma}_{1}, \bar{\sigma}_{2}\right) \circ\left(\bar{\tau}_{1}, \bar{\tau}_{2}\right)\right) \cdot\left(\bar{\sigma}_{3}, \bar{\sigma}_{4}\right)
$$

which involves the commutative outer product followed by the non-commutative product within $\mathcal{K}\left(n_{1}+n_{2}\right)$. This interplay between the two products in the context of correlators of one and two-matrix models has been described in [52].

Infinite dimensional algebras constructed as direct sums of the above kind, with more than one associative product, in some cases with a co-product and Hopf algebra structure, have been studied in the subject of combinatorial Hopf algebras, with applications in diverse areas of combinatorics (see e.g. [66]). It would be interesting to explore the application of such combinatorial Hopf algebras in providing a mathematical framework for the computation of correlators in matrix/tensor models.

\section{Correlators, permutation-TFT2 and covers of 2-complexes}

In this section, we will show how correlators in tensor models can be expressed as observables in topological field theory of $S_{n}$ flat connections on appropriate 2-complexes. This $S_{n}$ description is closely related to covering spaces of the 2-complexes. The powers of $N$ are shown to be related to the Euler character of the cover. This generalizes to tensor models analogous results obtained for single-matrix models and quiver matrix models [57, 67]. Unlike the case of matrix models, where the 2-complexes are cell-decompositions of smooth 
2-manifolds (possibly with boundaries and possibly equipped with line defects), here the 2-complexes do not discretize smooth 2-dimensional spaces.

Consider (5.6) that we re-express:

$$
\begin{aligned}
\left\langle\mathcal{O}_{\sigma_{1}, \sigma_{2}, \cdots, \sigma_{d}}\right\rangle & =\sum_{\gamma, \alpha_{1}, \alpha_{2}, \cdots, \alpha_{d} \in S_{n}} \delta\left(\alpha_{1}^{-1} \gamma \sigma_{1}\right) \delta\left(\alpha_{2}^{-1} \gamma \sigma_{2}\right) \cdots \delta\left(\alpha_{d}^{-1} \gamma \sigma_{d}\right) N^{\mathbf{c}\left(\alpha_{1}\right)+\mathbf{c}\left(\alpha_{2}\right)+\cdots+\mathbf{c}\left(\alpha_{d}\right)} \\
& =\sum_{\alpha_{1}, \alpha_{2}, \cdots, \alpha_{d}} \delta\left(\alpha_{2} \alpha_{1} \sigma_{1}^{-1} \sigma_{2}\right) \delta\left(\alpha_{3} \alpha_{1} \sigma_{1}^{-1} \sigma_{3}\right) \cdots \delta\left(\alpha_{d} \alpha_{1} \sigma_{1}^{-1} \sigma_{d}\right)
\end{aligned}
$$

In the last line, we did the sum over $\gamma$ by using the first delta function. Defining $\tau_{1}=\sigma_{1}^{-1} \sigma_{2}$, $\tau_{2}=\sigma_{1}^{-1} \sigma_{3}, \cdots \tau_{d-1}=\sigma_{1}^{-1} \sigma_{2}$, we have

$$
\begin{aligned}
\left\langle\mathcal{O}_{\left.\sigma_{1}, \sigma_{2}, \cdots, \sigma_{d}\right)}\right. & =\left\langle\mathcal{O}_{1, \tau_{1}, \tau_{2}, \cdots, \tau_{d-1}}\right\rangle \\
& =\sum_{\alpha_{l}} \delta\left(\alpha_{2} \alpha_{1} \tau_{1}\right) \delta\left(\alpha_{3} \alpha_{1} \tau_{2}\right) \cdots \delta\left(\alpha_{d} \alpha_{1} \tau_{d-1}\right) N^{\mathbf{c}\left(\alpha_{1}\right)+\mathbf{c}\left(\alpha_{2}\right)+\cdots+\mathbf{c}\left(\alpha_{d}\right)}
\end{aligned}
$$

It is instructive to normalize the observables by including factors

$$
\prod_{i=1}^{d-1} N^{\left(\mathbf{c}\left(\tau_{i}\right)-3 n\right)}=N^{-2 n(d-1)} \prod_{i=1}^{d-1} N^{\left(\mathbf{c}\left(\tau_{i}\right)-n\right)}
$$

so that the normalized observable takes the form

$$
\widetilde{\mathcal{O}}_{1, \tau_{1}, \tau_{2}, \cdots, \tau_{d-1}}=\left(N^{-2 n(d-1)} \prod_{i=1}^{d-1} N^{\left(\mathbf{c}\left(\tau_{i}\right)-n\right)}\right) \mathcal{O}_{1, \tau_{1}, \tau_{2}, \cdots, \tau_{d-1}}
$$

We then write the above normalized correlator as

$$
\begin{aligned}
& \left\langle\widetilde{\mathcal{O}}_{1, \tau_{1}, \tau_{2}, \cdots, \tau_{d-1}}\right\rangle \\
& =\sum_{\alpha_{l}} N^{-n(d-1)+\sum_{i=1}^{d-1}\left(\mathbf{c}\left(\tau_{i}\right)-n\right)+\sum_{i=1}^{d}\left(\mathbf{c}\left(\alpha_{i}\right)-n\right)} \delta\left(\alpha_{2} \alpha_{1} \tau_{1}\right) \delta\left(\alpha_{3} \alpha_{1} \tau_{2}\right) \cdots \delta\left(\alpha_{d} \alpha_{1} \tau_{d-1}\right)
\end{aligned}
$$

These sums over delta functions can be interpreted as partition functions of twodimensional $S_{n}$ 2D-topological field theory (TFT2) on 2-complexes. These $S_{n}$ TFT2 are lattice gauge theories with a simple topological plaquette action consisting of delta functions for the product of permutations along the edges of the 2-cell. For a review of these TFT2 and their applications to correlators in quiver gauge theories and Feynman graph combinatorics see [57,68].

$S_{n}$ TFT2 are also closely related to branched covers, a fact which has applications in the string theory of $2 \mathrm{dYM}[69,70]$. It is interesting that it is possible to choose the normalizations of the operators and an overall normalization in terms of powers of $N$, such that the amplitude has a power of $N$ which is exactly equal to the Euler character of the covering 2-complex. The appropriate 2-complexes have a single vertex and loops corresponding to the permutations, and 2-cells (plaquettes) corresponding to the delta functions. Figure 3 shows the appropriate 2-cells for the cases $d=3,4$. The vertices, labelled $A$ are all identified to a single point. The $\alpha_{i}$ as well as the $\tau_{i}$ therefore correspond 

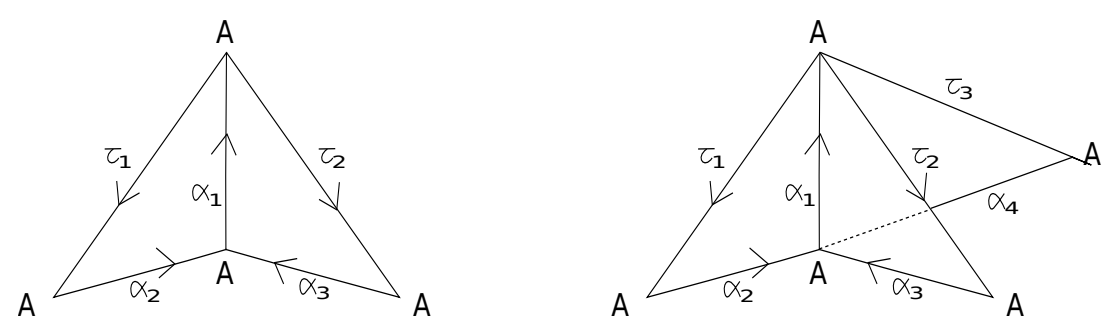

Figure 3. 2-cell for $S_{n}$ TFT2 interpretation of correlator at $d=3$ (left) and $d=4$ (right). The 2-cells in the 2-complexes are $\left(\alpha_{1} \tau_{l} \alpha_{l}\right), l=2,3,4$.

to closed loops. The triangles are the 2-cells. In the covering space interpretation, there is an $n$-fold cover of the 2-complex. The permutations $\alpha_{i}$ describe the monodromy of the sheets of the covering as the different cycles are traverses from $A$ back to $A$. The terms $(\mathbf{c}(\sigma)-n)$ is the contribution to the Euler character of a 2-surface whose boundary covers a circle on the target space with monodromy $\sigma$ (see for example the review on branched covers in $[70,71])$. Hence we have these factors for each of the $\alpha$ 's and $\tau$ 's. The 2-complex has one vertex, $2(d-1)+1=2 d-1$ edges, and $(d-1)$ faces. The Euler characteristic $V-E+F=-d+1$. According to the correlator formula above, when the permutations are all equal to the identity, the power is $-n(d-1)$, which is the correct Euler characteristic of the trivial $n$-fold cover.

The weight $N^{\chi}$ is expected from a string theory where $g_{s t}=N^{-1}$. The above formula for the correlators, as a sum over coverings of a 2-complex by a 2-complex, suggests an interpretation where the covering 2-complex is viewed as a "string worldsheet" and the target 2-complex as a target space for the string. Interestingly the 2-complexes for general $d$ have $d-12$-cells joined at the vertical edge. So these 2 -complexes are not cell decompositions of smooth two-dimensional surfaces. They can be 2 -skeletons for cell decompositions of 3-manifolds.

This raises a number of fascinating questions for the future. Is there some form of higher dimensional topological invariance in this string counting, associated with higher dimensional manifolds? Can we use this combinatoric string theory interpretation to develop a topological string action with these higher dimensional manifolds as target spaces? This logic of identifying the stringy geometry in the combinatorics of the large $N$ expansion proved useful as a stepping stone for developing the string theory in the case of $2 \mathrm{~d}$ Yang Mills theory [70, 72] — for a proposal connecting this string theory to AdS dynamics, see [73]. The recent focus on tensor models, through their connection to SYK models, is directed towards the emergence of conformal symmetry and holographic duality involving AdS spaces (see the references mentioned in the introduction). The story of gauge-string duality (see e.g. the review [2]) involving low dimensional non-critical string backgrounds formulated as $c \leq 1$ matter coupled to Liouville theory, co-existing with alternative formulations in terms of topological strings, intersection theory on moduli spaces, or collective field theory may conceivably have a counterpart for tensor models. The unifying role of algebras related to permutations, and permutation topological field theories, in organizing physical correlators for matrix as well as tensor models suggests that such a scenario is not 
unrealistic and worth exploring. The key role of the Wedderburn-Artin decomposition of algebras, which finds an interpretation in open-closed topological string theory $[55,56,75]$ - with open strings associated to generic elements of the algebra and central elements associated with closed strings - suggests that finding an interpretation of these openclosed relations in the alternative holographic formulations of the string theory would be an interesting goal.

The $S_{n}$-TFT2 picture also gives a geometrical picture for correlators in the Fourier basis. Take for example the correlator (5.5) that is:

$$
\left\langle\mathcal{O}_{R_{1}, R_{2}, R_{3}}\right\rangle=n ! \mathrm{C}\left(R_{1}, R_{2}, R_{3}\right) \prod_{i=1}^{3} \frac{\operatorname{Dim}_{N}\left(R_{i}\right)}{d\left(R_{i}\right)}
$$

Consider the partition function of $S_{n}$ TFT2 on the 2-complex shown in figure 4

$$
\begin{aligned}
Z\left(\sigma_{1}, \sigma_{2}, \sigma_{3}\right) & =\sum_{\sigma_{0}, \gamma_{1}, \gamma_{2}, \gamma_{3} \in S_{n}} \delta\left(\sigma_{0} \gamma_{1} \sigma_{1} \gamma_{1}^{-1}\right) \delta\left(\sigma_{0} \gamma_{2} \sigma_{2} \gamma_{2}^{-1}\right) \delta\left(\sigma_{0} \gamma_{3} \sigma_{3} \gamma_{3}^{-1}\right) \\
& =n ! \sum_{\sigma_{1}, \sigma_{2}, \sigma_{3}, \gamma_{1}, \gamma_{2}} \delta\left(\sigma_{1} \gamma_{1} \sigma_{2} \gamma_{1}^{-1}\right) \delta\left(\sigma_{1} \gamma_{2} \sigma_{3} \gamma_{2}^{-1}\right)
\end{aligned}
$$

Considering the central correlators in the Fourier basis

$$
Z\left(P_{R_{1}}, P_{R_{2}}, P_{R_{3}}\right)=n ! \mathrm{C}\left(R_{1}, R_{2}, R_{3}\right)
$$

Inserting the central elements $\Omega=\sum_{\sigma \in S_{n}} N^{\mathbf{c}(\sigma)} \sigma$ we have

$$
Z\left(\frac{N^{n}}{n !} \Omega P_{R_{1}}, \frac{N^{n}}{n !} \Omega P_{R_{2}}, \frac{N^{n}}{n !} \Omega P_{R_{3}}\right)=n ! \frac{\operatorname{Dim}_{N}\left(R_{1}\right)}{d\left(R_{1}\right)} \frac{\operatorname{Dim}_{N}\left(R_{2}\right)}{d\left(R_{2}\right)} \frac{\operatorname{Dim}_{N}\left(R_{3}\right)}{d\left(R_{3}\right)} \mathrm{C}\left(R_{1}, R_{2}, R_{3}\right)
$$

Similarly, for $d=4$,

$$
Z\left(P_{R_{1}}, P_{R_{2}}, P_{R_{3}}, P_{R_{4}}\right)=n ! \mathrm{C}\left(R_{1}, R_{2}, R_{3}, R_{4}\right)
$$

and

$$
\begin{aligned}
& Z\left(\frac{N^{n}}{n !} \Omega P_{R_{1}}, \frac{N^{n}}{n !} \Omega P_{R_{2}}, \frac{N^{n}}{n !} \Omega P_{R_{3}}, \frac{N^{n}}{n !} \Omega P_{R_{4}}\right) \\
& =n ! \frac{\operatorname{Dim}_{N}\left(R_{1}\right)}{d\left(R_{1}\right)} \frac{\operatorname{Dim}_{N}\left(R_{2}\right)}{d\left(R_{2}\right)} \frac{\operatorname{Dim}_{N}\left(R_{3}\right)}{d\left(R_{3}\right)} \frac{\operatorname{Dim}_{N}\left(R_{4}\right)}{d\left(R_{4}\right)} \mathrm{C}\left(R_{1}, R_{2}, R_{3}, R_{4}\right)
\end{aligned}
$$

The 2-cellular complexes associated with the counting at $d=3$ and at $d=4$ have been given in figure 5 .

The different ways of factorizing this 4-point function corresponds to the different topologically equivalent ways of resolving the 4 copies of incident circles into successive 3 -fold incidences. This is illustrated in figure 6 . 


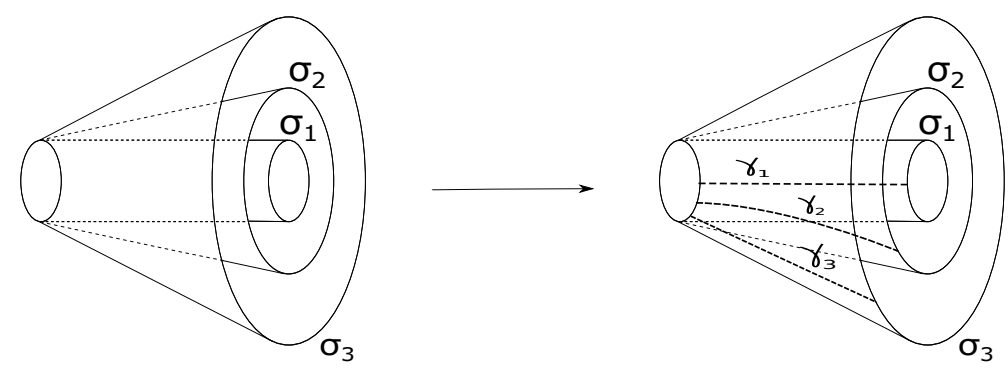

Figure 4. $S_{n}$ TFT2 for Kronecker coefficients: permutation basis.
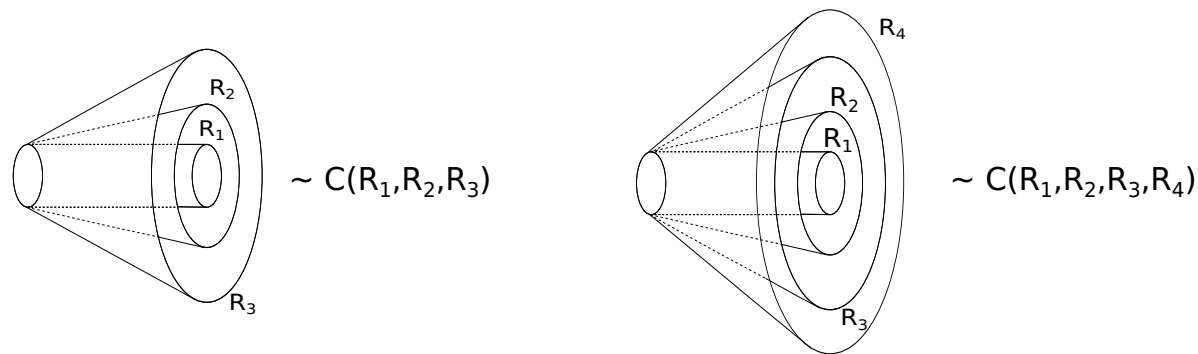

Figure 5. $S_{n}$ TFT2 for Kronecker coefficients: central in rep basis in $d=3$ (left) and $d=4$ (right).
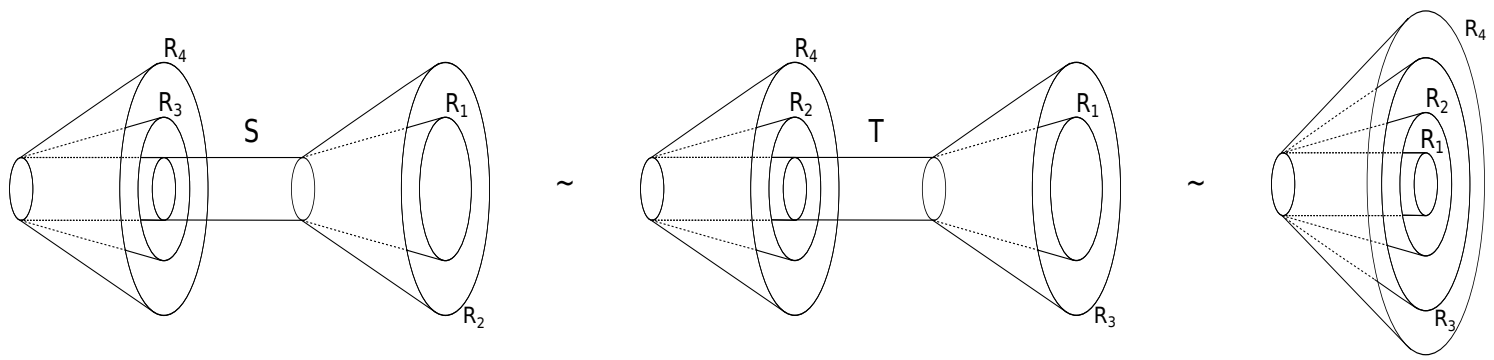

Figure 6. $S_{n}$ TFT2 for Kronecker coefficients: factorization equation (3.9) or (5.10).

\section{$7 \quad S_{3}$-color exchange symmetry}

The standard Gaussian integral over tensor fields is symmetric under exchange of the $d$ colors, and as such is expected to provide selection rules for correlators. This is also expected in any interacting model, which is obtained by adding to a Gaussian term an interaction which is invariant under color-exchange. It is therefore natural to consider the implications of the $S_{d}$ permutation group symmetry for the correlators of the tensor model and for the algebra $\mathcal{K}(n)$, which has been shown to be intimately related to correlators in previous sections. Colored symmetric tensor model observables have been enumerated in [41] using group algebra techniques. Color-symmetric interactions have also played a distinguished role as interactions in renormalizable tensor field theories [16-21].

The counting of color-symmetric observables in [41] was done in terms of sums over the conjugacy classes of $S_{d}$. For each conjugacy class $p$ in $S_{d}$, we had expressions $S_{p}^{(d)}$ which were sums over partitions of $S_{n}$. These were themselves observed to have integrality 
properties. Explicit formulae for $S_{p}^{(d)}$ were given for $d$ up to 4, and they have been computed to high orders in OEIS [76].

In this section, we will give formulae in terms of character sums for $S_{p}^{(d)}(n)$ for general $d$ and $p \vdash d$. We will consider the decomposition of $\mathcal{K}(n, d)$ in terms of symmetry types of $S_{d}$, labelled by Young diagrams $Y$ of $S_{d}$. The integrality of these will be used to prove that the integrality of $S_{p}^{(d)}(n)$. The completely symmetric Young diagram $Y=[d] \equiv Y_{0}$ corresponds to the counting of color-symmetrized graphs.

Focusing on the case $d=3$, where we have 3 colors, we describe selection rules for the multiplication in $\mathcal{K}(n)$ which follow from color-exchange symmetry. We observe that the subspace $\mathcal{K}_{Y_{0}}(n)$ forms a sub-algebra of $\mathcal{K}(n)$. Like $\mathcal{K}(n)$ it inherits a non-degenerate pairing from $\mathbb{C}\left(S_{n}\right) \otimes \mathbb{C}\left(S_{n}\right)$. The WA theorem implies therefore that it has a decomposition as a direct sum of matrix algebras. This in turn implies that we should be able to write a formula for the dimension of $\mathcal{K}_{Y_{0}}(n)$ as a sum of squares. We find such a formula and explain how to construct a basis for $\mathcal{K}_{Y_{0}}(n)$ which matches the counting. We give group theoretic formulae for the dimensions of the other $S_{3}$ invariant subspaces of $\mathcal{K}(n)$, namely $\mathcal{K}_{Y_{1}}(n), \mathcal{K}_{Y_{2}}(n)$ where $Y_{1}=[2,1], Y_{2}=[3]$ are the Young diagrams for mixed symmetry and for the antisymmetric representation of $S_{3}$.

\subsection{Counting observables in the rank $d=3$ case}

Color-symmetrised graphs are defined [41] by imposing an equivalence under $S_{3}$ permutations of the permutation triples describing the graph

$$
\left(\sigma_{1}, \sigma_{2}, \sigma_{3}\right) \sim\left(\sigma_{2}, \sigma_{1}, \sigma_{3}\right) \sim\left(\sigma_{1}, \sigma_{3}, \sigma_{2}\right) \sim \ldots
$$

These $S_{3}$ permutations commute with the diagonal left $S_{n}$ action and the diagonal right multiplication, which are used to get colored graphs from the permutation triples. Elements of $\left(\mathbb{C}\left(S_{n}\right)\right)^{\otimes 3}$ invariant under this $S_{3}$ action are

$$
\left[\sigma_{1} \sigma_{2} \sigma_{3}\right]:=\sum_{\alpha \in S_{3}} \sigma_{\alpha(1)} \otimes \sigma_{\alpha(2)} \otimes \sigma_{\alpha(3)} \in \mathbb{C}\left(S_{n}\right)^{\otimes 3}
$$

The left and right $S_{n}$ equivalences are imposed on these $S_{3}$ symmetric triples

$$
\left[\sigma_{1} \sigma_{2} \sigma_{3}\right] \sim\left[\gamma_{1}^{\otimes 3}\right]\left[\sigma_{1} \sigma_{2} \sigma_{3}\right]\left[\gamma_{2}^{\otimes 3}\right]=\sum_{\alpha \in S_{3}} \gamma_{1} \sigma_{\alpha(1)} \gamma_{2} \otimes \gamma_{1} \sigma_{\alpha(2)} \gamma_{2} \otimes \gamma_{1} \sigma_{\alpha(3)} \gamma_{2}
$$

The color-symmetric subspace of $\mathcal{K}(n)$, denoted $\mathcal{K}_{Y_{0}}(n)$ has a dimension given by

$$
\begin{aligned}
\operatorname{dim}\left(\mathcal{K}_{Y_{0}}(n)\right)= & \frac{1}{6 n !} \sum_{\gamma \in S_{n}} \sum_{\sigma_{2}, \sigma_{3} \in S_{n}} \delta\left(\gamma^{-1} \sigma_{2} \gamma \sigma_{2}^{-1}\right) \delta\left(\gamma^{-1} \sigma_{3} \gamma \sigma_{3}^{-1}\right) \\
& +\frac{1}{2 n !} \sum_{\gamma \in S_{n}} \sum_{\sigma \in S_{n}} \delta\left(\gamma^{2} \sigma \gamma^{-2} \sigma^{-1}\right)+\frac{1}{3 n !} \sum_{\gamma, \sigma \in S_{n}} \delta\left(\gamma^{3} \sigma^{3}\right) \\
= & \frac{1}{6 n !} \sum_{p \vdash n} \operatorname{Sym}(p)+\frac{1}{2 n !} \sum_{\gamma \in S_{n}} \sum_{\sigma \in S_{n}} \delta\left(\gamma^{2} \sigma \gamma^{-2} \sigma^{-1}\right)+\frac{1}{3 n !} \sum_{\gamma, \sigma \in S_{n}} \delta\left(\gamma^{3} \sigma^{3}\right) \\
= & \frac{1}{6} S_{\left[1^{3}\right]}^{(3)}(n)+\frac{1}{2} S_{[2,1]}^{(3)}(n)+\frac{1}{3} S_{[3]}^{(3)}(n)
\end{aligned}
$$


This was denoted $Z_{3 ; \text { cs }}(n)$ in [41]. Since the actions of $S_{3}$ commutes with $S_{n} \times S_{n}$, we in fact have an action of $S_{3} \times S_{n} \times S_{n}$ and we can equally first apply the $S_{n} \times S_{n}$ equivalence in $\mathbb{C}\left(S_{n}\right) \otimes \mathbb{C}\left(S_{n}\right) \otimes \mathbb{C}\left(S_{n}\right)$ to get $\mathcal{K}(n)$ and subsequently project to the invariants of the $S_{3}$ action. Following the steps in [41] (see equation (59) and following there) we see that the three terms can be written as

$$
S_{\left[1^{3}\right]}^{(3)}=\operatorname{tr}_{\mathcal{K}(n)}((1)(2)(3)), \quad S_{[2,1]}^{(3)}=\operatorname{tr}_{\mathcal{K}(n)}((12)), \quad S_{[3]}^{(3)}=\operatorname{tr}_{\mathcal{K}(n)}((123))
$$

where $\operatorname{tr}_{\mathcal{K}(n)}(\cdot)$ is a trace over the vector space $\mathcal{K}(n)$. We thus have

$$
\operatorname{dim}\left(\mathcal{K}_{Y_{0}}(n)\right)=\frac{1}{6} \operatorname{tr}_{\mathcal{K}(n)}((1)(2)(3))+\frac{1}{3} \operatorname{tr}_{\mathcal{K}(n)}((12))+\frac{1}{2} \operatorname{tr}_{\mathcal{K}(n)}((123))
$$

The Burnside lemma calculation in [41] can be regarded as the application of the normalized projector for $S_{n} \times S_{n} \times S_{3}$ acting on $\mathbb{C}\left(S_{n}\right) \otimes \mathbb{C}\left(S_{n}\right) \otimes \mathbb{C}\left(S_{n}\right)$ followed by taking the trace. This is because under inner product on $\mathbb{C}\left(S_{n}\right)$ given by the delta function, the permutations form an orthonormal basis.

\subsection{The algebra $\mathcal{K}_{Y_{0}}(n)$}

We define the algebra $\mathcal{K}_{Y_{0}}(n)$ the left and right invariant and colored symmetric sub-algebra of $\mathbb{C}\left(S_{n}\right)^{\otimes 3}$ as

$$
\mathcal{K}_{\mathrm{cs}}(n)=\operatorname{Span}_{\mathbb{C}}\left\{\sum_{\gamma_{1}, \gamma_{2}, \gamma_{3} \in S_{n}, \tau \in S_{3}} \gamma_{1} \sigma_{\tau(1)} \gamma_{2} \otimes \gamma_{1} \sigma_{\tau(2)} \gamma_{2} \otimes \gamma_{1} \sigma_{\tau(3)} \gamma_{2}, \sigma_{1}, \sigma_{2}, \sigma_{3} \in S_{n}\right\}
$$

It can be checked that the product of two basis elements of $\mathcal{K}_{\mathrm{cs}}(n), A=\sum_{\tau \in S_{3}, \gamma_{i} \in S_{n}}$ $\left(\gamma_{1} \sigma_{\tau(1)} \gamma_{2} \otimes \gamma_{1} \sigma_{\tau(2)} \gamma_{2} \otimes \gamma_{1} \sigma_{\tau(3)} \gamma_{2}\right)$ and $B=\sum_{\tau^{\prime} \in S_{3}, \gamma_{i}^{\prime} \in S_{n}} \gamma_{1}^{\prime} \sigma_{\tau^{\prime}(1)} \gamma_{2}^{\prime} \otimes \gamma_{1}^{\prime} \sigma_{\tau^{\prime}(2)} \gamma_{2}^{\prime} \otimes \gamma_{1}^{\prime} \sigma_{\tau^{\prime}(3)} \gamma_{2}^{\prime}$ belongs to $\mathcal{K}_{\mathrm{cs}}(n)$. We have

$A B$

$$
\begin{aligned}
& =\sum_{\tau, \tau^{\prime} \in S_{3}} \sum_{\gamma_{i}, \gamma_{i}^{\prime} \in S_{n}}\left(\gamma_{1} \sigma_{\tau(1)} \gamma_{2} \otimes \gamma_{1} \sigma_{\tau(2)} \gamma_{2} \otimes \gamma_{1} \sigma_{\tau(3)} \gamma_{2}\right)\left(\gamma_{1}^{\prime} \sigma_{\tau^{\prime}(1)} \gamma_{2}^{\prime} \otimes \gamma_{1}^{\prime} \sigma_{\tau^{\prime}(2)} \gamma_{2}^{\prime} \otimes \gamma_{1}^{\prime} \sigma_{\tau^{\prime}(3)} \gamma_{2}^{\prime}\right) \\
& =n ! \sum_{\tau^{\prime}, \gamma_{2} \in S_{3}}\left\{\sum_{\tau \in S_{3}} \sum_{\gamma_{1}, \gamma_{2}^{\prime} \in S_{n}} \gamma_{1}\left(\sigma_{\tau(1)} \gamma_{2} \sigma_{\tau^{\prime}(1)}\right) \gamma_{2}^{\prime} \otimes \gamma_{1}\left(\sigma_{\tau(2)} \gamma_{2} \sigma_{\tau^{\prime}(2)}\right) \gamma_{2}^{\prime} \otimes \gamma_{1}\left(\sigma_{\tau(3)} \gamma_{2} \sigma_{\tau^{\prime}(3)}\right) \gamma_{2}^{\prime}\right\}
\end{aligned}
$$

where we successively used $\gamma_{2} \gamma_{1}^{\prime} \rightarrow \gamma_{2}, \tau^{\prime} \rightarrow \tau^{\prime} \tau^{-1}$. Then, the product $A B$ is a sum of basis elements of $\mathcal{K}_{\mathrm{cs}}(n)$ hence belongs to $\mathcal{K}_{\mathrm{cs}}(n)$.

\subsection{Decomposition of $\mathcal{K}(n)$ into representations of $S_{3}$}

The group algebra $\mathbb{C}\left(S_{n}\right) \otimes \mathbb{C}\left(S_{n}\right) \otimes \mathbb{C}\left(S_{n}\right)$ is a representation of $S_{n} \times S_{n} \times S_{3}$. One $S_{n}$ acts on the diagonally on the left, the other acts diagonally on the right, and the $S_{3}$ permutes the three factors. These three actions commute. Once we have projected to the invariant subspace of $S_{n} \times S_{n}$ to get $\mathcal{K}(n)$ we still have an $S_{3}$ action. We can decompose $\mathcal{K}(n)$ into 
subspaces which transform as irreducible representations of $S_{3}$. We have a decomposition of $\mathcal{K}(n)$ into

$$
\mathcal{K}(n)=\mathcal{K}_{Y_{0}}(n) \oplus \mathcal{K}_{Y_{1}}(n) \oplus \mathcal{K}_{Y_{2}}(n)
$$

$Y_{0}=[3]$ is the one-dimensional trivial rep of $S_{3}$, corresponding to a Young diagram with a single row of length $3 . Y_{1}=[2,1]$ is the two-dimensional irrep of $S_{3} . Y_{2}=\left[1^{3}\right]$ is the anti-symmetric irrep of $S_{3}$. In other words the vector space of 3-colored graphs is a representation of $S_{3}$. This space can be decomposed into a direct sum of the trivial along with the $[2,1]$ and $\left[1^{3}\right]$ representations, which will all appear, generically with multiplicities.

Using the standard formula for the projector $P_{Y}$ in the group algebra of $S_{d}$

$$
P_{Y}=\frac{d(Y)}{d !} \sum_{\alpha \in S_{d}} \chi^{Y}(\alpha) \alpha
$$

and specializing to $d=3$, we can write

$$
\operatorname{dim}\left(\left(\mathcal{K}_{Y_{i}}(n)\right)\right)=\frac{1}{6} \sum_{\alpha \in S_{3}} d\left(Y_{i}\right) \chi^{Y_{i}}(\alpha) \operatorname{tr}_{\mathcal{K}(n)}(\alpha), \quad i=0,1,2
$$

We have

$$
\operatorname{dim}\left(\mathcal{K}_{Y_{0}}(n)\right)=\frac{1}{6} S_{\left[1^{3}\right]}^{(3)}(n)+\frac{1}{2} S_{[2,1]}^{(3)}(n)+\frac{1}{3} S_{[3]}^{(3)}(n)
$$

and

$$
\begin{aligned}
\operatorname{dim}\left(\mathcal{K}_{Y_{1}}(n)\right) & =\frac{2}{3} S_{\left[1^{3}\right]}^{(3)}(n)-\frac{2}{3} S_{[3]}^{(3)}(n) \\
\operatorname{dim}\left(\mathcal{K}_{Y_{2}}(n)\right) & =\frac{1}{6} S_{\left[1^{3}\right]}^{(3)}(n)-\frac{1}{2} S_{[2,1]}^{(3)}(n)+\frac{1}{3} S_{[3]}^{(3)}(n)
\end{aligned}
$$

Taking $a^{(0)}, a^{(1)}, a^{(2)}$ to belong to subspaces of $\mathcal{K}(n)$ labelled by the three Young diagrams $Y_{0}=\{3\}, Y_{1}=\{2,1\}, Y_{2}=\{1,1,1\}$ of $S_{3}$, we will have the following selection rules

$$
\begin{aligned}
& a^{(0)} \cdot a^{(i)} \in \mathcal{K}_{Y_{i}}(n) \\
& a^{(1)} \cdot a^{(1)} \in \mathcal{K}_{Y_{0}}(n) \oplus \mathcal{K}_{Y_{1}}(n) \oplus \mathcal{K}_{Y_{2}}(n) \\
& a^{(1)} \cdot a^{(2)} \in \mathcal{K}_{Y_{1}}(n) \\
& a^{(2)} \cdot a^{(2)} \in \mathcal{K}_{Y_{0}}(n)
\end{aligned}
$$

These follow from the corresponding tensor product decompositions of $S_{3}$ representations. From these equations we see that $\mathcal{K}_{Y_{0}}(n)$ is a sub-algebra of $\mathcal{K}(n) . \mathcal{K}_{Y_{1}}(n)$ and $\mathcal{K}_{Y_{2}}(n)$ are modules for the algebra $\mathcal{K}_{Y_{0}}(n) . \mathcal{K}_{Y_{0}} \oplus \mathcal{K}_{Y_{2}}$ is also a closed sub-algebra of $\mathcal{K}(n)$.

An interesting consequence of the above decomposition of $\mathcal{K}(n)$ is that we can use it to prove the integrality of the separate terms $S_{[13]}^{(3)}, S_{[2,1]}^{(3)}, S_{[3]}^{(3)}$. A similar argument can be made when we have $d$ colors instead of 3 . We will in fact present the argument in this generality. Take a vector space $V$, which is a representation of $S_{d}$. The multiplicity of an irrep labelled by Young diagram $Y$ (partition of $d$ ) is given by

$$
m_{V}^{Y}=\frac{1}{d !} \sum_{p \vdash d}\left|T_{p}\right| \chi^{Y}\left(\sigma_{p}\right) \operatorname{tr}_{V}\left(\sigma_{p}\right)=\sum_{p \vdash d} \frac{1}{|\operatorname{Sym}(p)|} \chi^{Y}\left(\sigma_{p}\right) \operatorname{tr}_{V}\left(\sigma_{p}\right)=\operatorname{tr}_{V}\left(\frac{P_{Y}}{d(Y)}\right)
$$


$\sigma_{p}$ is a permutation with cycle decomposition given by the partition $p$, i.e. some fixed permutation in the conjugacy class $T_{p}$. We will use $\widehat{T_{p}}$ to denote the sum of all the permutations in the conjugacy class $T_{p}$. The subspace of $V$ transforming in the irrep $Y$, denoted $V_{Y}$, has dimension

$$
\operatorname{dim} V_{Y}=\operatorname{tr}_{V}\left(P_{Y}\right)=m_{V}^{Y} d(Y)
$$

These $m_{V}^{Y}$ are natural numbers (zero or positive integers). The quantities we called $S_{p}^{(d)}$ in [41] are

$$
S_{p}^{(d)}=\operatorname{tr}_{V}\left(\sigma_{p}\right)=\frac{1}{\left|T_{p}\right|} \operatorname{tr}_{V}\left(\widehat{T_{p}}\right)
$$

for the case where $V=\mathcal{K}(n)$.

$\widehat{T_{p}}$ is a central element in the group algebra of $S_{n}$ and has an expansion in projectors

$$
\widehat{T_{p}}=\sum_{Y} \frac{\chi^{Y}\left(\widehat{T_{p}}\right)}{d(Y)} P_{Y}
$$

This can be seen by writing

$$
\widehat{T_{p}}=\sum_{Y^{\prime}} a_{Y^{\prime}} P_{Y^{\prime}}
$$

and then taking the trace in irrep $Y$ to find

$$
\chi^{Y}\left(\widehat{T_{p}}\right)=a_{Y} d(Y), \quad a_{Y}=\frac{\chi^{Y}\left(\widehat{T_{p}}\right)}{d(Y)}
$$

Now write

$$
\operatorname{tr}_{V}\left(\widehat{T_{p}}\right)=\sum_{R} \frac{\chi^{Y}\left(\widehat{T_{p}}\right)}{d(Y)} \operatorname{tr}_{V}\left(P_{Y}\right)=\sum_{R} \frac{\chi^{Y}\left(\widehat{T_{p}}\right)}{d(Y)} d(Y) m_{V}^{Y}=\sum_{R} \chi^{Y}\left(\widehat{T_{p}}\right) m_{V}^{Y}
$$

and observe

$$
\frac{1}{\left|T_{p}\right|} \operatorname{tr}_{V}\left(\widehat{T_{p}}\right)=\sum_{Y} \frac{\chi^{Y}\left(\widehat{T_{p}}\right)}{\left|T_{p}\right|} m_{V}^{Y}=\sum_{R} \frac{\chi^{Y}\left(\widehat{T_{p}}\right)}{\left|T_{p}\right|} \operatorname{tr}_{V}\left(\frac{P_{Y}}{d(Y)}\right)
$$

It turns out that, for symmetric groups, we know that the characters $\frac{\chi^{Y}\left(\widehat{T_{p}}\right)}{\left|T_{p}\right|}=\chi^{Y}\left(\sigma_{p}\right)$ are integers (this follows for example from the Murnaghan-Nakayama lemma for computing the characters). This proves that the $S_{p}^{(d)}-$ for all $d$ and $p-$ in our previous paper are integers. From the above equation it is not clear they are positive, but we also know from before that they are sums of delta functions. Combining these two facts, we conclude that they are indeed positive integers - as we found to high orders in [41] and in [76].

As a consistency check of the above (7.13) we can construct the sequence for the $Y_{1}$ sector $\frac{1}{d\left(Y_{1}\right)} \operatorname{dim}\left(\mathcal{K}_{Y_{1}}(n)\right)=\left(S_{\left[1^{3}\right]}^{(3)}-S_{[3]}^{(3)}\right) / 3$ - using the $S_{p}^{(3)}$ are found to be

$$
0,1,3,13,52,296,1850,14386,126082,1247479
$$

so indeed integral. Also for $\operatorname{dim}\left(\mathcal{K}_{Y_{2}}(n)\right)$, we find

$$
0,0,0,2,13,110,810,6796,61693,618880
$$

Again these are indeed integral as expected. 


\subsection{Color symmetrisation using Fourier basis}

We can better understand the connection between the $Q_{\tau_{1}, \tau_{2}}^{R_{1}, R_{2}, R_{3}}$ and the $S_{3}$ invariant subspaces, $\mathcal{K}_{Y}(n)$ by doing the color symmetrisation directly in the Fourier basis for $\mathbb{C}\left(S_{n}\right) \otimes$ $\mathbb{C}\left(S_{n}\right) \otimes \mathbb{C}\left(S_{n}\right)$

$$
Q_{i_{1}, j_{1}}^{R_{1}} \otimes Q_{i_{2}, j_{2}}^{R_{2}} \otimes Q_{i_{3}, j_{3}}^{R_{3}}
$$

Important properties (see appendix B.1) are

$$
Q_{i j}^{R}=\sqrt{\frac{d(R)}{n !}} \sum_{\sigma \in S_{n}} D_{i j}^{R}(\sigma) \sigma, \quad \tau Q_{i j}^{R}=\sum_{p} D_{p i}^{R}(\tau) Q_{p j}^{R}, \quad Q_{i j}^{R} \tau=\sum_{q} Q_{i q}^{R} D_{j q}^{R}(\tau)
$$

and the orthonormality property

$$
\delta\left(Q_{i j}^{R}, Q_{k l}^{S}\right)=\delta_{R, S} \delta_{i k} \delta_{j l}
$$

In order to project $(7.25)$ to $\mathcal{K}_{Y}(n)$ we apply the normalized projectors

$$
P^{\left(S_{3}\right)} P_{L}^{\left(S_{n}\right)} P_{R}^{\left(S_{n}\right)}=\frac{1}{6(n !)^{2}} \sum_{\sigma_{1} \in S_{n}} \sum_{\sigma_{2} \in S_{n}} \sum_{\alpha \in S_{3}} \alpha \rho_{L}\left(\sigma_{1}\right) \rho_{R}\left(\sigma_{2}\right)
$$

where $\rho_{L}\left(\sigma_{1}\right)$ indicates the left diagonal action, $\rho_{R}\left(\sigma_{2}\right)$ is the right diagonal action and $\alpha$ acts by swapping the tensor slots. We will calculate

$$
\operatorname{dim}\left(\mathcal{K}_{Y_{0}}(n)\right)=\operatorname{tr}_{\mathbb{C}\left(S_{n}\right)^{\otimes 3}}\left(P^{\left(S_{3}\right)} P_{L}^{\left(S_{n}\right)} P_{R}^{\left(S_{n}\right)}\right)=\operatorname{tr}_{\mathcal{K}(n)}\left(P^{\left(S_{3}\right)}\right)
$$

We have

$$
\begin{aligned}
& \sum_{\sigma_{1} \in S_{n}} \sum_{\sigma_{2} \in S_{n}} \sum_{\alpha \in S_{3}} \alpha \rho_{L}\left(\sigma_{1}\right) \rho_{R}\left(\sigma_{2}\right) Q_{i_{1}, j_{1}}^{R_{1}} \otimes Q_{i_{2}, j_{2}}^{R_{2}} \otimes Q_{i_{3}, j_{3}}^{R_{3}} \\
& =\sum_{\sigma_{1} \in S_{n}} \sum_{\sigma_{2} \in S_{n}} \sum_{\alpha \in S_{3}} \sum_{p_{l}, q_{l}} D_{p_{1} i_{1}}^{R_{1}}\left(\sigma_{1}\right) D_{p_{2} i_{2}}^{R_{2}}\left(\sigma_{1}\right) D_{p_{3} i_{3}}^{R_{3}}\left(\sigma_{1}\right) \\
& \quad D_{j_{1} q_{1}}^{R_{1}}\left(\sigma_{2}\right) D_{j_{2} q_{2}}^{R_{2}}\left(\sigma_{2}\right) D_{j_{3} q_{3}}^{R_{3}}\left(\sigma_{2}\right) \\
& \quad Q_{p_{\alpha(1)}, q_{\alpha(1)}}^{R_{\alpha(1)}} \otimes Q_{p_{\alpha(2)}, q_{\alpha(2)}}^{R_{\alpha(2)}} \otimes Q_{p_{\alpha(3)}, q_{\alpha(3)}}^{R_{\alpha(3)}}
\end{aligned}
$$

To compute the trace, pair this with $Q_{i_{1}, j_{1}}^{R_{1}} \otimes Q_{i_{2}, j_{2}}^{R_{2}} \otimes Q_{i_{3}, j_{3}}^{R_{3}}$ and sum over $R_{l}, i_{l}, j_{l}$ giving

$$
\begin{aligned}
& \sum \sum_{R_{l}} \sum_{\sigma_{l} \in S_{n}} \sum_{\alpha \in S_{3}} \sum_{i_{l}, j_{l}, p_{l}, q_{l}} D_{p_{1} i_{1}}^{R_{1}}\left(\sigma_{1}\right) D_{p_{2} i_{2}}^{R_{2}}\left(\sigma_{1}\right) D_{p_{3} i_{3}}^{R_{3}}\left(\sigma_{1}\right) \\
& D_{j_{1} q_{1}}^{R_{1}}\left(\sigma_{2}\right) D_{j_{2} q_{2}}^{R_{2}}\left(\sigma_{2}\right) D_{j_{3} q_{3}}^{R_{3}}\left(\sigma_{2}\right) \\
& \delta^{\left.R_{1}, R_{\alpha(1)}\right)} \delta_{i_{1}, p_{\alpha(1)}} \delta_{j_{1}, q_{\alpha(1)}} \delta^{R_{2}, R_{\alpha(2)}} \delta_{\left.i_{2}, p_{\alpha(2)}\right)} \delta_{j_{2}, q_{\alpha(2)}} \delta^{R_{3}, R_{\alpha(3)}} \delta_{i_{3}, p_{\alpha(3)}} \delta_{j_{3}, q_{\alpha(3)}} \\
= & \sum_{R_{1}, R_{2}, R_{3}} \sum_{\sigma_{1} \in S_{n}} \sum_{\sigma_{2} \in S_{n}} \sum_{\alpha \in S_{3}} \sum_{i_{l}, j_{l}} D_{i_{\alpha(1) i_{1}}}^{R_{1}}\left(\sigma_{1}\right) D_{i_{\alpha(2) i_{2}}}^{R_{2}}\left(\sigma_{1}\right) D_{i_{\alpha(3) i_{3}}}^{R_{3}}\left(\sigma_{1}\right) \\
& D_{j_{1} j_{\alpha(1)}}^{R_{1}}\left(\sigma_{2}\right) D_{j_{2} j_{\alpha(2)}}^{R_{2}}\left(\sigma_{2}\right) D_{j_{3} j_{\alpha(3)}}^{R_{3}}\left(\sigma_{2}\right) \delta^{R_{1}, R_{\alpha(1)}} \delta^{R_{2}, R_{\alpha(2)}} \delta^{R_{3}, R_{\alpha(3)}}
\end{aligned}
$$


There are three types of terms. If $\alpha$ is the identity then we have

$$
\begin{aligned}
& \frac{1}{6(n !)^{2}} \sum_{\sigma_{1}, \sigma_{2}} \chi^{R_{1}}\left(\sigma_{1}\right) \chi^{R_{2}}\left(\sigma_{1}\right) \chi^{R_{3}}\left(\sigma_{1}\right) \chi^{R_{1}}\left(\sigma_{2}\right) \chi^{R_{2}}\left(\sigma_{2}\right) \chi^{R_{3}}\left(\sigma_{2}\right) \delta^{R_{1}, R_{\alpha(1)}} \delta^{R_{2}, R_{\alpha(2)}} \delta^{R_{3}, R_{\alpha(3)}} \\
& \quad=\frac{1}{6} \sum_{R_{1}, R_{2}, R_{3}}\left(\mathrm{C}\left(R_{1}, R_{2}, R_{3}\right)\right)^{2}
\end{aligned}
$$

When $\alpha=(1,2)$ we get

$$
\begin{aligned}
& \frac{1}{6(n !)^{2}} \sum_{R_{1}, R_{3}} \sum_{\sigma_{1}, \sigma_{2}} \chi^{R_{1}}\left(\sigma_{1}^{2}\right) \chi^{R_{3}}\left(\sigma_{1}\right) \chi^{R_{1}}\left(\sigma_{2}^{2}\right) \chi^{R_{3}}\left(\sigma_{2}\right) \\
& =\frac{1}{6(n !)^{2}} \sum_{R_{1}} \sum_{\sigma_{1}, \sigma_{2}} \chi^{R_{1}}\left(\sigma_{1}^{2}\right) \chi^{R_{1}}\left(\sigma_{2}^{2}\right) \sum_{\gamma} \delta\left(\sigma_{1} \gamma \sigma_{2}^{-1} \gamma^{-1}\right) \\
& =\frac{1}{6(n !)} \sum_{R_{1}} \sum_{\sigma_{1}} \chi^{R_{1}}\left(\sigma_{1}^{2}\right) \chi^{R_{1}}\left(\sigma_{1}^{2}\right)
\end{aligned}
$$

The other permutations in the same conjugacy class give the same factor. So we get

$$
\frac{1}{2(n !)} \sum_{R} \sum_{\sigma} \chi^{R}\left(\sigma^{2}\right) \chi^{R}\left(\sigma^{2}\right)
$$

If $\alpha$ has cycle structure [3], then we have

$$
\frac{1}{3(n !)^{2}} \sum_{R} \sum_{\sigma_{1}, \sigma_{2}} \chi^{R}\left(\sigma_{1}^{3}\right) \chi^{R}\left(\sigma_{2}^{3}\right)
$$

So we recover the counting we previously had, from working with the delta functions over the group algebra.

These three expressions above

$$
\begin{aligned}
S_{p=\left[1^{3}\right]}^{(3)}(n) & =\sum_{R_{1}, R_{2}, R_{3}}\left(\mathrm{C}\left(R_{1}, R_{2}, R_{3}\right)\right)^{2} \\
S_{p=[2,1]}^{(3)}(n) & =\frac{1}{n !} \sum_{R} \sum_{\sigma} \chi^{R}\left(\sigma^{2}\right) \chi^{R}\left(\sigma^{2}\right) \\
S_{p=[3]}^{(3)}(n) & =\frac{1}{(n !)^{2}} \sum_{R} \sum_{\sigma_{1}, \sigma_{2}} \chi^{R}\left(\sigma_{1}^{3}\right) \chi^{R}\left(\sigma_{2}^{3}\right)
\end{aligned}
$$

are directly related to the delta functions derived in [41] by starting from Burnside lemma. The advantage of the present method is that the generalization to general $S_{p}^{(d)}(n)$ is easily done. We will describe this generalization in section 7.6 of these formulae in terms of character sums.

\subsubsection{Subspace of $\mathcal{K}(n)$ with $R_{1,2,3}$ all different}

Fixing three different representations of $S_{n}$, let us call them $\{R, S, T\}$, there are subspaces of $\mathcal{K}_{Y_{0}}(n), \mathcal{K}_{Y_{1}}(n), \mathcal{K}_{Y_{2}}(n)$ which come from the six different assignments of the list $\left[R_{1}, R_{2}, R_{3}\right]$ 
from the set $\{R, S, T\}$. From the equations above we see that in this case, we only get contributions from $\alpha=(1)(2)(3)$. Using (7.31) we find

$$
\operatorname{dim}\left(\mathcal{K}_{Y_{0}}^{(R, S, T)}(n)\right)=(\mathrm{C}(R, S, T))^{2}
$$

for the color-symmetrized subspace and for the subspaces transforming according to the other irreps of $S_{3}$

$$
\begin{aligned}
& \operatorname{dim}\left(\mathcal{K}_{Y_{1}}^{(R, S, T)}(n)\right)=2(\mathrm{C}(R, S, T))^{2} \\
& \operatorname{dim}\left(\mathcal{K}_{Y_{2}}^{(R, S, T)}(n)\right)=(\mathrm{C}(R, S, T))^{2}
\end{aligned}
$$

Focusing on $\mathcal{K}_{Y_{0}}^{(R, S, T)}(n)$, which forms an algebra of $\mathcal{K}(n)$, we can write a basis for this algebra as

$$
\sum_{i_{1}, i_{2}, i_{3}, j_{1}, j_{2}, j_{3}} \sum_{\alpha \in S_{3}} C_{i_{1}, i_{2} ; i_{3}}^{R, S T \tau_{1}} C_{i_{1}, i_{2} ; i_{3}}^{R, S ; T, \tau_{2}} Q_{i_{\alpha(1)}, j_{\alpha(1)}}^{\alpha(R)} \otimes Q_{i_{\alpha(2)}, j_{\alpha(2)}}^{\alpha(S)} \otimes Q_{i_{\alpha(3)}, j_{\alpha(3)}}^{\alpha(T)}
$$

Since $\tau_{1}, \tau_{2}$ run over a range of $\mathrm{C}(R, S, T)$ the counting of these basis elements agrees precisely with the dimension of this subspace.

\subsubsection{Subspace of $\mathcal{K}(n)$ where two $S_{n}$ irreps are equal and different from third}

Consider the subspace of $\mathcal{K}(n)$ where two of the $R_{1}, R_{2}, R_{3}$ are equal to $R$ and a third is equal to $S$. The $\alpha=()$ term will now contribute $3 / 6(\mathrm{C}(R, R, S))^{2}=1 / 2(\mathrm{C}(R, R, S))^{2}$. The factor of 3 comes from the choice of which of the $R_{i}$ is equal to $S$.

Consider for concreteness the term (7.33), for case where $R_{1}=R_{2}=R, R_{3}=S$. We will re-write this in a number of ways.

Let us return to $S_{[2,1]}$

$$
S_{[2,1]}(n)=\frac{1}{(n !)^{2}} \sum_{R, S} \sum_{\sigma_{1}, \sigma_{2}} \chi^{R}\left(\sigma_{1}^{2}\right) \chi^{S}\left(\sigma_{1}\right) \chi^{R}\left(\sigma_{2}^{2}\right) \chi^{S}\left(\sigma_{2}\right)
$$

This sum contains cases where $R=S$ as well as $R \neq S$. Focusing on the $R \neq S$ case, define

$$
S_{[2,1]}^{(R, R, S)}(n)=\frac{1}{(n !)^{2}} \sum_{\sigma_{1}, \sigma_{2}} \chi^{R}\left(\sigma_{1}^{2}\right) \chi^{S}\left(\sigma_{1}\right) \chi^{R}\left(\sigma_{2}^{2}\right) \chi^{S}\left(\sigma_{2}\right)
$$

The multiplicity of $S$ in the symmetric part of $V_{R} \otimes V_{R}$ is

$$
\begin{aligned}
\operatorname{Mult}\left(\operatorname{Sym}^{2}\left(V_{R}\right), V_{S}\right) & =\frac{1}{(n !)} \sum_{\sigma} \chi^{S}(\sigma) \operatorname{tr}_{R \otimes R}\left((\sigma \otimes \sigma) \frac{1+(12)}{2}\right) \\
& =\frac{1}{2 n !} \sum_{\sigma} \chi^{S}(\sigma)\left(\chi^{R}\left(\sigma^{2}\right)+\chi^{R}(\sigma) \chi^{R}(\sigma)\right) \\
& =\frac{1}{2} \mathrm{C}(R, R, S)+\frac{1}{2 n !} \sum_{\sigma} \chi^{S}(\sigma) \chi^{R}\left(\sigma^{2}\right)
\end{aligned}
$$

The multiplicity of $S$ in the anti-symmetric part of $V_{R} \otimes V_{R}$ is

$$
\operatorname{Mult}\left(\Lambda^{2}\left(V_{R}\right), V_{S}\right)=\frac{1}{2} \mathrm{C}(R, R, S)-\frac{1}{2 n !} \sum_{\sigma} \chi^{S}(\sigma) \chi^{R}\left(\sigma^{2}\right)
$$


So we learn that

$$
\frac{1}{n !} \sum_{\sigma} \chi^{S}(\sigma) \chi^{R}\left(\sigma^{2}\right)=\operatorname{Mult}\left(\operatorname{Sym}^{2}\left(V_{R}\right), V_{S}\right)-\operatorname{Mult}\left(\Lambda^{2}\left(V_{R}\right), V_{S}\right)
$$

So we can re-write

$$
S_{[2,1]}^{(R, R, S)}=\left(\operatorname{Mult}\left(\operatorname{Sym}^{2}\left(V_{R}\right), V_{S}\right)-\operatorname{Mult}\left(\Lambda^{2}\left(V_{R}\right), V_{S}\right)\right)^{2}
$$

From the above of course

$$
\mathrm{C}(R, R, S)=\operatorname{Mult}\left(\operatorname{Sym}^{2}\left(V_{R}\right), V_{S}\right)+\operatorname{Mult}\left(\Lambda^{2}\left(V_{R}\right), V_{S}\right)
$$

The two terms which contribute to the dimension of $\mathcal{K}(n)$ when we restrict the list $\left[R_{1}, R_{2}, R_{3}\right]$ to take values in $\{R, R, S\}$ are

$$
\frac{1}{2} \mathrm{C}(R, R, S)^{2}+\frac{1}{2} \mathrm{C}(R, R, S)=\frac{1}{2} \mathrm{C}(R, R, S)(\mathrm{C}(R, R, S)+1)
$$

This shows that the restriction of $\mathcal{K}_{Y_{0}}(n)$ to the sector where two of the irreps are equal is integer.

Another very instructive way to write the dimension of this subspace of $\mathcal{K}_{Y_{0}}(n)$ is

$$
\begin{aligned}
\frac{1}{2} \mathrm{C} & (R, R, S)^{2}+\frac{1}{2}\left(\operatorname{Mult}\left(\operatorname{Sym}^{2}\left(V_{R}\right), V_{S}\right)-\operatorname{Mult}\left(\Lambda^{2}\left(V_{R}\right), V_{S}\right)\right)^{2} \\
= & \frac{1}{2}\left(\operatorname{Mult}\left(\operatorname{Sym}^{2}\left(V_{R}\right), V_{S}\right)+\operatorname{Mult}\left(\Lambda^{2}\left(V_{R}\right), V_{S}\right)\right)^{2} \\
& +\frac{1}{2}\left(\operatorname{Mult}\left(\operatorname{Sym}^{2}\left(V_{R}\right), V_{S}\right)-\operatorname{Mult}\left(\Lambda^{2}\left(V_{R}\right), V_{S}\right)\right)^{2} \\
= & \left(\operatorname{Mult}\left(\operatorname{Sym}^{2}\left(V_{R}\right), V_{S}\right)\right)^{2}+\left(\operatorname{Mult}\left(\Lambda^{2}\left(V_{R}\right), V_{S}\right)\right)^{2}
\end{aligned}
$$

This formula gives an expression for the dimension of the $(R, R, S)$ subspace of $\mathcal{K}_{Y_{0}}(n)$ as a sum of squares. That is, we have identified the WA blocks of the decomposition.

Based on this counting, we can write down the basis elements for $\mathcal{K}_{Y_{0}}(n)$ in the subspace where two of the $R_{i}$ are equal to $R$ and the third is $S$. We define Clebsch-Gordan coefficients

$$
\begin{aligned}
& C_{i_{1}, i_{2}, i_{3}}^{R, R, S ;[2], \tau_{1}} \\
& C_{i_{1}, i_{2}, i_{3}}^{\left.R, R, S ; 1^{2}\right], \tau_{2}}
\end{aligned}
$$

The first are the Clebsch-Gordan coefficients coupling $\operatorname{Sym}^{2}(R) \otimes S$ to the trivial representation. The second are the Clebsch-Gordan coefficients coupling $\Lambda^{2}(R) \otimes S$ to the trivial representation. The basis elements in $\mathcal{K}_{Y_{0}}(n)$ are of two types

$$
\begin{aligned}
& C_{i_{1}, i_{2}, i_{3}}^{R, R, S[2], \tau_{1}} C_{j_{1}, j_{2}, j_{3}}^{R, R, S ;[2], \tau_{2}} \times \\
& \left(Q_{i_{1}, j_{1}}^{R} \otimes Q_{i_{2}, j_{2}}^{R} \otimes Q_{i_{3}, j_{3}}^{S}+Q_{i_{1}, j_{1}}^{R} \otimes Q_{i_{3}, j_{3}}^{S} \otimes Q_{i_{2}, j_{2}}^{R}+Q_{i_{3}, j_{3}}^{S} \otimes Q_{i_{1}, j_{1}}^{R} \otimes Q_{i_{2}, j_{2}}^{R}\right)
\end{aligned}
$$

and

$$
\begin{aligned}
& C_{i_{1}, i_{2}, i_{3}}^{R, R,\left[^{2}\right], \tau_{1}} C_{j_{1}, j_{2}, j_{3}}^{\left.R, R ; 1^{2}\right], \tau_{2}} \times \\
& \left(Q_{i_{1}, j_{1}}^{R} \otimes Q_{i_{2}, j_{2}}^{R} \otimes Q_{i_{3}, j_{3}}^{S}+Q_{i_{1}, j_{1}}^{R} \otimes Q_{i_{3}, j_{3}}^{S} \otimes Q_{i_{2}, j_{2}}^{R}+Q_{i_{3}, j_{3}}^{S} \otimes Q_{i_{1}, j_{1}}^{R} \otimes Q_{i_{2}, j_{2}}^{R}\right)
\end{aligned}
$$

These agree with the correct counting in (7.48). Note that this is different from the naive guess of $\mathrm{C}(R, R, S)^{2}$ for this sector. 


\subsubsection{Subspace of $\mathcal{K}(n)$ with all equal $R_{1}=R_{2}=R_{3}=R$}

The above allows us to guess what will happen when we consider the subspace of $\mathcal{K}_{Y_{0}}(n)$ corresponding to $\{R, R, R\}$. We should be able to write the dimension of that as

$$
\left(\operatorname{Mult}\left(P_{[3]} V_{R}^{\otimes 3}, V_{0}\right)\right)^{2}+\left(\operatorname{Mult}\left(P_{[2,1]} V_{R}^{\otimes 3}, V_{0}\right)\right)^{2}+\left(\operatorname{Mult}\left(P_{\left[1^{3}\right]} V_{R}^{\otimes 3}, V_{0}\right)\right)^{2}
$$

The subscripts on the $P$ 's are Young diagrams with three boxes. Maybe we need a factor of 2 or 4 in front of the second term. This would be the WA decomposition of the $\mathcal{K}_{Y_{0}}(n)$ projected to the $(R, R, R)$ sector.

We now prove this. So we apply the projector

$$
\begin{aligned}
& \frac{1}{6(d !)^{2}} \sum_{\alpha \in S_{3}} \sum_{\sigma_{1}, \sigma_{2} \in S_{n}} \alpha \rho_{L}\left(\sigma_{1}\right) \rho_{R}\left(\sigma_{2}\right) Q_{i_{1}, j_{1}}^{R} \otimes Q_{i_{2} j_{2}}^{R} \otimes Q_{i_{3} j_{3}}^{R} \\
& =\frac{1}{6(d !)^{2}} \sum_{\alpha \in S_{3}} \sum_{\sigma_{1}, \sigma_{2} \in S_{n}} D_{p_{1} i_{1}}^{R}\left(\sigma_{1}\right) D_{p_{2} i_{2}}^{R}\left(\sigma_{1}\right) D_{p_{3} i_{3}}^{R}\left(\sigma_{1}\right) D_{j_{1} q_{1}}^{R}\left(\sigma_{2}\right) D_{j_{2} q_{2}}^{R}\left(\sigma_{2}\right) D_{j_{3} q_{3}}^{R}\left(\sigma_{2}\right) \\
& \quad \times Q_{p_{\alpha(1)}, q_{\alpha(1)}}^{R} \otimes Q_{p_{\alpha(2)} q_{\alpha(2)}}^{R} \otimes Q_{p_{\alpha(3)} q_{\alpha(3)}}^{R}
\end{aligned}
$$

Pair this with $Q_{i_{1}, j_{1}}^{R} \otimes Q_{i_{2} j_{2}}^{R} \otimes Q_{i_{3} j_{3}}^{R}$ and sum over $R, p_{l}$, and $q_{l}$ to find

$$
\begin{aligned}
& \frac{1}{6(d !)^{2}} \sum_{\alpha \in S_{3}, \sigma_{l} \in S_{n}} \sum_{p_{l}, q_{l}} D_{p_{1} p_{\alpha(1)}}^{R}\left(\sigma_{1}\right) D_{p_{2} p_{\alpha(2)}}^{R}\left(\sigma_{1}\right) D_{p_{3} p_{\alpha(3)}}^{R}\left(\sigma_{1}\right) D_{q_{\alpha(1)} q_{1}}^{R}\left(\sigma_{2}\right) D_{q_{\alpha(2)} q_{2}}^{R}\left(\sigma_{2}\right) D_{q_{\alpha(3)} q_{3}}^{R}\left(\sigma_{2}\right) \\
& =\sum_{\alpha} \sum_{\sigma_{1} \in S_{n}} \sum_{\sigma_{2} \in S_{n}} \operatorname{tr}_{R^{\otimes 3}}\left(\sigma_{1} \alpha\right) \operatorname{tr}_{R^{\otimes 3}}\left(\sigma_{2} \alpha\right)=\sum_{\alpha} \sum_{\sigma_{1} \in S_{n}} \sum_{\sigma_{2} \in S_{n}} \operatorname{tr}_{R^{\otimes 3} \otimes R^{\otimes 3}}\left(\sigma_{1} \otimes \sigma_{2}\right)(\alpha \otimes \alpha)
\end{aligned}
$$

This can be understood in terms of representation theory of $S_{n} \times S_{3}$ acting on $V_{R}^{\otimes 3}$.

$$
V_{R}^{\otimes 3}=\bigoplus_{\Lambda_{1} \vdash n} \bigoplus_{\Lambda_{2} \vdash 3} V_{\Lambda_{1}}^{\left(S_{n}\right)} \otimes V_{\Lambda_{2}}^{\left(S_{3}\right)} \otimes V_{R: \Lambda_{1}, \Lambda_{2}}
$$

We take two copies of these tensor products

$$
V_{R}^{\otimes 3} \otimes V_{R}^{\otimes 3}=\bigoplus_{\Lambda_{1}, \Lambda_{1}^{\prime} \vdash n} \bigoplus_{\Lambda_{2}, \Lambda_{2}^{\prime} \vdash 3} V_{\Lambda_{1}}^{\left(S_{n}\right)} \otimes V_{\Lambda_{2}}^{\left(S_{3}\right)} \otimes V_{R: \Lambda_{1}, \Lambda_{2}} \otimes V_{\Lambda_{1}^{\prime}}^{\left(S_{n}\right)} \otimes V_{\Lambda_{2}^{\prime}}^{\left(S_{3}\right)} \otimes V_{R: \Lambda_{1}^{\prime}, \Lambda_{2}^{\prime}}
$$

The sums over $\sigma_{1}, \sigma_{2} \in S_{n}$ project to $\Lambda_{1}=\Lambda_{1}^{\prime}=[n]$. We further need to restrict to the trivial of the diagonal $S_{3}$. This trivial rep occurs once inside $V_{\Lambda_{2}} \otimes V_{\Lambda_{2}^{\prime}}$ whenever $\Lambda_{2}=\Lambda_{2}^{\prime}$. The multiplicity is

$$
\sum_{\Lambda \vdash 3}\left(\operatorname{dim} V_{R:[n], \Lambda}\right)^{2}
$$

This gives the WA decomposition of $\mathcal{K}_{Y_{0}}(n)$ projected by overlapping the permutation triples with $P_{R} \otimes P_{R} \otimes P_{R}$.

Corresponding to the decomposition (7.55) there are Clebsch-Gordan coefficients for the change of basis from an orthogonal basis of states $\left|R, R, R ; i_{1}, i_{2}, i_{3}\right\rangle$ to another orthogonal basis of states $\left|\Lambda_{1}, \Lambda_{2}, \tau_{\Lambda_{1}, \Lambda_{2}} ; m_{\Lambda_{1}}, m_{\Lambda_{2}}\right\rangle$ as

$$
C_{i_{1}, i_{2}, i_{3} ; m_{\Lambda_{1}}, m_{\Lambda_{2}}}^{R, R, R ; \Lambda_{1}, \Lambda_{2}, \tau_{\Lambda_{1}, \Lambda_{2}}}=\left\langle\Lambda_{1}, \Lambda_{2}, \tau_{\Lambda_{1}, \Lambda_{2}} ; m_{\Lambda_{1}}, m_{\Lambda_{2}} \mid R, R, R ; i_{1}, i_{2}, i_{3}\right\rangle
$$


For the case $\Lambda_{1}=[n], \Lambda_{2}=\Lambda$, we have

$$
C_{i_{1}, i_{2}, i_{3} ; m_{\Lambda}}^{R, R, R ; n], \Lambda, \tau_{[n], \Lambda}}=\left\langle[n], \Lambda, \tau_{[n], \Lambda} ; m_{\Lambda} \mid R, R, R ; i_{1}, i_{2}, i_{3}\right\rangle
$$

The irrep $[n]$ is one-dimensional so has no corresponding state label.

The elements of the WA basis for $\mathcal{K}_{Y_{0}}(n)$ will be labelled by $\left(R, \Lambda, \tau_{[n], \Lambda}^{(l)}, \tau_{[n], \Lambda}^{(r)}\right)$ where $\tau_{[n], \Lambda}^{(l)}, \tau_{[n], \Lambda}^{(r)}$ label the multiplicity of $V_{[n]} \otimes V_{\Lambda}$ in the left and right $V_{R}^{\otimes 3}$ of (7.56). Using the Clebsch-Gordan coefficients (7.59) we can therefore write the WA basis

$$
Q^{R, \Lambda, \tau_{[n], \Lambda}^{(l)}, \tau_{[n], \Lambda}^{(r)}}=\sum_{i_{l}, j_{l}, m_{\Lambda}} C_{i_{1}, i_{2}, i_{3}}^{R, R, R ; \Lambda, m_{\Lambda}, \tau_{[n], \Lambda}^{(l)}} C_{j_{1}, j_{2}, j_{3}}^{R, R, R ; \Lambda, m_{\Lambda}, \tau_{[n], \Lambda}^{(r)}} Q_{i_{1} j_{1}}^{R} \otimes Q_{i_{2} j_{2}}^{R} \otimes Q_{i_{3} j_{3}}^{R}
$$

which matches the counting of states in this sector. Following steps similar to the ones for $Q^{R, S, T ; \tau_{1}, \tau_{2}}$ in the case of $\mathcal{K}(n)$, the orthogonality properties of these Clebsch-Gordan coefficients can be used to show that these form a basis of matrix units for $\mathcal{K}_{Y_{0}}(n)$ in the subspace corresponding to $R=S=T$.

A consequence of the above discussion (in particular equations (7.37), (7.48) and (7.57)) is that we can write the dimension of the algebra $\mathcal{K}_{Y_{0}}(n)$ as a sum of squares, corresponding to the WA decomposition

$$
\begin{aligned}
\operatorname{dim}\left(\mathcal{K}_{Y_{0}}(n)\right)= & \sum_{R \neq S \neq T}(\mathrm{C}(R, S, T))^{2}+\sum_{R \neq S}\left(\operatorname{Mult}\left(\operatorname{Sym}^{2}(R), S\right)\right)^{2}+\left(\operatorname{Mult}\left(\Lambda^{2}(R), S\right)\right)^{2} \\
& +\sum_{R} \sum_{\Lambda}\left(\operatorname{Mult}\left(R^{\otimes 3},[n] \otimes \Lambda\right)\right)^{2}
\end{aligned}
$$

This color-symmetrized analog of (3.6) is a key result of the present paper.

\subsubsection{More on $S_{[2,1]}^{(3)}(n)$ and the character table of $S_{n}$}

A useful fact is that for any irrep $R$ of $S_{n}$, the trivial appears in the symmetric part of $V_{R} \otimes V_{R}$ with multiplicity one. This is related to the reality of these representations (eq. 5.82 of [77]). It leads to the identify

$$
\frac{1}{n !} \sum_{\sigma} \operatorname{tr}_{R \otimes R}\left(\sigma \frac{(1+(12))}{2}\right)=\frac{1}{n !} \sum_{\sigma} \operatorname{tr}_{R \otimes R}(\sigma)
$$

The r.h.s. side counts the number of times the trivial representation appears in $R \otimes R$. The l.h.s. counts the number of times it appears in the symmetric part. This leads to

$$
\frac{1}{2 n !} \sum_{\sigma \in S_{n}}\left(\left(\chi^{R}(\sigma)\right)^{2}+\chi^{R}\left(\sigma^{2}\right)\right)=\frac{1}{n !} \sum_{\sigma \in S_{n}}\left(\chi^{R}(\sigma)\right)^{2}
$$

This in turn implies that

$$
\sum_{\sigma \in S_{n}}\left(\chi^{R}(\sigma)\right)^{2}=\sum_{\sigma \in S_{n}}\left(\chi^{R}\left(\sigma^{2}\right)\right)
$$


It also follows that

$$
\sum_{\sigma \in S_{n}} \delta\left(\tau^{-1} \sigma^{2}\right)=\sum_{S} \chi^{S}(\tau)
$$

The number of permutations which squares to $\tau$ can be written as a sum of characters in all irreps. To see this, use

$$
\begin{aligned}
\sum_{\sigma \in S_{n}} \delta\left(\tau^{-1} \sigma^{2}\right) & =\frac{1}{n !} \sum_{\sigma \in S_{n}} \sum_{S \vdash n} \chi^{S}(\tau) \chi^{S}\left(\sigma^{2}\right) \\
& =\frac{1}{n !} \sum_{\sigma \in S_{n}} \sum_{S \vdash n} \chi^{S}(\tau) \chi^{S}(\sigma) \chi^{S}(\sigma) \\
& =\sum_{S} \chi^{S}(\tau)
\end{aligned}
$$

We can also write

$$
\begin{aligned}
\frac{1}{n !} \sum_{R, \sigma} \chi^{R}\left(\sigma^{2}\right) \chi^{R}\left(\sigma^{2}\right) & =\frac{1}{n !} \sum_{R, \sigma, \tau} \chi^{R}(\tau) \chi^{R}(\tau) \delta\left(\tau^{-1} \sigma^{2}\right) \\
& =\frac{1}{n !} \sum_{R, S, \tau} \chi^{R}(\tau) \chi^{R}(\tau) \chi^{S}(\tau) \\
& =\sum_{R, S} \mathrm{C}(R, R, S)
\end{aligned}
$$

So the second contribution

$$
S_{[2,1]}=\sum_{R, S} \mathrm{C}(R, R, S)
$$

Also if we do the sum over $R$, we get

$$
S_{[2,1]}=\frac{1}{n !} \sum_{S} \sum_{\tau} \chi^{S}(\tau) \operatorname{Sym}(\tau)=\sum_{p} \sum_{S} \chi^{S}\left(\tau_{p}\right)
$$

We are summing over irreps $S$ and conjugacy classes. The weight is the character of a permutation in the specified conjugacy class, here denoted $\tau_{p}$ for conjugacy class specified by $p$. Indeed OEIS recognizes $S_{[2,1]}$ as the sum of entries of the character table of $S_{n}$. The refinement of $S_{[2,1]}$ parametrized by $S$ (where we drop the sum over $S$ )

$$
\sum_{p} \chi^{S}\left(\tau_{p}\right)
$$

is the subject of an open question posed by Stanley (problem 12 in [78]): to find a combinatoric construction which makes the positive integrality manifest. The positive integrality is manifest because

$$
\sum_{R} \mathrm{C}(R, R, S)=\sum_{p} \chi^{S}\left(\tau_{p}\right)
$$


(an identity that has been used above) but this is a representation theoretic argument, not a purely combinatoric one. Tensor invariants at large $N$ (or equivalently colored graphs) provide a combinatoric interpretation of the sum of squares of the Kronecker coefficients. It would be interesting to investigate whether refined consideration of colored graphs can provide an approach to this question of Stanley.

\section{5 $S_{3}$-refinement for $\mathcal{K}(n)$}

We have given above the dimension of the color-symmetrized subspace $\mathcal{K}_{Y_{0}}(n)$ as a sum over representation theoretic data. The expression is a sum of squares as expected from the WA decomposition. This shows that the representation theoretic construction perspective based on permutations and Fourier transforms naturally leads to the explicit form of the Matrix blocks of the WA decomposition. The expression is a sum over three types of terms, which we may describe as $(R, S, T)$ types involving three distinct Young diagrams, the $(R, R, S)$ which involves two distinct types and the $(R, R, R)$ which involves one type. Here we give the dimensions of

$$
\mathcal{K}_{Y}^{(R, S, T)}, \mathcal{K}_{Y}^{(R, R, S)}, \mathcal{K}_{Y}^{(R, R, R)}
$$

general Young diagram $Y$ of $S_{3}$.

For the $(R, S, T)$ sector, the answer is easy when we consider the restriction of the trace

$$
\operatorname{tr}_{\mathbb{C}\left(S_{n}\right) \otimes 3}\left(P_{Y} P_{L}^{S_{n}} P_{R}^{S_{n}}\right)=\operatorname{tr}_{\mathcal{K}(n)}\left(P_{Y}\right)
$$

to the subspace of $\mathbb{C}\left(S_{n}\right) \otimes \mathbb{C}\left(S_{n}\right) \otimes \mathbb{C}\left(S_{n}\right)$ to the Fourier basis states $Q_{i_{1}, j_{1}}^{R_{1}} \otimes Q_{i_{2}, j_{2}}^{R_{2}} \otimes Q_{i_{3}, j_{3}}^{R_{3}}$ where $R_{1}, R_{2}, R_{3}$ are all different and take values in the set $(R, S, T)$. There are 6 choices, which add up to $6 \mathrm{C}(R, S, T)^{2}$. In the expansion of $P_{Y}$ in terms of permutations only the identity permutation contributes and we have

$$
\mathcal{K}_{Y}^{(R, S, T)}=d(Y)^{2} \mathrm{C}(R, S, T)^{2}
$$

Hence we have

$$
\begin{aligned}
& \operatorname{dim} \mathcal{K}_{[3]}^{(R, S, T)}=\mathrm{C}(R, S, T)^{2} \\
& \operatorname{dim} \mathcal{K}_{[2,1]}^{(R, S, T)}=4 \mathrm{C}(R, S, T)^{2} \\
& \operatorname{dim} \mathcal{K}_{\left[1^{3}\right]}^{(R, S, T)}=\mathrm{C}(R, S, T)^{2}
\end{aligned}
$$

For the $(R, R, S)$ sector, we find

$$
\begin{aligned}
& \operatorname{dim}\left(\mathcal{K}_{Y=[3]}^{(R, R, S)}\right)=\left(\operatorname{Mult}\left(\operatorname{Sym}^{2}\left(V_{R}\right), V_{S}\right)\right)^{2}+\left(\operatorname{Mult}\left(\Lambda^{2}\left(V_{R}\right), V_{S}\right)\right)^{2} \\
& \operatorname{dim}\left(\mathcal{K}_{Y=[2,1]}^{(R, R, S)}\right)=2 \mathrm{C}(R, R, S)^{2} \\
& \operatorname{dim}\left(\mathcal{K}_{Y=\left[1^{3}\right]}^{(R, R, S)}\right)=2 \operatorname{Mult}\left(\operatorname{Sym}^{2}\left(V_{R}\right), V_{S}\right) \operatorname{Mult}\left(\Lambda^{2}\left(V_{R}\right), V_{S}\right)
\end{aligned}
$$

For the $(R, R, R)$ sector, we find

$$
\begin{aligned}
& \operatorname{dim}\left(\mathcal{K}_{Y}^{(R, R, R)}(n)\right) \\
& =\sum_{Y \vdash 3} d(Y) \mathrm{C}\left(Y_{1}, Y_{2}, Y\right) \operatorname{Mult}\left(V_{R}^{\otimes 3}, V_{\Lambda_{1}}^{\left(S_{3}\right)} \otimes V_{[n]}^{\left(S_{n}\right)}\right) \operatorname{Mult}\left(V_{R}^{\otimes 3}, V_{\Lambda_{2}}^{\left(S_{3}\right)} \otimes V_{[n]}^{\left(S_{n}\right)}\right)
\end{aligned}
$$


$\mathrm{C}\left(Y_{1}, Y_{2}, Y\right)$ is the Kronecker coefficient which counts the number of invariants of $S_{3}$ in the tensor product $Y_{1} \otimes Y_{2} \otimes Y$.

The derivation of these formulae proceeds by unravelling the equation (7.73) in each of these case. Some interesting consistency checks of these formulae can be easily given. We have the identity

$$
d(Y) \mathrm{C}\left(Y_{1}, Y_{2}, Y\right)=\sum_{Y} \sum_{\sigma} \frac{1}{d !} \chi^{Y_{1}}(\sigma) \chi^{Y_{2}}(\sigma) \chi^{Y}(\sigma)=d\left(Y_{1}\right) d\left(Y_{2}\right)
$$

Doing the sum over irreps $Y$ gives a delta function. For the $(R, R, R)$ case, therefore we have

$$
\begin{aligned}
& \sum_{Y} \operatorname{dim}\left(\mathcal{K}_{Y}^{(R, R, R)}(n)\right) \\
& \quad=\sum_{Y_{1}, Y_{2}} d\left(Y_{1}\right) d\left(Y_{2}\right) \operatorname{Mult}\left(V_{R}^{\otimes 3}, V_{Y_{1}}^{\left(S_{3}\right)} \otimes V_{[n]}^{S_{n}}\right) \operatorname{Mult}\left(V_{R}^{\otimes 3}, V_{Y_{2}}^{\left(S_{3}\right)} \otimes V_{[n]}^{\left(S_{n}\right)}\right) \\
& \quad=\mathrm{C}(R, R, R)^{2}
\end{aligned}
$$

Similarly, the reader can easily convince herself that

$$
\sum_{Y} \operatorname{dim}\left(\mathcal{K}_{Y}^{(R, R, S)}(n)\right)=3(\mathrm{C}(R, R, S))^{2}
$$

The 3 comes from the fact that when the ordered list of Young diagrams $\left[R_{1}, R_{2}, R_{3}\right]$ takes values from the set $\{R, R, S\}$, there are three possibilities.

An interesting consequence of the multiplication rule given in (7.14) is that $\mathcal{K}_{Y_{0}}(n) \oplus$ $\mathcal{K}_{Y_{2}}(n)$ is a closed associative algebra. It will inherit a non-degenerate bilinear form from the $\mathbb{C}\left(S_{n}\right) \otimes \mathbb{C}\left(S_{n}\right) \otimes \mathbb{C}\left(S_{n}\right)$ (or from $\mathbb{C}\left(S_{n}\right) \otimes \mathbb{C}\left(S_{n}\right)$ ) if we are working with the gauge-fixed formulation. So we expect that its dimension will be a sum of squares. The counting in terms of representations above automatically leads to such a sum of squares. For the $(R, S, T)$ subspace

$$
\operatorname{dim}\left(\mathcal{K}_{Y_{0}}^{(R, S, T)}\right)+\operatorname{dim}\left(\mathcal{K}_{Y_{1}}^{(R, S, T)}\right)=2(\mathrm{C}(R, S, T))^{2}
$$

For the $(R, R, S)$ subspace,

$$
\begin{aligned}
\operatorname{dim} & \mathcal{K}_{Y_{0}}^{(R, R, S)}+\operatorname{dim} \mathcal{K}_{Y_{2}}^{(R, R, S)} \\
= & \left(\operatorname{Mult}\left(\operatorname{Sym}^{2}\left(V_{R}\right), V_{S}\right)\right)^{2}+\left(\operatorname{Mult}\left(\Lambda^{2}\left(V_{R}\right), V_{S}\right)\right)^{2} \\
& +2 \operatorname{Mult}\left(\operatorname{Sym}^{2}\left(V_{R}\right), V_{S}\right) \operatorname{Mult}\left(\Lambda^{2}\left(V_{R}\right), V_{S}\right) \\
= & \left(\operatorname{Mult}\left(\operatorname{Sym}^{2}\left(V_{R}\right), V_{S}\right)+\operatorname{Mult}\left(\Lambda^{2}\left(V_{R}\right), V_{S}\right)\right)^{2} \\
= & (\mathrm{C}(R, R, S))^{2}
\end{aligned}
$$


For the $(R, R, R)$ subspace

$$
\begin{aligned}
& \left(\operatorname{Mult}\left(V_{R}^{\otimes 3}, V_{[3]}^{\left(S_{3}\right)} \otimes V_{[n]}^{\left(S_{n}\right)}\right)\right)^{2}+\left(\operatorname{Mult}\left(V_{R}^{\otimes 3}, V_{\left[1^{3}\right]}^{\left(S_{3}\right)} \otimes V_{[n]}^{\left(S_{n}\right)}\right)\right)^{2} \\
& \quad+4\left(\operatorname{Mult}\left(V_{R}^{\otimes 3}, V_{[2,1]}^{\left(S_{3}\right)} \otimes V_{[n]}^{\left(S_{n}\right)}\right)\right)^{2} \\
& \quad+2\left(\operatorname{Mult}\left(V_{R}^{\otimes 3}, V_{[3]}^{\left(S_{3}\right)} \otimes V_{[n]}^{\left(S_{n}\right)}\right)\right)\left(\operatorname{Mult}\left(V_{R}^{\otimes 3}, V_{\left[1^{3}\right]}^{\left(S_{3}\right)} \otimes V_{[n]}^{\left(S_{n}\right)}\right)\right) \\
& =\left(\operatorname{Mult}\left(V_{R}^{\otimes 3}, V_{[3]}^{\left(S_{3}\right)} \otimes V_{[n]}^{\left(S_{n}\right)}\right)\right)^{2}+\left(\operatorname{Mult}\left(V_{R}^{\otimes 3}, V_{\left[1_{3}^{3}\right]}^{\left(S_{3}\right)} \otimes V_{[n]}^{\left(S_{n}\right)}\right)\right)^{2} \\
& \quad+4\left(\operatorname{Mult}\left(V_{R}^{\otimes 3}, V_{[2,1]}^{\left(S_{3}\right)} \otimes V_{[n]}^{\left(S_{n}\right)}\right)\right)^{2}
\end{aligned}
$$

These counting formulae for $\operatorname{dim} \mathcal{K}_{Y}(n)$ in terms of representation theory data sets the stage for developing representation theoretic bases. Using the basic technique of using permutations to construct observables (2.7), these elements of $\mathcal{K}_{Y}(n)$ will be expected to give a refined orthonormal basis for the gauge-invariant observables, with good quantum numbers for the $S_{3}$ color-exchange, as we described earlier for $Y=Y_{0}$. This will be an interesting refinement of the results on orthogonal bases given earlier in section 5.3 and in $[38,59]$.

\subsection{Counting color-symmetrised tensor invariants for general $d$}

This way of approaching the calculation using Fourier transforms, presented for $d=3$ at the start of this section, allows us to generalize to any $d$. We get

Number of color symmetrised tensor invariants of rank $d=$

$$
\frac{1}{d !(n !)^{2}} \sum_{\alpha \in S_{d}} \sum_{R_{1}, \cdots, R_{d}} \sum_{\sigma_{1} \in S_{n}} \sum_{\sigma_{2} \in S_{n}} \prod_{a} \chi^{R^{(a)}}\left(\sigma_{1}^{l_{a}}\right) \chi^{R^{(a)}}\left(\sigma_{2}^{l_{a}}\right) \delta\left(R_{\alpha_{a}^{1}}, R_{\alpha_{a}^{2}}, \cdots, R_{\alpha_{a}^{l_{a}}}, R^{(a)}\right)
$$

Here the index $a$ runs over the cycles of the permutation $\alpha ; l_{a}$ is the length of the cycle. Each such cycle is of the form $\left(\alpha_{a}^{1}, \alpha_{a}^{2}, \cdots, \alpha_{a}^{l_{a}}\right)$ where the entries in the cycle are integers chosen from $\{1, \cdots d\}$. Such a cycle leads to delta functions enforcing $R_{\alpha_{a}^{1}}=R_{\alpha_{a}^{2}} \cdots=R_{\alpha_{a}^{l_{a}}}$, which leads to the definition

$$
R^{(a)}=R_{\alpha_{a}^{1}}=R_{\alpha_{a}^{2}} \cdots=R_{\alpha_{a}^{l_{a}}}
$$

We can re-write the counting as

Number of color symmetrised tensor invariants of rank $d=$

$$
\frac{1}{(n !)^{2}} \sum_{p \vdash d} \sum_{\substack{R_{i, j} \vdash d \\\left\{1 \leq i \leq d, 1 \leq j \leq p_{i}\right\}}} \sum_{\sigma_{1} \in S_{n}} \sum_{\sigma_{2} \in S_{n}} \frac{1}{|\operatorname{Sym}(p)|} \prod_{i=1}^{d} \prod_{j=1}^{p_{i}} \chi^{R_{i, j}}\left(\sigma_{1}^{i}\right) \chi^{R_{i, j}}\left(\sigma_{2}^{i}\right)
$$

We have collected from (7.84) all the terms coming from a fixed conjugacy class of $\alpha$, which corresponds to a partition $p$ of $d$, specified by multiplicities $p_{i}$ of cycles lengths $i$ in the permutation $\alpha$, i.e. $\sum i p_{i}=d$. Given the delta functions on the representations, for a given $p$, the number of distinct representations being summed after using these delta functions 
is $\sum_{i=1}^{d} p_{i}$. We denote these representation labels $R_{i, j}$, where $i$ runs over the possible cycle lengths and $j$ runs over the distinct cycles of the same length $i$.

This is also the dimension of the projection of $\mathcal{K}(n, d)$ to the subspace belonging to the one-dimensional irrep of $S_{d}$. We have in general

$$
\mathcal{K}(n, d)=\bigoplus_{Y \vdash d}(\mathcal{K}(n, d))_{Y}
$$

The above gives the projection to the $S_{d}$ invariant subspace.

$\left.\operatorname{dim}(\mathcal{K}(n, d))_{Y=[n]}\right)=$ Number of color symmetrised tensor invariants of rank $d$

$$
\frac{1}{(n !)^{2}} \sum_{p \vdash d} \sum_{\substack{R_{i, j} \vdash d \\\left\{1 \leq i \leq d, 1 \leq j \leq p_{i}\right\}}} \sum_{\sigma_{1} \in S_{n}} \sum_{\sigma_{2} \in S_{n}} \frac{1}{|\operatorname{Sym}(p)|} \prod_{i=1}^{d} \prod_{j=1}^{p_{i}} \chi^{R_{i, j}}\left(\sigma_{1}^{i}\right) \chi^{R_{i, j}}\left(\sigma_{2}^{i}\right)
$$

For general representations $Y$, we have

$$
\begin{aligned}
& \operatorname{dim}\left((\mathcal{K}(n, d))_{Y}\right) \\
& =\frac{d(Y)}{(n !)^{2}} \sum_{p \vdash d} \chi^{Y}\left(\sigma_{p}\right) \sum_{\substack{R_{i, j} \vdash d \\
\left\{1 \leq i \leq d, 1 \leq j \leq p_{i}\right\}}} \sum_{\sigma_{1} \in S_{n}} \sum_{\sigma_{2} \in S_{n}} \frac{1}{|\operatorname{Sym}(p)|} \prod_{i=1}^{d} \prod_{j=1}^{p_{i}} \chi^{R_{i, j}}\left(\sigma_{1}^{i}\right) \chi^{R_{i, j}}\left(\sigma_{2}^{i}\right)
\end{aligned}
$$

For practical computations, expressions for the $S_{p}^{(d)}(n)$ were also given in [41], for $d \leq 4$, in terms of sums over partitions of $n$, with weights obtained by applying appropriate substitutions to the generating function of cycle indices of $S_{n}$ (equations (63) and (77) of arXiv version). The generalization of these expressions in terms of partition sums to general $d$ is left as an interesting exercise for the future.

\section{7 $\mathcal{K}^{\infty}$ and color symmetry}

We explained in section 5.4 that the infinite direct sum $\mathcal{K}^{\infty}$

$$
\mathcal{K}^{\infty}=\bigoplus_{n=0}^{\infty} \mathcal{K}(n)
$$

has two products, which both play a role in correlators. If we restrict to the colorsymmetrised subspace

$$
\mathcal{K}_{Y_{0}}^{\infty}=\bigoplus_{n=0}^{\infty} \mathcal{K}_{Y_{0}}(n)
$$

we again have a vector space with two products. We have already seen that the product at fixed $n$ of two elements in $\mathcal{K}_{Y_{0}}(n)$ is in $\mathcal{K}_{Y_{0}}(n)$. Likewise the outer product of two color-symmetrized elements in $\mathcal{K}_{Y_{0}}\left(n_{1}\right)$ and $\mathcal{K}_{Y_{0}}\left(n_{2}\right)$ is a color-symmetrized element in $\mathcal{K}_{Y_{0}}\left(n_{1}+n_{2}\right)$. An easy way to see this is to think about the multiplication of colorsymmetrized observables. Systematic investigations of color-symmetrized correlators is left for the future. We expect that the $\mathcal{K}^{\infty}$ will prove to be a useful tool in these investigations. 


\section{Summary and discussion}

\subsection{Summary}

We have developed the description of the counting and correlators of general gauge invariant observables in a class of tensor models started in [41]. We focus on bosonic tensor models with a complex scalar field having $d$ indices. We have showed that the permutation centralizer algebras introduced in [52] provide a powerful framework for elucidating many aspects of correlators in the Gaussian model. The vector space of gauge-invariant observables in the rank-3 tensor model is isomorphic to the vector space of the algebra $\mathcal{K}(n)$. This algebra is spanned by elements in $\mathbb{C}\left(S_{n}\right) \otimes \mathbb{C}\left(S_{n}\right)$ which commute with the diagonally embedded $\mathbb{C}\left(S_{n}\right)$. There is a also an equivalent description in terms of the subspace of $\mathbb{C}\left(S_{n}\right) \otimes \mathbb{C}\left(S_{n}\right) \otimes \mathbb{C}\left(S_{n}\right)$ which is invariant under left and right action of the diagonal $\mathbb{C}\left(S_{n}\right)$.

$\mathcal{K}(n)$ is a semi-simple associative algebra, i.e. an associative algebra with nondegenerate bilinear pairing. As a result, by the Wedderburn-Artin theorem, it is isomorphic to a direct sum of matrix algebras. The number of blocks in $\mathcal{K}(n)$ is the number of ordered triples $\left[R_{1}, R_{2}, R_{3}\right]$ of Young diagrams with $n$ boxes which have a non-vanishing Kronecker coefficient. The sizes of the blocks are the Kronecker coefficients $\mathrm{C}\left(R_{1}, R_{2}, R_{3}\right)$. The basis elements corresponding to the matrix decomposition are constructed using Clebsch-Gordan coefficients for the invariant in $R_{1} \otimes R_{2} \otimes R_{3}$. These basis elements of $\mathcal{K}(n)$ correspond to gauge invariant observables which diagonalize the 2-point function of normal ordered observables. A subspace of observables corresponds to the centre of $\mathcal{K}(n)$. These observables can be constructed without the detailed knowledge of Clebsch-Gordan coefficients. They only require characters of $S_{n}$. A basis for the centre is given by triples of Young diagrams $R_{1}, R_{2}, R_{3}$ which have non-vanishing $\mathrm{C}\left(R_{1}, R_{2}, R_{3}\right)$. One point functions of central observables are proportional to $\mathrm{C}\left(R_{1}, R_{2}, R_{3}\right)$.

The above results are based on a few key ingredients: the parameterization of gauge invariant observables using equivalence classes of permutations, the use of representation theory to give a Fourier transformed description of observables in terms of Young diagrams, and Clebsch-Gordan coefficients. These methods have found extensive use in multi-matrix models over the recent years (an overview is in [51]).

The algebra $\mathcal{K}(n)$ allows a systematic study of the implications of the color-exchange symmetry in tensor models. In [41] we had described counting formulae for colorsymmetrized observables, which correspond to color-symmetrized graphs. Here we give the complete decomposition of $\mathcal{K}(n)$ in terms of a direct sum, labelled by Young diagrams of the $S_{3}$ color-exchange symmetry.

$$
\mathcal{K}(n)=\bigoplus_{Y \vdash 3} \mathcal{K}_{Y}(n)
$$

The color-symmetrized subspace $\mathcal{K}_{Y_{0}}(n)$ is a closed sub-algebra of $\mathcal{K}(n)$. Again as a result of the WA theorem, we immediately expect that it should be a direct sum of matrix algebras. The corresponding counting formula as a sum of squares is given in (7.61), and the corresponding refined Clebsch-Gordan coefficients are described. Similar group theoretic decompositions for $\mathcal{K}_{Y}(n)$ are given in section 7 . 
The counting formulae for $\mathcal{K}_{Y_{0}}(n)$ can be expressed in terms of sums over characters of $S_{n}$ parametrized by partitions of $S_{d}$ (with $d=3$ ). Such formulae were derived using the Burnside lemma in [41] for $d=3,4$ and used to get explicit number sequences for dimensions of the space of color-symmetrized observables (color-symmetrized graphs). We have generalized these character formulae (section 7.6) to general $d$, by exploiting an alternative derivation which makes use the representation theoretic Fourier basis for $\left(\mathbb{C}\left(S_{n}\right)\right)^{\otimes d}$. The group theoretic results we have developed for color-exchange symmetry will be useful for the study of correlators in Gaussian models, as well as interacting models which are perturbations of Gaussians by color-symmetrized observables. This is an interesting direction for future investigations.

A number of other future research directions are suggested by the results of this paper. We outline some of them below.

\subsection{Towards Young diagram statistical models and field theory}

We have found above that interesting classes of observables in tensor models, related to the centres of permutation algebras built from equivalence classes of permutations describing general observables, are parametrized by sets of Young diagrams. Their correlators are directly related to fundamental representation theoretic quantities, e.g. (5.9). Similar observations in the context of multi-matrix models are developed in [52]. This leads us to a natural question: is there a statistical model of Young diagrams (YD) for which the functions (5.9) are the correlators? Section 6 describes a mapping of these correlators observables in a topological field theory on 2-complexes. Here we explore a different perspective, and provide a partial answer of the above question of what this statistical/field theory model could be.

Fix $n \in \mathbb{N}$, and consider $R \vdash n$ a Young diagram. A real field over YDs is a function $Y: p(n) \rightarrow \mathbb{R}$. We define an action of a $Y^{k}-$ Young diagram model (YDM) by:

$$
S_{k-\mathrm{YDM}}[Y]=\sum_{R, R^{\prime} \vdash n} Y_{R} K\left(R, R^{\prime}\right) Y_{R^{\prime}}+g \sum_{R_{l} \vdash n} I\left(\left\{R_{l}\right\}\right) \prod_{l=1}^{k} Y_{R_{l}}
$$

where $K\left(R, R^{\prime}\right)$ and $I\left(\left\{R_{l}\right\}\right)$ are kernels, $g$ a coupling constant. From now, let us restrict to a cubic action determined by $k=3, K\left(R, R^{\prime}\right)=\delta_{R, R^{\prime}}$ and $I\left(\left\{R_{l}\right\}\right)=\mathrm{C}\left(R_{1}, R_{2}, R_{3}\right)$, the Kronecker coefficient. The use of complex fields, the choice of $I\left(\left\{R_{l}\right\}\right)$ as the LittlewoodRichardson coefficient, or more generic $Y^{k}$-models of the form $I\left(\left\{R_{l}\right\}\right)=\mathrm{C}_{k}\left(R_{1}, R_{2}, \ldots, R_{k}\right)$, see (5.9), might be also interesting choices let for future investigations.

Being interested in perturbation theory, the positivity of the above action will not be addressed here. The partition function of the model (8.2) under the above restrictions is of the form

$$
Z_{3-\mathrm{YDM}}[g, J]=\int \prod_{R \vdash n} d Y_{R} e^{-S_{3-\mathrm{YDM}}[Y]-\sum_{R \vdash n} J_{R} Y_{R}}
$$

where $J_{R}$ is a source term. Correlators are computed perturbatively using the Gaussian 
measure $d \mu(Y)=\prod_{R \vdash n} d Y_{R} e^{-\sum_{R}\left|Y_{R}\right|^{2}}$ and we find

$$
\begin{aligned}
& \left\langle Y_{S_{1}} Y_{S_{2}} \ldots Y_{S_{k}}\right\rangle_{3-\mathrm{YDM}} \\
& \quad=\sum_{n=0}^{\infty} \frac{(-g)^{n}}{n !} \int d \mu(Y)\left(Y_{S_{1}} Y_{S_{2}} \ldots Y_{S_{k}}\right)\left(\sum_{R_{l} \vdash n} \mathrm{C}\left(R_{1}, R_{2}, R_{3}\right) Y_{R_{1}} Y_{R_{2}} Y_{R_{3}}\right)^{n} .
\end{aligned}
$$

The free propagator in this theory is naturally defined by $\left\langle Y_{R} Y_{R^{\prime}}\right\rangle_{\text {free }}=G_{0}\left(R, R^{\prime}\right)=\delta_{R R^{\prime}}$. Via the Wick theorem, $N$-point correlators expand in terms of Feynman graphs, as $\left\langle Y_{S_{1}} Y_{S_{2}} \ldots Y_{S_{N}}\right\rangle_{3 \text {-YDM; pert. }}=\sum_{\mathcal{G}} K_{\mathcal{G}} A_{\mathcal{G}}$, with $A_{\mathcal{G}}\left(S_{1} . S_{2}, \ldots, S_{N}\right)$ a graph amplitude, and $K_{\mathcal{G}}$ a combinatorial factor.

We evaluate a connected 3-point function a first order of perturbation:

$$
\begin{aligned}
& \left\langle Y_{S_{1}} Y_{S_{2}} Y_{S_{3}}\right\rangle_{3 \text {-YDM; pert.; connected }}=-g \sum_{R_{l}} \mathrm{C}\left(R_{1}, R_{2}, R_{3}\right) \prod_{\sigma \in S_{3}} \prod_{i} G_{0}\left(S_{i}, R_{\sigma(i)}\right) \\
& =-3 ! g \mathrm{C}\left(R_{1}, R_{2}, R_{3}\right) \text {. }
\end{aligned}
$$

There are 3 ! trees contributing to the correlator and each of them has the same weight $-g \mathrm{C}\left(R_{1}, R_{2}, R_{3}\right)$. Thus at this first order of perturbation $\left\langle Y_{S_{1}} Y_{S_{2}} Y_{S_{3}}\right\rangle_{3 \text {-YDM; pert.; connected }}$ is proportional to $\left\langle\mathcal{O}_{S_{1}, S_{2}, S_{3}}\right\rangle$ up to the factor $\frac{1}{(-3 ! g)(n !)^{2}}\left[\prod_{i=1}^{3} f_{N}\left(S_{l}\right)\right]$.

Computing a connected 4-point function $\left\langle Y_{S_{1}} Y_{S_{2}} Y_{S_{3}} Y_{S_{4}}\right\rangle_{3 \text {-YDM; pert.; connected at second }}$ order of perturbation, we have a sum of Feynman amplitudes. Consider the tree graph

which appears in that expansion,

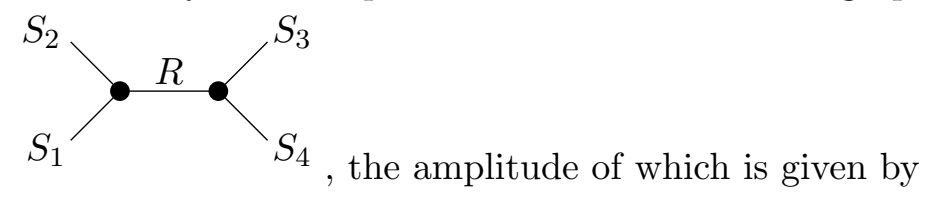

$$
A_{t}\left(S_{1} . S_{2}, S_{3}, S_{4}\right)=\frac{g^{2}}{2} \sum_{R \vdash n} \mathrm{C}\left(S_{1}, S_{2}, R\right) \mathrm{C}\left(R, S_{3}, S_{4}\right)
$$

which is proportional to $\mathcal{O}_{S_{1}, S_{2}, S_{3}, S_{4}}$ (5.9). We conjecture that at any order $m-2>0$ of perturbation theory, $\left\langle\mathcal{O}_{S_{1}, S_{2}, \ldots, S_{m}}\right\rangle$ corresponds to a tree (hence connected) Feynman graph of the correlator $\left\langle Y_{S_{1}} Y_{S_{2}} \ldots Y_{S_{m}}\right\rangle_{3-\mathrm{YDM}}$; pert.;connected up to a constant.

Dealing with an action $S_{d \text {-YDM }}$, it is direct to get at first order of perturbation:

$$
\left\langle Y_{S_{1}} Y_{S_{2}} \ldots Y_{S_{d}}\right\rangle_{d-\text { YDM } \text {; pert.; connected }} \propto\left\langle\mathcal{O}_{S_{1}, S_{2}, \ldots, S_{d}}\right\rangle
$$

Beyond tree level, generic amplitudes should involve free sums over Young diagrams associated with loops in the graphs, hence factors of the number of partitions $p(n)$. In the limit $n \rightarrow \infty$, where we have an infinite number of degrees of freedom, one should expect that a YDM will have divergent amplitudes. It will be interesting to investigate the application of quantum field theoretic renormalization techniques to make sense of this limit.

\subsection{The space of holographic duals of tensor models}

In the early applications of matrix models as holographic duals to quantum gravity in low dimensions of the nineties [1,2], a detailed map was achieved where the holographic duals 
included minimal model CFTs with $c<1$ coupled to Liouville theory and the standard string theory $b, c$ ghost system, as well as a $c=-2$ model coupled to Liouville and ghosts as a dual for the Gaussian model [79]. A dual to the Gaussian model in terms of Belyi maps and topological strings on $\mathbb{C P}^{1}$ has also been investigated [67, 80-82], which should be related to the earlier $c=-2$ proposal.

The AdS2 dual for the double-scaled limit with a quartic interaction (Gurau-Witten model) is currently of active interest, with motivations from black hole physics [28, 29]. The rich mathematical structure of the Gaussian model raises the very interesting question of what is the precise dual of this model. The description of permutation TFT2 constructions for the correlators of the Gaussian model given in section 6 is a good starting point for investigations along the lines of $[67,80-82]$. The rich mathematics involving permutations, Fourier transforms of group algebras, the structure of associative algebras, the role of color-exchange symmetry underlying the space of tensor model observables suggests that a complete description of holography for the space of tensor models will be a fascinating challenge.

\subsection{Computational complexity of central correlators in matrix versus tensor models}

One of the interesting results is that the one-point function in the representation basis, for the $d=3$ complex tensor model, is equal to the Kronecker coefficient (5.5), a number of fundamental importance in Computational Complexity Theory.

Compare this with the extremal 3-point correlator for the half-BPS sector which is directly proportional to the Littlewood-Richardson (LR) coefficient $g(R, S, T)$ [42]

$$
\left\langle\chi^{R}(Z) \chi^{S}(Z) \chi^{T}\left(Z^{\dagger}\right)\right\rangle=g(R, S, T) \frac{n ! \operatorname{Dim}_{N}(T)}{d(T)}
$$

This correlator has been interpreted in terms of topology change [83]. The half-BPS sector and its connections to topology change has also been investigated recently in [84, 85]. In the AdS-CFT correspondence, Young diagrams can be used to parametrize space-times of different topologies $[42,86,87]$.

The LR coefficient has been of interest in the context of Computational Complexity Theory. It has been shown that the determination of the vanishing or otherwise of the LR coefficient can be done in polynonial time [88]. The actual evaluation of the LR coefficient for general Young diagrams is \# P-hard [89] (\# P is the analog of NP when we go from decision problems to counting problems). Recently it was found that deciding the vanishing of Kronecker coefficients is NP-hard [90]. This is an interesting contrast between central correlators in the 1-matrix problem (8.8) and the one-point function of central observables in the tensor model (5.5). Characterizing the complexity of determining the vanishing of extremal correlators of central observables in the 2-matrix case studied in [52] would be a useful problem to solve in getting a more complete picture of the relative complexities in matrix and tensor models.

It would be interesting to explore the implications of the above results in the context of physical applications of matrix/tensor models, e.g. in black hole physics or early cos- 
mology. Different physical roles for computational complexity in these contexts have been proposed [91, 92]. Finite $N$ effects, of interest in the physics of the stringy exclusion priinciple and giant gravitons, turn out to have drastic effects on the complexity questions [93], tending to allow polynomial time algorithms.

\section{Acknowledgments}

SR's research is supported by the STFC consolidated grant ST/L000415/1 "String Theory, Gauge Theory \& Duality" at Queen Mary University of London and a Visiting Professorship at the Mandelstam Institute for Theoretical Physics, University of the Witwatersrand, funded by a Simons Foundation grant. JBG thanks the Centre for Research in String Theory at QMUL for hospitality, while this work was in progress.

\section{A Symmetric group, representation theory and group algebra}

\section{A.1 Symmetric group and representation}

We collect some basic facts in the representation theory of symmetric groups. A useful textbook discussion is in [77].

Irreducible representations of symmetric group $S_{n}$ are labelled by Young diagrams or partitions $R$ of $n$, that we denote $R \vdash n$. In the following, we interchangeably use and assimilate an irrep with $R$. As a consequence of the Schur-Weyl duality, one associates also a Young diagram $R$ with an irreducible representation of the unitary group $\mathrm{U}(N)$, when the length $l(R)$ of the first column of $R$ is bounded by $N$, i.e $l(R) \leq N$.

At fixed $n$, denote $d(R)$ the dimension of the representation of $S_{n}$ and $\operatorname{Dim}_{N}(R)$ the dimension of representation of $\mathrm{U}(N)$, we write

$$
d(R)=n ! / h(R), \quad \operatorname{Dim}_{N}(R)=f_{N}(R) / h(R)
$$

where $h(R)$ is the product of the so-called hook lengths, i.e. $h(R)=\prod_{i, j}\left(c_{j}-j+r_{i}-i+1\right)$ and $f_{N}(R)$ is the products of box weights

$$
f_{N}(R)=\prod_{i, j}(N-i+j)
$$

where the pairs $(i, j)$ label the boxes of the Young diagram: $i$ is the row label and $j$ is the column label. $r_{i}$ is the row length of the $i$ 'th row. $c_{j}$ is the column length of the $j$ 'th column.

The matrices $D_{i j}^{R}(\sigma)$ of the representation $R$ of a permutation $\sigma \in S_{n}$ are $d(R) \times d(R)$ and satisfy the following basic properties

$$
\sum_{i} D_{a i}^{R}(\sigma) D_{i b}^{R}\left(\sigma^{\prime}\right)=D_{a b}^{R}\left(\sigma \sigma^{\prime}\right), \quad D_{a b}^{R}(e)=\delta_{a b}
$$

and are also orthogonal

$$
\sum_{\sigma \in S_{n}} D_{i j}^{R}(\sigma) D_{k l}^{S}(\sigma)=\frac{n !}{d(R)} \delta_{R S} \delta_{i k} \delta_{j l}
$$


This follows from Schur's lemma. Note that we choose to work with orthogonal (and so real) matrices obeying

$$
D_{i j}^{R}\left(\sigma^{-1}\right)=D_{j i}^{R}(\sigma)
$$

such that (A.4) is again $\sum_{\sigma} D_{i j}^{R}(\sigma) D_{l k}^{S}\left(\sigma^{-1}\right)$.

Another important object in representation theory is of course the character of a given representation. The character of the irrep $R$ is simply the trace of $D^{R}(\sigma), \chi^{R}(\sigma)=$ $\operatorname{Tr}\left(D^{R}(\sigma)\right)=\sum_{i} D_{i i}^{R}(\sigma)$. It is immediate that

$$
\chi^{R}(\sigma)=\chi^{R}\left(\sigma^{-1}\right)
$$

The Kronecker delta of the symmetric group (defined to be equal to 1 when the argument is the identity and 0 otherwise) decomposes as

$$
\delta(\sigma)=\sum_{R \vdash n} \frac{d(R)}{n !} \chi^{R}(\sigma)
$$

The summation $R \vdash n$ is a sum over partitions of $R$ of $n$, equivalently over Young diagrams with $n$ boxes. We have also

$$
\begin{aligned}
\sum_{\gamma \in S_{n}} \delta\left(\gamma \sigma \gamma^{-1} \tau^{-1}\right) & =\sum_{\gamma \in S_{n}} \sum_{R \vdash n} \frac{d(R)}{n !} \sum_{i, a, b, c} D_{i a}^{R}(\gamma) D_{a b}^{R}(\sigma) D_{c b}^{R}(\gamma) D_{i c}^{R}(\tau) \\
\stackrel{(\mathrm{A} .4)}{=} & \sum_{R \vdash n} \sum_{i, a, b, c} D_{a b}^{R}(\sigma) D_{i c}^{R}(\tau) \delta_{i c} \delta_{a b}=\sum_{R \vdash n} \chi^{R}(\sigma) \chi^{R}(\tau)
\end{aligned}
$$

If $B$ is a central element, then

$$
\begin{aligned}
\sum_{\gamma \in S_{n}} \chi^{R}\left(A \gamma B \gamma^{-1}\right) & =n ! \chi^{R}(A B)=\sum_{a, b, c, d} \sum_{\gamma \in S_{n}} D_{a b}^{R}(A) D_{b c}^{R}(\gamma) D_{c d}^{R}(B) D_{d a}^{R}\left(\gamma^{-1}\right) \\
& \stackrel{(\mathrm{A} .4)}{=} \frac{n !}{d(R)} \sum_{a, b, c, d} D_{a b}^{R}(A) D_{c d}^{R}(B) \delta_{b a} \delta_{c d}=\frac{n !}{d(R)} \chi^{R}(A) \chi^{R}(B)
\end{aligned}
$$

Hence

$$
\chi^{R}(A B)=\frac{1}{d(R)} \chi^{R}(A) \chi^{R}(B)
$$

Also useful to know that

$$
\frac{1}{n !} \sum_{\alpha} \chi^{R}(\alpha) N^{\mathbf{c}(\alpha)}=\operatorname{Dim}_{N}(R)
$$

where $\operatorname{Dim}_{N}(R)$ is the dimension of the $\mathrm{U}(N)$ representation $R$ (A.1).

The same type of the above calculation which involves (A.4) leads to other formulae given by

$$
\sum_{\sigma \in S_{n}} \chi^{R}\left(\sigma \tau_{1}\right) \chi^{S}\left(\sigma \tau_{2}\right)=\frac{n !}{d(R)} \delta_{R S} \chi^{R}\left(\tau_{1} \tau_{2}^{-1}\right) \stackrel{\tau_{1}=\tau_{2}=\mathrm{id}}{\Rightarrow} \sum_{\sigma \in S_{n}} \chi^{R}(\sigma) \chi^{S}(\sigma)=n ! \delta_{R S}
$$


Finally, concerning identities involving the dimension of irrep $R$ in $\mathrm{U}(N)$, one has

$$
\sum_{\sigma \in S_{n}} D_{i j}^{R}(\sigma) N^{\mathbf{c}(\sigma)}=\delta_{i j} f_{N}(R) \stackrel{(\mathrm{A} .4)}{\Rightarrow} \sum_{\sigma \in S_{n}} \chi^{R}(\sigma) N^{\mathbf{c}(\sigma)}=d(R) f(R)=n ! \operatorname{Dim}_{N}(R)
$$

where $\mathbf{c}(\sigma)$ is the number of cycles of $\sigma$.

The following table lists the above formulas:

$$
\begin{aligned}
D_{i j}^{R}\left(\sigma^{-1}\right) & =D_{j i}^{R}(\sigma) \\
\chi^{R}(\sigma) & =\chi^{R}\left(\sigma^{-1}\right)=\chi^{R}\left(\gamma \sigma \gamma^{-1}\right), \quad \forall \gamma \in S_{n} \\
\delta(\sigma) & =\sum_{R \vdash n} \frac{d(R)}{n !} \chi^{R}(\sigma) \\
\sum_{\sigma \in S_{n}} D_{i j}^{R}(\sigma) D_{k l}^{S}(\sigma) & =\frac{n !}{d(R)} \delta_{R S} \delta_{i k} \delta_{j l} \\
\sum_{\sigma \in S_{n}} \chi^{R}\left(\sigma \tau_{1}\right) \chi^{S}\left(\sigma \tau_{2}\right) & =\frac{n !}{d(R)} \delta_{R S} \chi\left(\tau_{1} \tau_{2}^{-1}\right) \\
\sum_{\sigma \in S_{n}} \chi^{R}(\sigma) \chi^{S}(\sigma) & =n ! \delta_{R S} \\
\forall B & \in \mathcal{Z}\left(S_{n}\right), \quad \chi^{R}(A B)=\frac{1}{d(R)} \chi^{R}(A) \chi^{R}(B) \\
\sum_{\gamma \in S_{n}} \delta\left(\gamma \sigma \gamma^{-1} \tau^{-1}\right) & =\sum_{R \vdash n} \chi^{R}(\sigma) \chi^{R}(\tau) \\
\sum_{\sigma \in S_{n}} D_{i j}^{R}(\sigma) N^{\mathbf{c}(\sigma)} & =\delta_{i j} f_{N}(R) \\
\sum_{\sigma \in S_{n}} \chi^{R}(\sigma) N^{\mathbf{c}(\sigma)} & =d(R) f(R)=n ! \operatorname{Dim}_{N}(R)
\end{aligned}
$$

Defining the central element $\Omega \in \mathbb{C}\left(S_{n}\right)$,

$$
\Omega=\sum_{\sigma \in S_{n}} N^{n-\mathbf{c}(\sigma)} \sigma
$$

equation (A.23), can be also written as

$$
\frac{N^{n}}{n !} \chi^{R}(\Omega)=\operatorname{Dim}_{N}(R)
$$

\section{A.2 Clebsch-Gordan coefficients}

Consider two irreps $V_{R_{1}}, V_{R_{2}}$ of $S_{n}$ corresponding to Young diagrams $R_{1}, R_{2}$. We assume that we have picked an orthogonal basis of states for the irreps e.g. $|R, i\rangle$ obeying

$$
\langle R, j \mid R, i\rangle=\delta_{i j}
$$

A representation $\varrho_{R}: S_{n} \rightarrow \operatorname{End}\left(V_{R}\right)$ is given by a matrix $D^{R}$ with entries determined by $\varrho_{S}(\sigma)|R, i\rangle=\sum_{l=1}^{d(R)} D_{l i}^{R}(\sigma)|R, l\rangle$ with $\sigma \in S_{n}$. We write in short $\varrho_{S}(\sigma)=\sigma$ and then 
$\langle R, j|\sigma| R, i\rangle=D_{j i}^{R}(\sigma)$. The tensor product representation $V_{R_{1}} \otimes V_{R_{2}}$ can be decomposed into a direct sum of irreps $V_{R_{3}}$ with multiplicities

$$
V_{R_{1}} \otimes V_{R_{2}}=\bigoplus_{R_{3} \vdash n} V_{R_{3}} \otimes V_{R_{3}}^{\mathrm{m}}
$$

One set of basis vectors in the tensor product space is $\left|R_{1}, i_{1}\right\rangle \otimes\left|R_{2}, i_{2}\right\rangle=:\left|R_{1}, i_{1} ; R_{2}, i_{2}\right\rangle$ while the r.h.s. corresponds to a basis set $\left|R_{3}, i_{3}, \tau_{R_{3}}\right\rangle$. The label $i_{3}$ runs over states in the irrep $R_{3}$, while $\tau_{R_{3}}$ runs over an orthogonal basis in the multiplicity space $V_{R_{3}}^{\mathrm{m}}$. ClebschGordan coefficients (CG's) are transition coefficients between the two types of bases

$$
C_{i_{1}, i_{2} ; i_{3}}^{R_{1}, R_{2} ; R_{3}, \tau_{R_{3}}}:=\left\langle R_{1}, i_{1} ; R_{2}, i_{2} \mid R_{3}, \tau_{R_{3}}, i_{3}\right\rangle=\left\langle R_{3}, \tau_{R_{3}}, i_{3} \mid R_{1}, i_{1} ; R_{2}, i_{2}\right\rangle
$$

The last relation is obtained from the reality property of the CG's. We will then use $C_{i_{1}, i_{2} ; i_{3}}^{R_{1}, R_{2} ; R_{3}, \tau_{R_{3}}}=C_{i_{3} ; i_{1}, i_{2}}^{\tau_{R_{3}}, R_{3} ; R_{1}, R_{2}}$. A detailed discussion of the CG's for symmetric groups is in [77].

Linear operators for $\sigma \otimes \sigma$ in $V_{S} \otimes V_{R}$ have matrix elements

$$
D_{i_{1} j_{1}}^{R_{1}}(\sigma) D_{i_{2} j_{2}}^{R_{2}}(\sigma)=\left\langle R_{1}, i_{1}|\sigma| R_{1}, j_{1}\right\rangle\left\langle R_{2}, i_{2}|\sigma| R_{2}, j_{2}\right\rangle=:\left\langle R_{1}, i_{1} ; R_{2}, i_{2}|\sigma| R_{1}, j_{1} ; R_{2}, j_{2}\right\rangle
$$

Inserting a complete set of states resolving the identity we get

$$
\begin{aligned}
& D_{i_{1} j_{1}}^{R_{1}}(\sigma) D_{i_{2} j_{2}}^{R_{2}}(\sigma)=\sum_{R_{3}, R_{3}^{\prime}, \tau_{R_{3}}, \tau_{R_{3}}^{\prime}} \sum_{i_{3}, j_{3}}\left\langle R_{1}, i_{1} ; R_{2}, i_{2} \mid R_{3}, \tau_{R_{3}}^{\prime}, i_{3}\right\rangle\left\langle R_{3}, \tau_{R_{3}}, i_{3}|\sigma| R_{3}^{\prime}, \tau_{R_{3}^{\prime}}^{\prime}, j_{3}\right\rangle \\
& \times\left\langle R_{3}^{\prime}, \tau_{R_{3}^{\prime}}^{\prime}, j_{3} \mid R_{1}, j_{1} ; R_{2}, j_{2}\right\rangle \\
& =\sum_{R_{3}, R_{3}^{\prime}, \tau_{R_{3}}, \tau_{R_{3}}^{\prime}} \sum_{i_{3}, j_{3}}\left\langle R_{1}, i_{1} ; R_{2}, i_{2} \mid R_{3}, \tau_{R_{3}}, i_{3}\right\rangle \delta_{R_{3} R_{3}^{\prime}} \delta_{\tau_{R_{3}} \tau_{R_{3}^{\prime}}^{\prime}} D_{i_{3} j_{3}}^{R_{3}}(\sigma)\left\langle R_{3}^{\prime}, \tau_{R_{3}^{\prime}}^{\prime}, j_{3} \mid R_{1}, i_{2} ; R_{2}, j_{2}\right\rangle \\
& =\sum_{R_{3}, \tau_{R_{3}}} \sum_{i_{3}, j_{3}}\left\langle R_{1}, i_{1} ; R_{2}, i_{2} \mid R_{3}, \tau_{R_{3}}, i_{3}\right\rangle D_{i_{3} j_{3}}^{R_{3}}(\sigma)\left\langle R_{3}, \tau_{R_{3}}, j_{3} \mid R_{1}, j_{1} ; R_{2}, j_{2}\right\rangle
\end{aligned}
$$

Using the definition of the CG's (A.30), can be also written as

$$
D_{i_{1} j_{1}}^{R_{1}}(\sigma) D_{i_{2} j_{2}}^{R_{2}}(\sigma)=\sum_{R_{3}, \tau} \sum_{i_{3}, j_{3}} C_{i_{1}, i_{2} ; i_{3}}^{R_{1}, R_{2} ; R_{3}, \tau_{R_{3}}} D_{i_{3} j_{3}}^{R_{3}}(\sigma) C_{j_{1}, j_{2} ; j_{3}}^{R_{1}, R_{2} ; R_{3}, \tau_{R_{3}}}
$$

Because there is no possible confusion, $\tau_{R_{3}}$ is sometimes denoted $\tau$ in the text.

The following identities hold

$$
\begin{aligned}
\sum_{j_{1}, j_{2}} D_{i_{1} j_{1}}^{R_{1}}(\gamma) D_{i_{2} j_{2}}^{R_{2}}(\gamma) C_{j_{1}, j_{2} ; j_{3}}^{R_{1}, R_{2} ; R_{3}, \tau} & =\sum_{i_{3}} C_{i_{1}, i_{2} ; i_{3}}^{R_{1}, R_{2}, R_{3}, \tau} D_{i_{3} j_{3}}^{R}(\gamma) \\
\sum_{i_{1}, i_{2}} C_{i_{1}, i_{2} ; i_{3}}^{R_{1}, R_{2} ; R_{3}, \tau} C_{i_{1}, i_{2} ; j_{3}}^{R_{1}, R_{2} ; R_{3}^{\prime}, \tau^{\prime}} & =\delta_{R_{3} R_{3}^{\prime}} \delta_{\tau \tau^{\prime}} \delta_{i_{3} j_{3}} \\
\sum_{R_{3}, \tau ; i_{3}, j_{3}} C_{i_{1}, i_{2} ; i_{3}}^{R_{1}, R_{2} ; R_{3}, \tau} C_{j_{1}, j_{2} ; i_{3}}^{R_{1}, R_{2} ; R_{3}, \tau} & =\delta_{i_{1} j_{1}} \delta_{i_{2} j_{2}} \\
\sum_{i_{1}, i_{2} ; i_{3}}^{R_{1}, R_{2} ; R_{3}, \tau} D_{i_{3} j_{3}}^{R_{3}}(\gamma) C_{j_{1}, j_{2} ; j_{3}}^{R_{1}, R_{2} ; R_{3}, \tau} & =D_{i_{1} j_{1}}^{R_{1}}(\gamma) D_{i_{2} j_{2}}^{R_{2}}(\gamma)
\end{aligned}
$$

Note that (A.31) is (A.35). 
Furthermore, we have by applying twice (A.32):

$$
\begin{aligned}
& \sum_{j_{1}, j_{2}, j_{3}} D_{i_{1} j_{1}}^{R_{1}}(\gamma) D_{i_{2} j_{2}}^{R_{2}}(\gamma) D_{i_{3} j_{3}}^{R_{3}}(\gamma) C_{j_{1}, j_{2} ; j_{3}}^{R_{1}, R_{2} ; R_{3}, \tau}=\sum_{j_{3}} \sum_{l} C_{i_{1}, i_{2} ; l}^{R_{1}, R_{2} ; R_{3}, \tau} D_{l j_{3}}^{R_{3}}(\gamma) D_{i_{3} j_{3}}^{R_{3}}(\gamma) \\
& =\sum_{l} C_{i_{1}, i_{2} ; l}^{R_{1}, R_{2} ; R_{3}, \tau} \sum_{j_{3}} D_{l j_{3}}^{R_{3}}(\gamma) D_{j_{3} i_{3}}^{R_{3}}\left(\gamma^{-1}\right)=C_{i_{1}, i_{2} ; i_{3}}^{R_{1}, R_{2} ; R_{3}, \tau}
\end{aligned}
$$

These equations can be put in diagrammatrics which lighten the proofs. We now recall them. The diagrammatic notation for the CG coefficient will be a three valent black node:

$$
C_{i_{1}, i_{2} ; i_{3}}^{R_{2}, R_{2} ; R_{3}, \tau}=\overbrace{i_{2}}^{i_{R_{2}}^{i_{1}} \overbrace{\tau}^{R_{1}} R_{3}} i_{3}
$$

A representation matrix $D_{i j}^{R}(\sigma)$ is drawn like $-\sigma-$, the rest of the indices will be explicit when the matrix will be composed with others coefficients. Then the above identities can be translated as
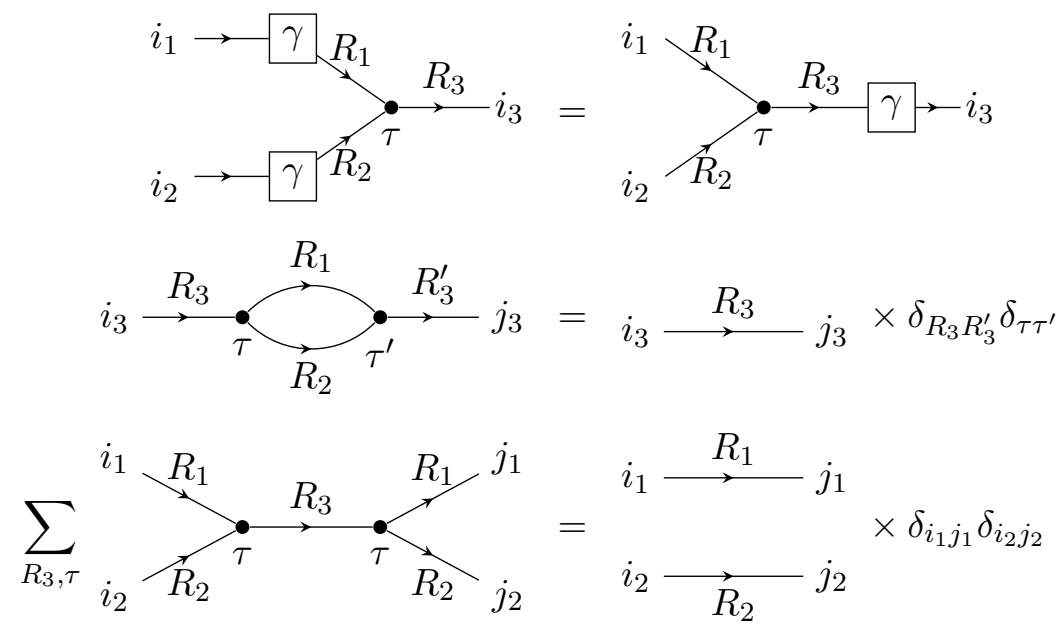

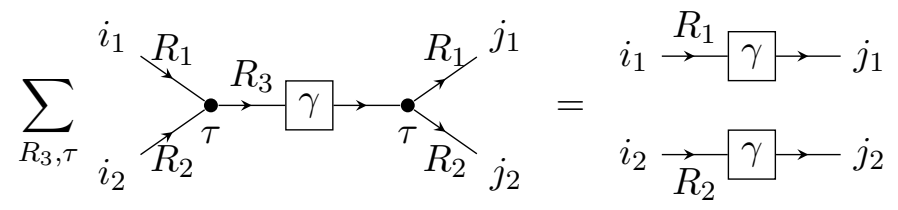

The following lemma is useful in the text.

Lemma 1. The following relation holds:

$$
\begin{gathered}
\sum_{i_{l}, j_{l}} C_{i_{1}, i_{2} ; i_{3}}^{R_{1}, R_{2} ; R_{3}, \tau_{1}} C_{j_{1}, j_{2} ; j_{3}}^{R_{1}, R_{2} ; R_{3}, \tau_{2}} D_{i_{1} j_{1}}^{R_{1}}\left(\gamma_{1} \sigma_{1} \gamma_{2}\right) D_{i_{2} j_{2}}^{R_{2}}\left(\gamma_{1} \sigma_{2} \gamma_{2}\right) D_{i_{3} j_{3}}^{R_{3}}\left(\gamma_{1} \sigma_{3} \gamma_{2}\right) \\
\quad=\sum_{i_{l}, j_{l}} C_{i_{1}, i_{2} ; i_{3}}^{R_{1}, R_{2} ; R_{3}, \tau_{1}} C_{j_{1}, j_{2} ; j_{3}}^{R_{1}, R_{2} ; R_{3}, \tau_{2}} D_{i_{1} j_{1}}^{R_{1}}\left(\sigma_{1}\right) D_{i_{2} j_{2}}^{R_{2}}\left(\sigma_{2}\right) D_{i_{3} j_{3}}^{R_{3}}\left(\sigma_{3}\right)
\end{gathered}
$$


Proof. We have

$$
\begin{aligned}
& \sum_{i_{l}, j_{l}} C_{i_{1}, i_{2} ; i_{3}}^{R_{1}, R_{2}, R_{3}, \tau_{1}} C_{j_{1}, j_{2} ; j_{3}}^{R_{1}, R_{2} ; R_{3}, \tau_{2}} D_{i_{1} j_{1}}^{R_{1}}\left(\gamma_{1} \sigma_{1} \gamma_{2}\right) D_{i_{2} j_{2}}^{R_{2}}\left(\gamma_{1} \sigma_{2} \gamma_{2}\right) D_{i_{3} j_{3}}^{R_{3}}\left(\gamma_{1} \sigma_{3} \gamma_{2}\right) \\
& =\sum_{a_{l}, b_{l}} D_{a_{1} b_{1}}^{R_{1}}\left(\sigma_{1}\right) D_{a_{2} b_{2}}^{R_{2}}\left(\sigma_{2}\right) D_{a_{3} b_{3}}^{R_{3}}\left(\sigma_{3}\right) \sum_{i_{l}} C_{i_{1}, i_{2} ; i_{3}}^{R_{1}, R_{2} ; R_{3}, \tau_{1}} D_{i_{1} a_{1}}^{R_{1}}\left(\gamma_{1}\right) D_{i_{2} a_{2}}^{R_{2}}\left(\gamma_{1}\right) D_{i_{3} a_{3}}^{R_{3}}\left(\gamma_{1}\right) \\
& \quad \times \sum_{j_{l}} C_{j_{1}, j_{2} ; j_{3}}^{R_{1}, R_{2} ; R_{3}, \tau_{2}} D_{b_{1} j_{1}}^{R_{1}}\left(\gamma_{2}\right) D_{b_{2} j_{2}}^{R_{2}}\left(\gamma_{2}\right) D_{b_{3} j_{3}}^{R_{3}}\left(\gamma_{2}\right) \\
& =\sum_{a_{l}, b_{l}} D_{a_{1} b_{1}}^{R_{1}}\left(\sigma_{1}\right) D_{a_{2} b_{2}}^{R_{2}}\left(\sigma_{2}\right) D_{a_{3} b_{3}}^{R_{3}}\left(\sigma_{3}\right) C_{a_{1}, a_{2} ; a_{3}}^{R_{1}, R_{2} ; R_{3}, \tau_{1}} C_{b_{1}, b_{2} ; b_{3}}^{R_{1}, R_{2} ; R_{3}, \tau_{2}}
\end{aligned}
$$

where we used (A.36).

Integrating three representation matrices, the following relation is useful:

Lemma 2. We have

$$
\sum_{\sigma \in S_{n}} D_{i_{1} j_{1}}^{R_{1}}(\sigma) D_{i_{2} j_{2}}^{R_{2}}(\sigma) D_{i_{3} j_{3}}^{R_{3}}(\sigma)=\frac{n !}{d\left(R_{3}\right)} \sum_{\tau} C_{i_{1}, i_{2} ; i_{3}}^{R_{1}, R_{2} ; R_{3}, \tau} C_{j_{1}, j_{2} ; j_{3}}^{R_{1}, R_{2} ; R_{3}, \tau}
$$

Proof. We use, successively, (A.31) and (A.17) to get:

$$
\begin{aligned}
& \sum_{\sigma \in S_{n}} D_{i_{1} j_{1}}^{R_{1}}(\sigma) D_{i_{2} j_{2}}^{R_{2}}(\sigma) D_{i_{3} j_{3}}^{R_{3}}(\sigma)=\sum_{\sigma}\left[\sum_{R, \tau} \sum_{a, b} C_{i_{1}, i_{2} ; a}^{R_{1}, R_{2} ; R, \tau} C_{j_{1}, j_{2} ; b}^{R_{1}, R_{2} ; R, \tau} D_{a b}^{R}(\sigma)\right] D_{i_{3} j_{3}}^{R_{3}}(\sigma) \\
& \quad=\frac{n !}{d\left(R_{3}\right)} \sum_{R, \tau} \sum_{a, b} C_{i_{1}, i_{2} ; a}^{R_{1}, R_{2} ; R, \tau} C_{j_{1}, j_{2} ; b}^{R_{1}, R_{2} ; R, \tau} \delta_{R R_{3}} \delta_{a i_{3}} \delta_{b j_{3}}
\end{aligned}
$$

summing over $R, a$ and $b$ achieves the result.

We can illustrate Lemma 2 in the following way:

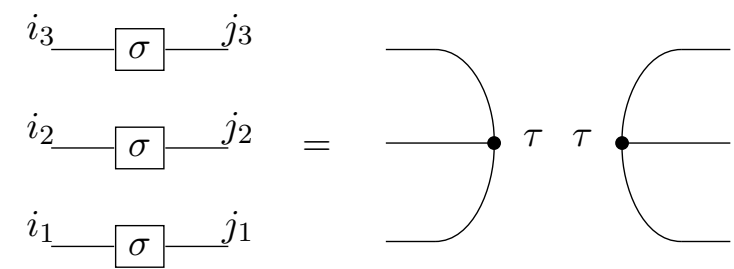

Note that we have defined the Clebsch-Gordan coefficients in terms of inner products between states in $V_{R_{1}} \otimes V_{R_{2}}$ transforming as $V_{R_{3}}$ under the diagonal action of $S_{n}$. We could also have defined them in terms of the states in $V_{R_{1}} \otimes V_{R_{2}} \otimes V_{R_{3}}$ which transform as the trivial representation $[n]$ of the diagonal $S_{3}$. If we use the latter formulation the Clebsch's

$$
\tilde{C}_{i_{1}, i_{2}, i_{3}}^{R_{1}, R_{2}, R_{3} ; \tau}=\left\langle R_{1}, i_{1}, R_{2}, i_{2}, R_{3}, i_{3} \mid[n], \tau\right\rangle
$$

will appear on the r.h.s. of Lemma 2 without the $1 / d\left(R_{3}\right)$ factor, so that

$$
\tilde{C}_{i_{1}, i_{2}, i_{3}}^{R_{1}, R_{2}, R_{3} ; \tau}=\frac{1}{\sqrt{d\left(R_{3}\right)}} C_{i_{1}, i_{2} ; i_{3}}^{R_{1}, R_{2} ; R_{3}, \tau}
$$




\section{A.3 Projectors for irreducible representations}

We discuss some properties of projectors in the group algebra of $\mathbb{C}\left(S_{n}\right)$, which we use in the text. For every irreducible representation $R$ of $\mathbb{C}\left(S_{n}\right)$ we have the characters $\chi^{R}(\sigma)$ which are used to define projectors $P_{R}$

$$
P_{R}=\frac{d(R)}{n !} \sum_{\sigma \in S_{n}} \chi^{R}(\sigma) \sigma
$$

Using character orthogonality we verify that

$$
P_{R} P_{S}=\delta_{R, S} P_{R}
$$

Consider a general representation $W$ of $S_{n}$ which has a decomposition into irreducible representations

$$
W=\bigoplus_{R \vdash n} V_{R} \otimes V_{R}^{\mathrm{m}}
$$

$V_{R}$ are irreducible representation spaces and $V_{R}^{\mathrm{m}}$ are multiplicity spaces of dimension $m_{W}^{R}$. Taking the trace

$$
\begin{aligned}
\operatorname{tr}_{W}\left(P_{S}\right) & =\sum_{R \vdash n} \operatorname{tr}_{V_{R} \otimes V_{R}^{\mathrm{m}}}\left(P_{S} \otimes 1\right) \\
& =\sum_{R} m_{W}^{R} \operatorname{tr}_{V_{R}}\left(P_{S}\right)=\sum_{R} d(R) m_{W}^{R} \delta_{R S} \\
& =m_{W}^{S} d(S)
\end{aligned}
$$

We used

$$
\operatorname{tr}_{V_{R}}\left(P_{S}\right)=\sum_{\sigma \in S_{n}} \frac{d(S)}{n !} \chi^{S}(\sigma) \chi^{R}(\sigma)=d(S) \delta_{R, S}
$$

which is an application of the orthogonality of characters. For the case where $R$ is the trivial representation $R_{0}=[n], \chi^{R_{0}}(\sigma)=1$ and

$$
P_{R_{0}}=\frac{1}{n !} \sum_{\sigma \in S_{n}} \sigma
$$

is the projection on the invariant space of $W$.

\section{B The permutation centralizer algebra $\mathcal{K}(n)$}

\section{B.1 Basis of $\mathcal{K}(n)$}

The semi-simple algebra $\mathcal{K}(n)$ is defined in terms of permutation equivalences in $\mathbb{C}\left(S_{n}\right) \otimes$ $\mathbb{C}\left(S_{n}\right) \otimes \mathbb{C}\left(S_{n}\right)$ (double coset description) or in $\mathbb{C}\left(S_{n}\right) \otimes \mathbb{C}\left(S_{n}\right)$ (centralizer description). By Fourier transforming from the permutation basis of $\mathbb{C}\left(S_{n}\right)$ to the representation basis $Q_{i j}^{R}$, we have Fourier bases for $\mathcal{K}(n)$ in either formulation. In this appendix, we prove that the $Q$-basis elements in each formalism are indeed invariant under the appropriate equivalence relations, that they multiply like matrices (thus giving the WA decomposition) and that they are orthogonal with respect to the non-degenerate bilinear pairing. 
$\boldsymbol{Q}$-Basis of $\mathcal{K}(\boldsymbol{n})$. We start by checking the invariance of the basis $Q_{\tau_{1}, \tau_{2}}^{R, S, T}$ (3.19) under the diagonal action:

$$
\begin{aligned}
(\gamma & \otimes \gamma) \cdot Q_{\tau_{1}, \tau_{2}}^{R, S T} \cdot\left(\gamma^{-1} \otimes \gamma^{-1}\right) \\
= & \kappa_{R, S} \sum_{\sigma_{1}, \sigma_{2} \in S_{n}} \sum_{i_{1}, i_{2}, i_{3}, j_{1}, j_{2}} C_{i_{1}, i_{2} ; i_{3}}^{R, S ; T, \tau_{1}} C_{j_{1}, j_{2} ; i_{3}}^{R, S ; T, \tau_{2}} D_{i_{1} j_{1}}^{R}\left(\sigma_{1}\right) D_{i_{2} j_{2}}^{S}\left(\sigma_{2}\right) \gamma \sigma_{1} \gamma^{-1} \otimes \gamma \sigma_{2} \gamma^{-1} \\
= & \kappa_{R, S} \sum_{\sigma_{1}, \sigma_{2} \in S_{n}} \sum_{i_{1}, i_{2}, i_{3}, j_{1}, j_{2}} C_{i_{1}, i_{2} ; i_{3}}^{R, S ; T, \tau_{1}} C_{j_{1}, j_{2} ; i_{3}}^{R, S ; T, \tau_{2}} D_{i_{1} j_{1}}^{R}\left(\gamma^{-1} \sigma_{1} \gamma\right) D_{i_{2} j_{2}}^{S}\left(\gamma^{-1} \sigma_{2} \gamma\right) \sigma_{1} \otimes \sigma_{2} \\
= & \kappa_{R, S} \sum_{\sigma_{1}, \sigma_{2} \in S_{n}} \sum_{i_{1}, i_{2}, i_{3}, j_{1}, j_{2}} \sum_{a_{l}, b_{l}} C_{i_{1}, i_{2} ; i_{3}}^{R, S ; T, \tau_{1}} C_{j_{1}, j_{2} ; i_{3}}^{R, S ; T, \tau_{2}} D_{i_{1} a_{1}}^{R}\left(\gamma^{-1}\right) D_{a_{1} b_{1}}^{R}\left(\sigma_{1}\right) D_{b_{1} j_{1}}^{R}(\gamma) \\
& \times D_{i_{2} a_{2}}^{S}\left(\gamma^{-1}\right) D_{a_{2} b_{2}}^{S}\left(\sigma_{2}\right) D_{b_{2} j_{2}}^{S}(\gamma) \sigma_{1} \otimes \sigma_{2}
\end{aligned}
$$

Using (A.32) of appendix A.2, we get

$$
\begin{aligned}
(\gamma \otimes & \gamma) \cdot Q_{\tau_{1}, \tau_{2}}^{R, S T} \cdot\left(\gamma^{-1} \otimes \gamma^{-1}\right) \\
= & \kappa_{R, S} \sum_{\sigma_{1}, \sigma_{2} \in S_{n}} \sum_{a_{l}, b_{l}, i_{3}}\left[\sum_{i_{1}, i_{2}} D_{a_{1} i_{1}}^{R}(\gamma) D_{a_{2} i_{2}}^{S}(\gamma) C_{i_{1}, i_{2} ; i_{3}}^{R, S ; T, \tau_{1}}\right]\left[\sum_{j_{1}, j_{2}} D_{b_{1} j_{1}}^{R}(\gamma) D_{b_{2} j_{2}}^{S}(\gamma) C_{j_{1}, j_{2} ; i_{3}}^{R, S ; T, \tau_{2}}\right] \\
& \times D_{a_{1} b_{1}}^{R}\left(\sigma_{1}\right) D_{a_{2} b_{2}}^{S}\left(\sigma_{2}\right) \sigma_{1} \otimes \sigma_{2} \\
= & \kappa_{R, S} \sum_{\sigma_{1}, \sigma_{2} \in S_{n}} \sum_{a_{l}, b_{l}, i_{3}}\left[\sum_{l_{1}} C_{a_{1}, a_{2} ; l_{1}}^{R, S ; T, \tau_{1}} D_{l_{1} i_{3}}^{T}(\gamma)\right]\left[\sum_{l_{2}} C_{b_{1}, b_{2} ; l_{2}}^{R, S ; T, \tau_{2}} D_{l_{2} i_{3}}^{T}(\gamma)\right] \\
& \times D_{a_{1} b_{1}}^{R}\left(\sigma_{1}\right) D_{a_{2} b_{2}}^{S}\left(\sigma_{2}\right) \sigma_{1} \otimes \sigma_{2} \\
= & \kappa_{R, S} \sum_{\sigma_{1}, \sigma_{2} \in S_{n}} \sum_{a_{1}, a_{2}, b_{1}, b_{2}, l} C_{a_{1}, a_{2} ; l}^{R ; T} C_{b_{1}, b_{2} ; l}^{R ; T} D_{a_{1} b_{1}}^{R}\left(\sigma_{1}\right) D_{a_{2} b_{2}}^{S}\left(\sigma_{2}\right) \sigma_{1} \otimes \sigma_{2} \\
= & Q_{\tau_{1}, \tau_{2}}^{R, T}
\end{aligned}
$$

where the factor $\sum_{i_{3}} D_{l_{1} i_{3}}^{T}(\gamma) D_{l_{2} i_{3}}^{T}(\gamma)$ evaluates using (A.3).

We prove now that $Q_{\tau_{1}, \tau_{2}}^{R, S}$ 's multiply like matrices:

$$
\begin{aligned}
& Q_{\tau_{1}, \tau_{2}}^{R, S, T} Q_{\tau_{2}^{\prime}, \tau_{3}}^{R^{\prime}, S^{\prime}, T^{\prime}}=\kappa_{R, S} \kappa_{R^{\prime}, S^{\prime}} \sum_{\sigma_{i}, \sigma_{i}^{\prime} \in S_{n}} \sum_{i_{l}, j_{m}, i_{l}^{\prime}, j_{m}^{\prime}} C_{i_{1}, i_{2} ; i_{3}}^{R, S ; T, \tau_{1}} C_{j_{1}, j_{2} ; i_{3}}^{R, S ; T, \tau_{2}} D_{i_{1} j_{1}}^{R}\left(\sigma_{1}\right) D_{i_{2} j_{2}}^{S}\left(\sigma_{2}\right) \\
& \times C_{i_{1}^{\prime}, i_{2}^{\prime} ; i_{3}^{\prime}}^{R^{\prime}, S^{\prime} ; T_{2}^{\prime}, \tau_{2}^{\prime}} C_{j_{1}^{\prime}, j_{2}^{\prime} ; i_{3}^{\prime}}^{R^{\prime}, S^{\prime} ; \tau_{3}^{\prime}} D_{i_{1}^{\prime} j_{1}^{\prime}}^{R^{\prime}}\left(\sigma_{1}^{\prime}\right) D_{i_{2}^{\prime} j_{2}^{\prime}}^{S^{\prime}}\left(\sigma_{2}^{\prime}\right) \sigma_{1} \sigma_{1}^{\prime} \otimes \sigma_{2} \sigma_{2}^{\prime} \\
& =\kappa_{R, S} \kappa_{R^{\prime}, S^{\prime}} \sum_{\sigma_{i}, \sigma_{i}^{\prime} \in S_{n}} \sum_{i_{l}, j_{m}, i_{l}^{\prime}, j_{m}^{\prime}} C_{i_{1}, i_{2} ; i_{3}}^{R, S ; T, \tau_{1}} C_{j_{1}, j_{2} ; i_{3}}^{R, ; T, \tau_{2}} D_{i_{1} j_{1}}^{R}\left(\sigma_{1} \sigma_{1}^{\prime-1}\right) D_{i_{2} j_{2}}^{S}\left(\sigma_{2} \sigma_{2}^{\prime-1}\right) \\
& \times C_{i_{1}^{\prime}, i_{2}^{\prime} ; i_{3}^{\prime}, T^{\prime}, \tau_{2}^{\prime}}^{R^{\prime}} C_{j_{1}^{\prime}, j_{2}^{\prime} ; i_{3}^{\prime}, \tau_{3}^{\prime}, \tau_{3}}^{R^{\prime}} D_{i_{1}^{\prime} j_{1}^{\prime}}^{R^{\prime}}\left(\sigma_{1}^{\prime}\right) D_{i_{2}^{\prime} j_{2}^{\prime}}^{S^{\prime}}\left(\sigma_{2}^{\prime}\right) \sigma_{1} \otimes \sigma_{2} \\
& =\kappa_{R, S}^{2} \frac{(n !)^{2}}{d(R) d(S)} \delta_{R R^{\prime}} \delta_{S S^{\prime}} \sum_{\sigma_{i} \in S_{n}} \sum_{i_{1}, i_{2}, i_{3}, i_{3}^{\prime}, a, b} C_{i_{1}, i_{2} ; i_{3}}^{R, S ; T, \tau_{1}} C_{a, b ; i_{3}^{\prime}}^{R, S ; T^{\prime}, \tau_{3}} D_{i_{1} a}^{R}\left(\sigma_{1}\right) D_{i_{2} b}^{S}\left(\sigma_{2}\right) \sigma_{1} \otimes \sigma_{2} \\
& \times\left[\sum_{j_{1}, j_{2}} C_{j_{1}, j_{2} ; i_{3}^{\prime}}^{R, ; T_{2}^{\prime}} C_{j_{1}, j_{2} ; i_{3}}^{R, S ; T, \tau_{2}}\right] \\
& =\kappa_{R, S} \delta_{R R^{\prime}} \delta_{S S^{\prime}} \delta_{T T^{\prime}} \delta_{\tau_{2} \tau_{2}^{\prime}} \\
& \times \sum_{\tau_{2}} \sum_{\sigma_{i} \in S_{n}} \sum_{i_{1}, i_{2}, i_{3}, i_{3}^{\prime}, a, b} \delta_{i_{3} i_{3}^{\prime}} C_{i_{1}, i_{2} ; i_{3}}^{R, S ; T, \tau_{1}} C_{a, b ; i_{3}^{\prime}}^{R, S ; T^{\prime}, \tau_{3}} D_{i_{1} a}^{R}\left(\sigma_{1}\right) D_{i_{2} b}^{S}\left(\sigma_{2}\right) \sigma_{1} \otimes \sigma_{2} \\
& =\delta_{R R^{\prime}} \delta_{S S^{\prime}} \delta_{T T^{\prime}} \delta_{\tau_{2} \tau_{2}^{\prime}} Q_{\tau_{1}, \tau_{3}}^{R, S, T}
\end{aligned}
$$

where we used the orthogonality relations (A.17) and (A.33). 
Next, we evaluate the pairing between two basis elements $Q$ 's and check that there are orthogonal:

$$
\begin{aligned}
\delta_{2}\left(Q_{\tau_{1}, \tau_{1}^{\prime}}^{R, S T} ; Q_{\tau_{2}, \tau_{2}^{\prime}}^{R^{\prime}, S^{\prime}, T^{\prime}}\right)= & \kappa_{R, S}^{2} \sum_{\sigma_{i}, \gamma_{i}, \gamma_{i}^{\prime} \in S_{n}} \sum_{i_{l}, j_{l}, i_{l}^{\prime}, j_{l}^{\prime}} C_{i_{1}, i_{2} ; i_{3}}^{R, S ; T, \tau_{1}} C_{j_{1}, j_{2} ; i_{3}}^{R, S ; T} C_{i_{1}^{\prime}, i_{2}^{\prime} ; i_{3}^{\prime}}^{R^{\prime}, T^{\prime} ; T^{\prime}, \tau_{2}} C_{j_{1}^{\prime}, j_{2}^{\prime} ; i_{3}^{\prime}}^{R^{\prime}, S^{\prime} ; T^{\prime}, \tau_{2}^{\prime}} \\
& \times D_{i_{1} j_{1}}^{R}\left(\sigma_{1}\right) D_{i_{2} j_{2}}^{S}\left(\sigma_{2}\right) D_{i_{1}^{\prime} j_{1}^{\prime}}^{R^{\prime}}\left(\sigma_{1}\right) D_{i_{2}^{\prime} j_{2}^{\prime}}^{S^{\prime}}\left(\sigma_{2}\right) \\
= & \kappa_{R, S}^{2} \sum_{i_{l}, j_{l}, i_{l}^{\prime}, j_{l}^{\prime}} C_{i_{1}, i_{2} ; i_{3}}^{R, \tau_{1}} C_{j_{1}, j_{2} ; i_{3}}^{R, S ; T, \tau_{1}^{\prime}} C_{i_{1}^{\prime}, i_{2}^{\prime} ; i_{3}^{\prime}}^{R^{\prime}, S^{\prime}, T_{2}^{\prime}, \tau_{2}} C_{j_{1}^{\prime}, j_{2}^{\prime} ; i_{3}^{\prime}}^{R^{\prime}, \tau_{2}^{\prime} ; \tau_{2}^{\prime}} \\
& \times \frac{(n !)^{2}}{d(R) d(S)} \delta_{R R^{\prime}} \delta_{S S^{\prime}} \delta_{i_{1} i_{1}^{\prime}} \delta_{i_{2} i_{2}^{\prime}} \delta_{j_{1} j_{1}^{\prime}} \delta_{j_{2} j_{2}^{\prime}} \\
= & \kappa_{R, S} \delta_{R R^{\prime}} \delta_{S S^{\prime}} \sum_{i_{l}, j_{l}, i_{3}^{\prime}, j_{l}^{\prime}} C_{i_{1}, i_{2} ; i_{3}}^{R, S ; T, \tau_{1}} C_{i_{1}, i_{2} ; i_{3}^{\prime}}^{R, S ; T^{\prime}, \tau_{2}} C_{j_{1}, j_{2} ; i_{3}}^{R, S ; T, \tau_{1}^{\prime}} C_{j_{1}, j_{2} ; i_{3}^{\prime}}^{R, S ; T^{\prime}, \tau_{2}^{\prime}} \\
= & \kappa_{R, S} d(T) \delta_{R R^{\prime}} \delta_{S S^{\prime}} \delta_{T T^{\prime}} \delta_{\tau_{1} \tau_{2}} \delta_{\tau_{1}^{\prime} \tau_{2}^{\prime}}
\end{aligned}
$$

which shows that according to the normalization that we are using the matrices $Q$ are orthogonal but not normalized.

$\boldsymbol{Q}_{\text {un }}$-basis of $\mathcal{K}_{\text {un }}(\boldsymbol{n})$. We now give few properties of the $Q_{\text {un }}$-basis (3.43). We will skip steps since the derivations are similar to the above case.

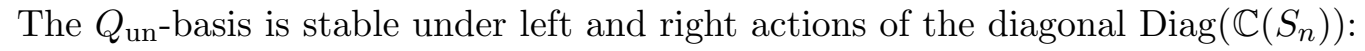

$$
\begin{aligned}
& \left(\gamma_{1}^{\otimes 3}\right) \cdot Q_{\mathrm{un} ; \tau_{1}, \tau_{2}}^{R, S, T} \cdot\left(\gamma_{2}^{\otimes 3}\right)= \\
& =\kappa_{R, S, T} \sum_{\sigma_{i} \in S_{n}} \sum_{i_{l}, j_{l}} \sum_{a_{l}, b_{l}} C_{i_{1}, i_{2} ; i_{3}}^{R, S ; T, \tau_{1}} C_{j_{1}, j_{2} ; i_{3}}^{R, S ; T, \tau_{2}} D_{i_{1} a_{1}}^{R}\left(\gamma_{1}^{-1}\right) D_{a_{1} b_{1}}^{R}\left(\sigma_{1}\right) D_{b_{1} j_{1}}^{R}\left(\gamma_{2}^{-1}\right) \\
& \quad \times D_{i_{2} a_{2}}^{S}\left(\gamma_{1}^{-1}\right) D_{a_{2} b_{2}}^{S}\left(\sigma_{2}\right) D_{b_{2} j_{2}}^{S}\left(\gamma_{2}^{-1}\right) D_{i_{3} a_{3}}^{T}\left(\gamma_{1}^{-1}\right) D_{a_{3} b_{3}}^{T}\left(\sigma_{3}\right) D_{b_{3} j_{3}}^{T}\left(\gamma_{2}^{-1}\right) \sigma_{1} \otimes \sigma_{2} \otimes \sigma_{3}
\end{aligned}
$$

We use (A.32) and (A.3) (of appendix A.2) to reduce the above to $Q_{\mathrm{un} ; \tau_{1}, \tau_{2}}^{R, S, T}$.

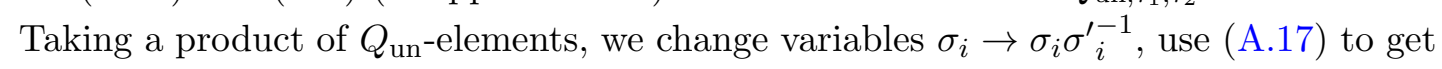

$$
\begin{aligned}
& Q_{\mathrm{un} ; \tau_{1}, \tau_{2}}^{R, S,} Q_{\mathrm{un} ; \tau_{2}^{\prime}, \tau_{3}}^{R^{\prime}, T^{\prime}, T^{\prime}} \\
& =\kappa_{R, S, T} \kappa_{R^{\prime}, S^{\prime}, T^{\prime}} \sum_{\sigma_{l}, \sigma_{l}^{\prime}} \sum_{i_{l}, i_{l}^{\prime}, j_{l}, j_{l}^{\prime}} C_{i_{1}, i_{2} ; i_{3}}^{R, S ; T} C_{j_{1}, \tau_{2} ; j_{3}}^{R, S ; T, \tau_{2}} C_{i_{1}^{\prime}, i_{2}^{\prime} ; i_{3}^{\prime}}^{R^{\prime}, S^{\prime} ; T^{\prime}, \tau_{2}^{\prime}} C_{j_{1}^{\prime}, j_{2}^{\prime} ; j_{3}^{\prime}}^{R^{\prime}, S^{\prime}, T_{3}^{\prime}, \tau_{3}} \\
& \quad \times D_{i_{1}, j_{1}}^{R}\left(\sigma_{1} \sigma_{1}^{\prime-1}\right) D_{i_{2}, j_{2}}^{S}\left(\sigma_{2} \sigma_{2}^{\prime-1}\right) D_{i_{3}, j_{3}}^{T}\left(\sigma_{3} \sigma_{3}^{\prime-1}\right) D_{i_{1}^{\prime}, j_{1}^{\prime}}^{R^{\prime}}\left(\sigma_{1}^{\prime}\right) D_{i_{2}^{\prime}, j_{2}^{\prime}}^{S^{\prime}}\left(\sigma_{2}^{\prime}\right) D_{i_{3}^{\prime}, j_{3}^{\prime}}^{T^{\prime}}\left(\sigma_{3}^{\prime}\right) \sigma_{1} \otimes \sigma_{2} \otimes \sigma_{3} \\
& =\delta_{R R^{\prime}} \delta_{S S^{\prime}} \delta_{T T^{\prime}} \delta_{\tau_{2} \tau_{2}^{\prime}} Q_{\mathrm{un} ; \tau_{1}, \tau_{3}}^{R, S, T}
\end{aligned}
$$

which shows that $Q_{\text {un }}$ multiply like matrices.

Computing the pairing of two elements of the $Q_{\mathrm{un}}$-basis, we obtain

$$
\begin{aligned}
\delta_{3}\left(Q_{\mathrm{un} ; \tau_{1}, \tau_{2}}^{R, S, T} Q_{\mathrm{un} ; \tau_{2}^{\prime}, \tau_{3}}^{R^{\prime}, S^{\prime}, T^{\prime}}\right) & =\kappa_{R, S, T} \delta_{R R^{\prime}} \delta_{S S^{\prime}} \delta_{T T^{\prime}} \sum_{i_{l}, j_{l}} C_{i_{1}, i_{2} ; i_{3}}^{R, S T T \tau_{1}} C_{i_{1}, i_{2} ; i_{3}}^{R, ; T, \tau_{2}^{\prime}} C_{j_{1}, j_{2} ; j_{3}}^{R, S T T, \tau_{2}} C_{j_{1}, j_{2} ; j_{3}}^{R, S ; T, \tau_{3}} \\
& =\kappa_{R, S, T} d(T)^{2} \delta_{R R^{\prime}} \delta_{S S^{\prime}} \delta_{T T^{\prime}} \delta_{\tau_{1} \tau_{2}^{\prime}} \delta_{\tau_{2} \tau_{3}}
\end{aligned}
$$




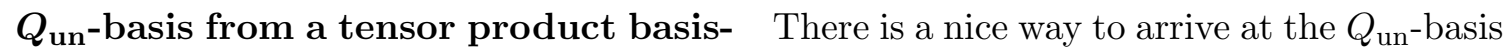
for $\mathcal{K}(n)$ by starting from the representation theoretic Fourier basis for the tensor product $\mathbb{C}\left(S_{n}\right) \otimes \mathbb{C}\left(S_{n}\right) \otimes \mathbb{C}\left(S_{n}\right)$.

Consider in the group algebra $\mathbb{C}\left(S_{n}\right)$, the elements

$$
Q_{i j}^{R}=\frac{\kappa_{R}}{n !} \sum_{\sigma \in S_{n}} D_{i j}^{R}(\sigma) \sigma
$$

with $\kappa_{R}$ is a normalization factor that we will fix after introducing the pairing. $R$ is any irreducible representation of $S_{n}$, parametrised by partitions of $n$ or Young diagrams with $n$ boxes. The indices $i, j$ run over an orthonormal basis set for the representation. The number of these $Q_{i j}^{R}$ is equal to $n$ ! thanks to a standard group theory identity

$$
\sum_{R \vdash n}(d(R))^{2}=n !
$$

These $Q_{i j}^{R}$ form a representation theoretic Fourier basis for $\mathbb{C}\left(S_{n}\right)$.

We have the following important properties:

$$
\tau Q_{i j}^{R}=\sum_{l} D_{l i}^{R}(\tau) Q_{l j}^{R}, \quad Q_{i j}^{R} \tau=\sum_{l} Q_{i l}^{R} D_{j l}^{R}(\tau)
$$

There is a pairing on $\mathbb{C}\left(S_{n}\right)^{d}$, such that

$$
\delta\left(\sigma_{1} \otimes \cdots \otimes \sigma_{d} ; \sigma_{1}^{\prime} \otimes \cdots \otimes \sigma_{d}^{\prime}\right)=\delta\left(\sigma_{1} \sigma_{1}^{\prime-1}\right) \ldots \delta\left(\sigma_{d}^{-1} \sigma_{d}^{\prime-1}\right)
$$

and such that

$$
\delta\left(Q_{i j}^{R} ; Q_{i^{\prime} j^{\prime}}^{R^{\prime}}\right)=\frac{\kappa_{R} \kappa_{R^{\prime}}}{(n !)^{2}} \sum_{\sigma} D_{i j}^{R}(\sigma) D_{i^{\prime} j^{\prime}}^{R^{\prime}}(\sigma)=\frac{\kappa_{R}^{2}}{n ! d(R)} \delta_{R R^{\prime}} \delta_{i i^{\prime}} \delta_{j j^{\prime}}=\delta_{R R^{\prime}} \delta_{i i^{\prime}} \delta_{j j^{\prime}}
$$

with $\kappa_{R}^{2}=n ! d(R)$. Then

$$
\delta\left(Q_{i_{1} j_{1}}^{R_{1}} \otimes \cdots \otimes Q_{i_{d} j_{d}}^{R_{d}} ; Q_{i_{1}^{\prime} j_{1}^{\prime}}^{R_{1}^{\prime}} \otimes \cdots \otimes Q_{i_{d}^{\prime} j_{d}^{\prime}}^{R_{1}^{\prime}}\right)=\delta_{R_{1} R_{1}^{\prime}} \delta_{i_{1} i_{1}^{\prime}} \delta_{j_{1} j_{1}^{\prime}} \ldots \delta_{R_{d} R_{d}^{\prime}} \delta_{i_{d} i_{d}^{\prime}} \delta_{j_{d} j_{d}^{\prime}}
$$

Hence, the basis $\left\{Q_{i_{1} j_{1}}^{R_{1}} \otimes \cdots \otimes Q_{i_{d} j_{d}}^{R_{d}}\right\}$ is an orthonormal (Fourier-like) basis for $\mathbb{C}\left(S_{n}\right)^{d}$.

Let us restrict now to rank $d=3$ (the following extends to any $d$ easily). Consider $\rho_{L}\left(\tau_{1}\right)$ and $\rho_{R}\left(\tau_{2}\right)$ the left and right diagonal action on the tensor product $\mathbb{C}\left(S_{n}\right)^{3}$. Then we write:

$$
\begin{aligned}
& \sum_{\sigma_{1}, \sigma_{2}} \rho_{L}\left(\sigma_{1}\right) \rho_{R}\left(\sigma_{2}\right) Q_{i_{1} j_{1}}^{R_{1}} \otimes Q_{i_{2} j_{2}}^{R_{2}} \otimes Q_{i_{3} j_{3}}^{R_{3}}=\sum_{\sigma_{1}, \sigma_{2}} \sigma_{1} Q_{i_{1} j_{1}}^{R_{1}} \sigma_{2} \otimes \sigma_{1} Q_{i_{2} j_{2}}^{R_{2}} \sigma_{2} \otimes \sigma_{1} Q_{i_{3} j_{3}}^{R_{3}} \sigma_{2} \\
& =\sum_{\sigma_{1}, \sigma_{2}} \sum_{p_{l}, q_{l}} D_{p_{1} i_{1}}^{R_{1}}\left(\sigma_{1}\right) Q_{p_{1} q_{1}}^{R_{1}} D_{j_{1} q_{1}}^{R_{1}}\left(\sigma_{2}\right) \otimes D_{p_{2} i_{2}}^{R_{2}}\left(\sigma_{1}\right) Q_{p_{2} q_{2}}^{R_{2}} D_{j_{2} q_{2}}^{R_{2}}\left(\sigma_{2}\right) \otimes D_{p_{3} i_{3}}^{R_{3}}\left(\sigma_{1}\right) Q_{p_{3} q_{3}}^{R_{3}} D_{j_{3} q_{3}}^{R_{3}}\left(\sigma_{2}\right) \\
& =\frac{(n !)^{2}}{d\left(R_{3}\right)^{2}} \sum_{p_{l}, q_{l}} \sum_{\tau, \tau^{\prime}} C_{p_{1}, p_{2} ; p_{3}}^{R_{1}, R_{2} ; R_{3}, \tau} C_{i_{1}, i_{2} ; i_{3}}^{R_{1}, R_{2} ; R_{3}, \tau} C_{j_{1}, j_{2} ; j_{3}}^{R_{1}, R_{2} ; R_{3}, \tau^{\prime}} C_{q_{1}, q_{2} ; q_{3}}^{R_{1}, R_{2} ; R_{3}, \tau^{\prime}} Q_{p_{1} q_{1}}^{R_{1}} \otimes Q_{p_{2} q_{2}}^{R_{2}} \otimes Q_{p_{3} q_{3}}^{R_{3}}
\end{aligned}
$$


where we used (B.10) and Lemma 2 for summing $\sigma_{1}, \sigma_{2}$, (in appendix A.2). Then we couple the last result with two CG's, and use (A.33), in such a way to have:

$$
\begin{aligned}
\sum_{i_{l}, j_{l}} & C_{i_{1}, i_{2} ; i_{3}}^{R_{1}, R_{3}, R_{3}, \tau} C_{j_{1}, j_{2} ; j_{3}}^{R_{1}, R_{2} ; R_{3}, \tau^{\prime}} \sum_{\sigma_{1}, \sigma_{2}} \rho_{L}\left(\sigma_{1}\right) \rho_{R}\left(\sigma_{2}\right) Q_{i_{1} j_{1}}^{R_{1}} \otimes Q_{i_{2} j_{2}}^{R_{2}} \otimes Q_{i_{3} j_{3}}^{R_{3}} \\
= & \frac{(n !)^{2}}{d\left(R_{3}\right)^{2}} \sum_{p_{l}, q_{l}} \sum_{\varrho, \varrho^{\prime}} \sum_{i_{l}, j_{l}} C_{i_{1}, i_{2} ; i_{3}}^{R_{1}, R_{2} ; R_{3}, \tau} C_{i_{1}, i_{2} ; i_{3}}^{R_{1}, R_{2} ; R_{3}, \varrho} C_{j_{1}, j_{2} ; j_{3}}^{R_{1}, R_{2} ; R_{3}, \tau^{\prime}} C_{j_{1}, j_{2} ; j_{3}}^{R_{1}, R_{2} ; R_{3}, \varrho^{\prime}} \\
& \times C_{p_{1}, p_{2} ; p_{3}}^{R_{1}, R_{2} ; R_{3}, \varrho} C_{q_{1}, q_{2} ; q_{3}}^{R_{1}, R_{3}, \varrho^{\prime}} Q_{p_{1} q_{1}}^{R_{1}} \otimes Q_{p_{2} q_{2}}^{R_{2}} \otimes Q_{p_{3} q_{3}}^{R_{3}} \\
= & (n !)^{2} \sum_{p_{l}, q_{l}} C_{p_{1}, p_{2} ; p_{3}}^{R_{1}, R_{2} ; R_{3}, \tau} C_{q_{1}, q_{2} ; q_{3}}^{R_{1}, R_{2} ; \tau^{\prime}} Q_{p_{1} q_{1}}^{R_{1}} \otimes Q_{p_{2} q_{2}}^{R_{2}} \otimes Q_{p_{3} q_{3}}^{R_{3}}
\end{aligned}
$$

The last expression matches $Q_{\mathrm{un} ; \tau, \tau^{\prime}}^{R_{1}, R_{2}, R_{3}}$ up to a normalization (3.43).

From

$$
\sum_{i_{l}, j_{l}} C_{i_{1}, i_{2} ; i_{3}}^{R_{1}, R_{2} ; R_{3}, \tau} C_{j_{1}, j_{2} ; j_{3}}^{R_{1}, R_{2} ; R_{3}, \tau^{\prime}} \sum_{\sigma_{1}, \sigma_{2}} \rho_{L}\left(\sigma_{1}\right) \rho_{R}\left(\sigma_{2}\right) Q_{i_{1} j_{1}}^{R_{1}} \otimes Q_{i_{2} j_{2}}^{R_{2}} \otimes Q_{i_{3} j_{3}}^{R_{3}}
$$

we can infer invariance under left and right diagonal action of $Q_{\mathrm{un} ; \tau, \tau^{\prime}}^{R_{1}, R_{2}, R_{3}}$ : fix $\gamma_{1}, \gamma_{2}$,

$$
\rho_{L}\left(\gamma_{1}\right) \rho_{R}\left(\gamma_{2}\right)\left[\sum_{\sigma_{1}, \sigma_{2}} \rho_{L}\left(\sigma_{1}\right) \rho_{R}\left(\sigma_{2}\right)\right]=\sum_{\sigma_{1}, \sigma_{2}} \rho_{L}\left(\gamma_{1} \sigma_{1}\right) \rho_{R}\left(\gamma_{2} \sigma_{1}\right)=\sum_{\sigma_{1}, \sigma_{2}} \rho_{L}\left(\sigma_{1}\right) \rho_{R}\left(\sigma_{2}\right) .
$$

Finally, similar derivations allows one to get $Q_{\tau, \tau^{\prime}}^{R_{1}, R_{2}, R_{3}}(3.19)$ in terms of diagonal adjoint action coupled with CG's.

\section{B.2 Basis of $\mathcal{Z}(\mathcal{K}(n))$}

Overcompleteness of the $z_{R_{1}, R_{2} ; R_{3}}$-basis in $\mathcal{Z}(\mathcal{K}(n))$. Consider the elements

$$
z_{R_{1}, R_{2} ; R_{3}}=\left(z_{R_{1}} \otimes z_{R_{2}}\right) \cdot z_{R_{3}}, \quad z_{R_{1,2}}=\sum_{\sigma} \chi^{R_{1,2}}(\sigma) \sigma, \quad z_{R_{3}}=\sum_{\sigma} \chi^{R_{3}}(\sigma) \sigma \otimes \sigma
$$

which are elements of $\mathcal{Z}(\mathcal{K}(n))$. We evaluate now the overlapping between the basis $P^{R_{1}, R_{2}, R_{3}}$ and the elements $z_{R_{1}^{\prime}, R_{2}^{\prime} ; R_{3}^{\prime}}$ :

$$
\begin{aligned}
\boldsymbol{\delta}_{2}( & \left.P^{R_{1}, R_{2}, R_{3}} ; z_{R_{1}^{\prime}, R_{2}^{\prime} ; R_{3}^{\prime}}\right) \\
= & \sum_{\tau} \sum_{\sigma_{i} \in S_{n}} \chi^{R_{1}^{\prime}}\left(\sigma_{1}\right) \chi^{R_{2}^{\prime}}\left(\sigma_{2}\right) \chi^{R_{3}^{\prime}}\left(\sigma_{3}\right) \boldsymbol{\delta}_{2}\left(Q_{\tau, \tau}^{R_{1}, R_{2}, R_{3}} ; \sigma_{1} \sigma_{3} \otimes \sigma_{2} \sigma_{3}\right) \\
= & \kappa_{R_{1}, R_{2}} \sum_{\tau} \sum_{\sigma_{l}^{\prime}, \sigma_{l} \in S_{n}} \sum_{i_{l}, j_{l}} C_{i_{1}, i_{2} ; i_{3}}^{R_{1}, R_{2} ; R_{3}, \tau} C_{j_{1}, j_{2} ; i_{3}}^{R_{1}, R_{2} ; R_{3}, \tau} \\
& \times D_{i_{1} j_{1}}^{R_{1}}\left(\sigma_{1}^{\prime}\right) D_{i_{2} j_{2}}^{R_{2}}\left(\sigma_{2}^{\prime}\right) \chi^{R_{1}^{\prime}}\left(\sigma_{1}\right) \chi^{R_{2}^{\prime}}\left(\sigma_{2}\right) \chi^{R_{3}^{\prime}}\left(\sigma_{3}\right) \boldsymbol{\delta}_{2}\left(\sigma_{1}^{\prime} \otimes \sigma_{2}^{\prime} ; \sigma_{1} \sigma_{3} \otimes \sigma_{2} \sigma_{3}\right) \\
= & \kappa_{R_{1}, R_{2}} \sum_{\tau} \sum_{\sigma_{l} \in S_{n}} \sum_{i_{l}, j_{l}} C_{i_{1}, i_{2} ; i_{3}}^{R_{1}, R_{2} ; R_{3}, \tau} C_{j_{1}, j_{2} ; i_{3}}^{R_{1}, R_{2} ; R_{3}, \tau} \\
& \times \sum_{a_{l}, b_{l}} D_{i_{1} a_{1}}^{R_{1}}\left(\sigma_{1}\right) D_{a_{1} j_{1}}^{R_{1}}\left(\sigma_{3}\right) D_{i_{2} a_{2}}^{R_{2}}\left(\sigma_{2}\right) D_{a_{2} j_{2}}^{R_{2}}\left(\sigma_{3}\right) D_{b_{1} b_{1}}^{R_{1}^{\prime}}\left(\sigma_{1}\right) D_{b_{2} b_{2}}^{R_{2}^{\prime}}\left(\sigma_{2}\right) D_{b_{3} b_{3}}^{R_{3}^{\prime}}\left(\sigma_{3}\right)
\end{aligned}
$$




$$
\begin{aligned}
= & \kappa_{R_{1}, R_{2}} \frac{(n !)^{2}}{d\left(R_{1}\right) d\left(R_{2}\right)} \delta_{R_{1} R_{1}^{\prime}} \delta_{R_{2} R_{2}^{\prime}} \sum_{\tau} \sum_{i_{l}, j_{l}} \sum_{\sigma} C_{i_{1}, i_{2} ; i_{3}}^{R_{1}, R_{2} ; R_{3}, \tau} C_{j_{1}, j_{2} ; i_{3}}^{R_{1}, R_{2} ; R_{3}, \tau} \\
& \times \sum_{a_{l}, b_{l}} \delta_{i_{1} b_{1}} \delta_{a_{1} b_{1}} \delta_{i_{2} b_{2}} \delta_{a_{2} b_{2}} D_{a_{1} j_{1}}^{R_{1}}(\sigma) D_{a_{2} j_{2}}^{R_{2}}(\sigma) D_{b_{3} b_{3}}^{R_{3}^{\prime}}(\sigma) \\
= & \kappa_{R_{1}, R_{2}} \frac{(n !)^{2}}{d\left(R_{1}\right) d\left(R_{2}\right)} \delta_{R_{1} R_{1}^{\prime}} \delta_{R_{2} R_{2}^{\prime}} \sum_{\tau} \sum_{b_{3}} \sum_{i_{l}, j_{l}} \sum_{\sigma} C_{i_{1}, i_{2} ; i_{3}}^{R_{1}, R_{2} ; R_{3}, \tau} C_{j_{1}, j_{2} ; i_{3}}^{R_{1}, R_{2} ; R_{3}, \tau} \\
& \times D_{i_{1} j_{1}}^{R_{1}}(\sigma) D_{i_{2} j_{2}}^{R_{2}}(\sigma) D_{b_{3} b_{3}}^{R_{3}^{\prime}}(\sigma) \\
= & \delta_{R_{1} R_{1}^{\prime}} \delta_{R_{2} R_{2}^{\prime}} \sum_{\tau} \sum_{b_{3}, l} \sum_{i_{l}} \sum_{\sigma} C_{i_{1}, i_{2} ; i_{3}}^{R_{1}, R_{2} ; R_{3}, \tau} C_{i_{1}, i_{2} ; l}^{R_{1}, R_{2} ; R_{3}, \tau} D_{b_{3} b_{3}}^{R_{3}^{\prime}}(\sigma) D_{l i_{3}}^{R_{3}}(\sigma) \\
= & \delta_{R_{1} R_{1}^{\prime}} \delta_{R_{2} R_{2}^{\prime}} \mathrm{C}\left(R_{1}, R_{2}, R_{3}\right) \sum_{b, l} \sum_{\sigma} D_{b b}^{R_{3}^{\prime}}(\sigma) D_{l l}^{R_{3}}(\sigma) \\
= & n ! \delta_{R_{1} R_{1}^{\prime}} \delta_{R_{2} R_{2}^{\prime}} \delta_{R_{3} R_{3}^{\prime}} \mathrm{C}\left(R_{1}, R_{2}, R_{3}\right)
\end{aligned}
$$

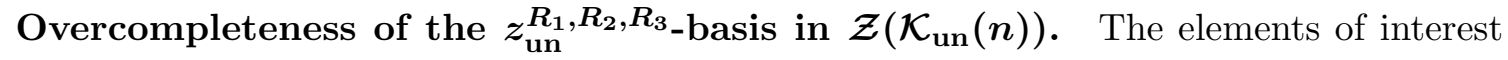
are of the form

$$
z_{\mathrm{un}}^{R_{1}, R_{2}, R_{3}}=z_{R_{1}} \otimes z_{R_{2}} \otimes z_{R_{3}}, \quad z_{R_{i}}=\sum_{\sigma} \chi^{R_{i}}(\sigma) \sigma
$$

which are elements of $\mathcal{Z}(\mathcal{K}(n))$. Note that they are more symmetric in the three indices than the previous central element. We compute the overlap:

$$
\begin{aligned}
\boldsymbol{\delta}_{3} & \left(z_{\mathrm{un}}^{R_{1}, R_{2}, R_{3}} ; P_{\mathrm{un}}^{R_{1}^{\prime}, R_{2}^{\prime}, R_{3}^{\prime}}\right) \\
= & \sum_{\tau} \sum_{\sigma_{i}} \chi^{R_{1}}\left(\sigma_{1}\right) \chi^{R_{2}}\left(\sigma_{2}\right) \chi^{R_{3}}\left(\sigma_{3}\right) \boldsymbol{\delta}_{3}\left(\sigma_{1} \otimes \sigma_{2} \otimes \sigma_{3} ; Q_{\mathrm{un} ; \tau, \tau}^{R_{1}^{\prime}, R_{2}^{\prime}, R_{3}^{\prime}}\right) \\
= & \kappa_{R_{1}^{\prime}, R_{2}^{\prime}, R_{3}^{\prime}} \sum_{\tau} \sum_{\sigma_{l}, \sigma_{l}^{\prime} \in S_{n}} \sum_{i_{l}, j_{l}} C_{i_{1}, i_{2} ; i_{3}}^{R_{1}^{\prime}, R_{2}^{\prime} ; R_{3}^{\prime}, \tau} C_{j_{1}, j_{2} ; j_{3}}^{R_{1}^{\prime}, R_{2}^{\prime} ; R_{3}^{\prime}, \tau} \\
& \times \chi^{R_{1}}\left(\sigma_{1}\right) \chi^{R_{2}}\left(\sigma_{2}\right) \chi^{R_{3}}\left(\sigma_{3}\right) D_{i_{1}, j_{1}}^{R_{1}^{\prime}}\left(\sigma_{1}^{\prime}\right) D_{i_{2}, j_{2}}^{R_{2}^{\prime}}\left(\sigma_{2}^{\prime}\right) D_{i_{3}, j_{3}}^{R_{3}^{\prime}}\left(\sigma_{3}^{\prime}\right) \boldsymbol{\delta}_{3}\left(\sigma_{1} \otimes \sigma_{2} \otimes \sigma_{3} ; \sigma_{1}^{\prime} \otimes \sigma_{2}^{\prime} \otimes \sigma_{3}^{\prime}\right) \\
= & \kappa_{R_{1}^{\prime}, R_{2}^{\prime}, R_{3}^{\prime}} \sum_{\tau} \sum_{\sigma_{l} \in S_{n}} \sum_{i_{l}, j_{l}} C_{i_{1}, i_{2} ; i_{3}}^{R_{1}^{\prime}, R_{3}^{\prime} ; R_{3}^{\prime}, \tau} C_{j_{1}, j_{2} ; j_{3}}^{R_{1}^{\prime}, R_{3}^{\prime}, \tau}, \\
& \times \chi^{R_{1}}\left(\sigma_{1}\right) \chi^{R_{2}}\left(\sigma_{2}\right) \chi^{R_{3}}\left(\sigma_{3}\right) D_{i_{1}, j_{1}}^{R_{1}^{\prime}}\left(\sigma_{1}\right) D_{i_{2}, j_{2}}^{R_{2}^{\prime}}\left(\sigma_{2}\right) D_{i_{3}, j_{3}}^{R_{3}^{\prime}}\left(\sigma_{3}\right) \\
= & \delta_{R_{1} R_{1}^{\prime}} \delta_{R_{2} R_{2}^{\prime}} \delta_{R_{3} R_{3}^{\prime}} \sum_{\tau} \sum_{i_{l}} C_{i_{1}, i_{2} ; i_{3}}^{R_{1}, R_{2} ; R_{3}, \tau} C_{i_{1}, i_{2} ; i_{3}}^{R_{1}, R_{2} ; R_{3}, \tau} \\
= & d\left(R_{3}\right) \mathrm{C}\left(R_{1}, R_{2}, R_{3}\right) \delta_{R_{1} R_{1}^{\prime}} \delta_{R_{2} R_{2}^{\prime}} \delta_{R_{3} R_{3}^{\prime}}
\end{aligned}
$$

That coefficient is not vanishing in general hence $P^{R_{1}, R_{2}, R_{3}}$ admits an expansion in the $z_{R_{1}, R_{2}, R_{3}}$-basis.

\section{Multiplication table of rank $d=3, n=3$ colored tensor graphs}

We list, in this appendix, the multiplication table of the 10 invariants (recall that there are 11 invariants but the row for the identity element $E=\stackrel{\ominus}{\ominus}$ is trivial) of rank $d=3$ at $2 n=6$ 
number of vertices. The index $c=1,2,3$ is a color label of a graph. Define $\check{c}=1,2,3$ such that $\check{c} \neq c$, and $\check{c}=1,2,3$ which can be neither $\check{c}$ nor $c$, we obtain table 5 giving the multiplication between the 10 possible elements.

The table teaches us that the basic products are all commutatives and so is the algebra $\mathcal{K}_{\text {un }}(3)$. Furthermore, some elements admit a or multiple factorizations:

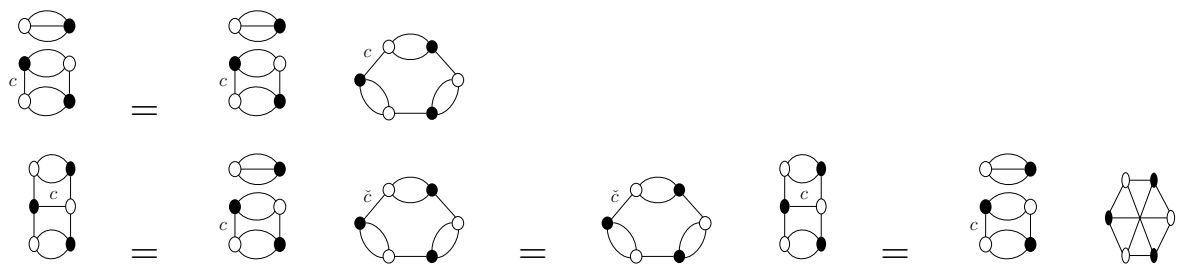

\section{Correlators of tensor observables}

Correlators involves insertions and evaluation of general observables in the Gaussian path integral. We will restrict attention to $d=3$ and consider the Gaussian model

$$
\mathcal{Z}=\int d \Phi d \bar{\Phi} e^{-\frac{1}{2} \sum_{i_{l}} \Phi_{i_{1} i_{2} i_{3}} \bar{\Phi}_{i_{1} i_{2} i_{3}}}
$$

The index $i_{a}$ takes values in $\left\{1 \cdots N_{a}\right\}$, for $a \in\{1,2,3\}$. The propagator or 2-point function is of the form

$$
\left\langle\Phi_{i_{1} i_{2} i_{3}} \bar{\Phi}_{j_{1} j_{2} j_{3}}\right\rangle=\delta_{i_{1} j_{1}} \delta_{i_{2} j_{2}} \delta_{i_{3} j_{3}}
$$

The observables, invariant under $\mathrm{U}(N) \times \mathrm{U}(N) \times \mathrm{U}(N)$, are labelled by permutations $\left(\sigma_{1}, \sigma_{2}, \sigma_{3}\right)$ subject to equivalence $\left(\sigma_{1}, \sigma_{2}, \sigma_{3}\right) \sim\left(\gamma_{1} \sigma_{1} \gamma_{2}, \gamma_{1} \sigma_{2} \gamma_{2}, \gamma_{1} \sigma_{3} \gamma_{2}\right)$. We will write these observables subjected to the equivalence as $\mathcal{O}_{\sigma_{1}, \sigma_{2}, \sigma_{3}}=\mathcal{O}_{\gamma_{1} \sigma_{1} \gamma_{2}, \gamma_{1} \sigma_{2} \gamma_{2}, \gamma_{1} \sigma_{3} \gamma_{2}}$. We recall

$$
\begin{aligned}
\mathcal{O}_{\sigma_{1}, \sigma_{2}, \sigma_{3}}= & \sum_{i_{l}, j_{l}, k_{l}} \Phi_{i_{1} j_{1} k_{1}} \Phi_{i_{2} j_{2} k_{2}} \ldots \Phi_{i_{n} j_{n} k_{n}} \\
& \times \bar{\Phi}_{i_{\sigma_{1}(1)} j_{\sigma_{2}(1)} k_{\sigma_{3}(1)}} \bar{\Phi}_{i_{\sigma_{1}(2)} j_{\sigma_{2}(2)} k_{\sigma_{3}(2)}} \ldots \bar{\Phi}_{i_{\sigma_{1}(n)} j_{\sigma_{2}(n)} k_{\sigma_{3}(n)}}
\end{aligned}
$$

The integral the such operators is given by the Wick theorem

$$
\begin{aligned}
\left\langle\mathcal{O}_{\sigma_{1}, \sigma_{2}, \sigma_{3}}\right\rangle= & \frac{1}{\mathcal{Z}} \int d \Phi d \bar{\Phi} e^{-\frac{1}{2} \sum_{i, j, k} \Phi_{i j k} \bar{\Phi}_{i j k}} \mathcal{O}_{\sigma_{1}, \sigma_{2}, \sigma_{3}} \\
= & \sum_{i_{l}, j_{l}, k_{l}} \sum_{\mu \in S_{n}} \delta_{i_{1} i_{\mu\left(\sigma_{1}(1)\right)}} \delta_{i_{2} i_{\mu\left(\sigma_{1}(2)\right)}} \ldots \delta_{i_{n} i_{\mu\left(\sigma_{1}(n)\right)}} \\
& \times \delta_{j_{1} j_{\mu\left(\sigma_{2}(1)\right)}} \delta_{j_{2} j_{\mu\left(\sigma_{2}(2)\right)}} \ldots \delta_{j_{n} j_{\mu\left(\sigma_{2}(n)\right)}} \delta_{k_{1} k_{\mu\left(\sigma_{3}(1)\right)}} \delta_{k_{2} k_{\mu\left(\sigma_{3}(2)\right)}} \ldots \delta_{k_{n} k_{\mu\left(\sigma_{3}(n)\right)}} \\
= & \sum_{\mu \in S_{n}} N^{\mathbf{c}\left(\mu \sigma_{1}\right)+\mathbf{c}\left(\mu \sigma_{2}\right)+\mathbf{c}\left(\mu \sigma_{3}\right)}
\end{aligned}
$$

where $\mathbf{c}(\alpha)$ is the number of cycles of $\alpha \in S_{n}$. We have used the fact that, given $\sigma \in S_{n}$, $i_{l} \in \llbracket 1, N \rrbracket, l=1, \ldots, n$,

$$
\sum_{i_{l}} \delta_{i_{\sigma(1)}}^{i_{1}} \ldots \delta_{i_{\sigma(n)}}^{i_{n}}=N^{\mathbf{c}(\sigma)}
$$

This allows us to recover (2.10). 


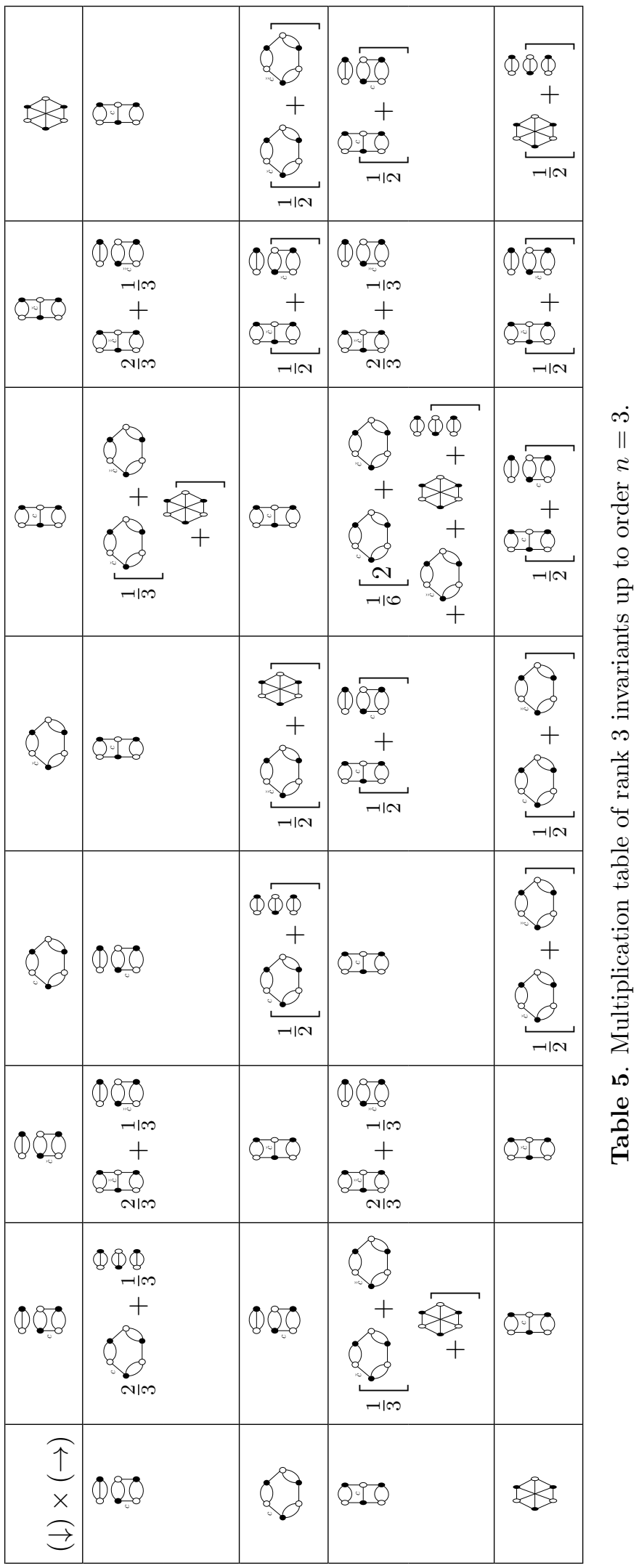


Two point-functions $\left\langle\mathcal{O}_{\sigma_{1}, \sigma_{2}, \sigma_{3}} \overline{\mathcal{O}}_{\tau_{1}, \tau_{2}, \tau_{3}}\right\rangle$ can be also computed in a similar way. We give a summary of appendix $\mathrm{C}$ in [41] (note that we correct a few mistakes appearing this appendix below). We are taking the observables to be "normal ordered" so we only allow contractions to take place between the $\Phi$ 's from the first observable to the $\bar{\Phi}$ 's from the second (parametrized by $\mu_{a}$ ) and between the $\bar{\Phi}$ 's from the first observable to the $\Phi$ 's from the second (parametrized by $\nu_{a}$ ):

$$
\left\langle\mathcal{O}_{\sigma_{1}, \sigma_{2}, \sigma_{3}} \overline{\mathcal{O}}_{\tau_{1}, \tau_{2}, \tau_{3}}\right\rangle=\sum_{\mu \in S_{n}} \sum_{\nu \in S_{n}} \operatorname{tr}_{V_{1}^{\otimes n}}\left(\sigma_{1} \mu \tau_{1}^{-1} \nu\right) \operatorname{tr}_{V_{2}^{\otimes n}}\left(\sigma_{2} \mu \tau_{2}^{-1} \nu\right) \operatorname{tr}_{V_{3}^{\otimes n}}\left(\sigma_{3} \mu \tau_{3}^{-1} \nu\right)
$$

that could be translated as

$$
\begin{aligned}
& \left\langle\mathcal{O}_{\sigma_{1}, \sigma_{2}, \sigma_{3}} \overline{\mathcal{O}}_{\tau_{1}, \tau_{2}, \tau_{3}}\right\rangle=\sum_{\mu \in S_{n}} \sum_{\nu \in S_{n}} N_{1}^{\mathbf{c}\left(\sigma_{1} \mu \tau_{1}^{-1} \nu\right)} N_{2}^{\mathbf{c}\left(\sigma_{2} \mu \tau_{2}^{-1} \nu\right)} N_{3}^{\mathbf{c}\left(\sigma_{3} \mu \tau_{3}^{-1} \nu\right)} \\
& \quad=\sum_{\mu \in S_{n}} \sum_{\nu \in S_{n}} \sum_{\alpha_{i} \in S_{n}} N_{1}^{\mathbf{c}\left(\alpha_{1}\right)} N_{2}^{\mathbf{c}\left(\alpha_{2}\right)} N_{3}^{\mathbf{c}\left(\alpha_{3}\right)} \delta\left(\sigma_{1} \mu \tau_{1}^{-1} \nu \alpha_{1}\right) \delta\left(\sigma_{2} \mu \tau_{2}^{-1} \nu \alpha_{2}\right) \delta\left(\sigma_{3} \mu \tau_{3}^{-1} \nu \alpha_{3}\right) \\
& \quad=\sum_{\mu \in S_{n}} \sum_{\nu \in S_{n}} N_{1}^{n} N_{2}^{n} N_{3}^{n} \delta\left(\sigma_{1} \mu \tau_{1}^{-1} \nu \Omega_{1}\right) \delta\left(\sigma_{2} \mu \tau_{2}^{-1} \nu \Omega_{2}\right) \delta\left(\sigma_{3} \mu \tau_{3}^{-1} \nu \Omega_{3}\right)
\end{aligned}
$$

with $\mathbf{c}(\alpha)=\mathbf{c}\left(\alpha^{-1}\right)$, and

$$
N^{n} \Omega=N^{n} \sum_{\alpha \in S_{n}} N^{\mathbf{c}(\alpha)-n} \alpha .
$$

Finally, setting $\gamma_{1}=\mu$, and $\gamma_{2}=\nu$, and keeping in mind that $\overline{\mathcal{O}}_{\tau_{1}, \tau_{2}, \tau_{3}}=\mathcal{O}_{\tau_{1}^{-1}, \tau_{2}^{-1}, \tau_{3}^{-1}}$,

$$
\left\langle\mathcal{O}_{\sigma_{1}, \sigma_{2}, \sigma_{3}} \mathcal{O}_{\tau_{1}, \tau_{2}, \tau_{3}}\right\rangle=\sum_{\gamma_{1}, \gamma_{2}} N^{3 n} \boldsymbol{\delta}\left[\left(\sigma_{1} \otimes \sigma_{2} \otimes \sigma_{3}\right) \gamma_{1}^{\otimes 3}\left(\tau_{1} \otimes \tau_{2} \otimes \tau_{3}\right) \gamma_{2}^{\otimes 3}\left(\Omega_{1} \otimes \Omega_{2} \otimes \Omega_{3}\right)\right]
$$

which is the starting point (5.23).

Open Access. This article is distributed under the terms of the Creative Commons Attribution License (CC-BY 4.0), which permits any use, distribution and reproduction in any medium, provided the original author(s) and source are credited.

\section{References}

[1] P. Di Francesco, P.H. Ginsparg and J. Zinn-Justin, 2-D Gravity and random matrices, Phys. Rept. 254 (1995) 1 [hep-th/9306153] [INSPIRE].

[2] P.H. Ginsparg and G.W. Moore, Lectures on 2-D gravity and 2-D string theory, hep-th/9304011 [INSPIRE].

[3] J. Ambjørn, B. Durhuus and T. Jonsson, Three-dimensional simplicial quantum gravity and generalized matrix models, Mod. Phys. Lett. A 6 (1991) 1133 [InSPIRE].

[4] N. Sasakura, Tensor model for gravity and orientability of manifold, Mod. Phys. Lett. A 6 (1991) 2613 [INSPIRE].

[5] M. Gross, Tensor models and simplicial quantum gravity in $>2-D$, Nucl. Phys. Proc. Suppl. A 25 (1992) 144 [INSPIRE]. 
[6] D. Oriti, The group field theory approach to quantum gravity, gr-qc/0607032 [INSPIRE].

[7] G. 't Hooft, A Planar Diagram Theory for Strong Interactions, Nucl. Phys. B 72 (1974) 461 [INSPIRE].

[8] R. Gurau, The 1/N expansion of colored tensor models, Annales Henri Poincaré 12 (2011) 829 [arXiv: 1011.2726] [INSPIRE].

[9] R. Gurau and V. Rivasseau, The $1 / N$ expansion of colored tensor models in arbitrary dimension, EPL 95 (2011) 50004 [arXiv:1101.4182] [INSPIRE].

[10] R. Gurau, The complete $1 / N$ expansion of colored tensor models in arbitrary dimension, Annales Henri Poincaré 13 (2012) 399 [arXiv:1102.5759] [INSPIRE].

[11] R. Gurau, Colored Group Field Theory, Commun. Math. Phys. 304 (2011) 69 [arXiv: 0907.2582] [INSPIRE].

[12] R. Gurau, Lost in Translation: Topological Singularities in Group Field Theory, Class. Quant. Grav. 27 (2010) 235023 [arXiv: 1006.0714] [INSPIRE].

[13] R. Gurau and J.P. Ryan, Colored Tensor Models - a review, SIGMA 8 (2012) 020 [arXiv:1109.4812] [INSPIRE].

[14] R. Gurau, Random Tensors, Oxford University Press, Oxford (2016).

[15] V. Bonzom, R. Gurau, A. Riello and V. Rivasseau, Critical behavior of colored tensor models in the large-N limit, Nucl. Phys. B 853 (2011) 174 [arXiv:1105.3122] [InSPIRE].

[16] J. Ben Geloun and V. Rivasseau, A Renormalizable 4-Dimensional Tensor Field Theory, Commun. Math. Phys. 318 (2013) 69 [arXiv:1111.4997] [inSPIRE].

[17] J. Ben Geloun and V. Rivasseau, Addendum to 'A Renormalizable 4-Dimensional Tensor Field Theory', Commun. Math. Phys. 322 (2013) 957 [arXiv:1209.4606] [InSPIRE].

[18] J. Ben Geloun, Renormalizable Models in Rank $d \geq 2$ Tensorial Group Field Theory, Commun. Math. Phys. 332 (2014) 117 [arXiv:1306.1201] [InSPIRE].

[19] V. Rivasseau, Random Tensors and Quantum Gravity, SIGMA 12 (2016) 069 [arXiv: 1603.07278] [INSPIRE].

[20] V. Rivasseau, The Tensor Track, III, Fortsch. Phys. 62 (2014) 81 [arXiv:1311.1461] [INSPIRE].

[21] V. Rivasseau, The Tensor Track, IV, PoS (CORFU2015) 106 [arXiv: 1604.07860] [InSPIRE].

[22] R. Gurau, Invitation to Random Tensors, SIGMA 12 (2016) 094 [arXiv:1609.06439] [INSPIRE].

[23] A. Kitaev, A simple model of quantum holography, talks at KITP, 7 April and 27 May 2015.

[24] J. Maldacena and D. Stanford, Remarks on the Sachdev-Ye-Kitaev model, Phys. Rev. D 94 (2016) 106002 [arXiv:1604.07818] [INSPIRE].

[25] A. Jevicki, K. Suzuki and J. Yoon, Bi-Local Holography in the SYK Model, JHEP 07 (2016) 007 [arXiv: 1603.06246] [INSPIRE].

[26] J. Polchinski and V. Rosenhaus, The Spectrum in the Sachdev-Ye-Kitaev Model, JHEP 04 (2016) 001 [arXiv: 1601.06768] [INSPIRE].

[27] J. Maldacena, S.H. Shenker and D. Stanford, A bound on chaos, JHEP 08 (2016) 106 [arXiv: 1503.01409] [INSPIRE]. 
[28] E. Witten, An SYK-Like Model Without Disorder, arXiv:1610.09758 [INSPIRE].

[29] R. Gurau, The complete $1 / N$ expansion of a SYK-like tensor model, Nucl. Phys. B 916 (2017) 386 [arXiv: 1611.04032] [INSPIRE].

[30] I.R. Klebanov and G. Tarnopolsky, Uncolored random tensors, melon diagrams and the Sachdev-Ye-Kitaev models, Phys. Rev. D 95 (2017) 046004 [arXiv:1611.08915] [InSPIRE].

[31] K. Bulycheva, I.R. Klebanov, A. Milekhin and G. Tarnopolsky, Spectra of Operators in Large- $N$ Tensor Models, arXiv:1707.09347 [INSPIRE].

[32] S. Choudhury, A. Dey, I. Halder, L. Janagal, S. Minwalla and R. Poojary, Notes on Melonic $O(N)^{q-1}$ Tensor Models, arXiv:1707.09352 [INSPIRE].

[33] S. Carrozza and A. Tanasa, $O(N)$ Random Tensor Models, Lett. Math. Phys. 106 (2016) 1531 [arXiv: 1512.06718] [INSPIRE].

[34] R. Gurau, The complete $1 / N$ expansion of a SYK-like tensor model, Nucl. Phys. B 916 (2017) 386 [arXiv: 1611.04032] [INSPIRE].

[35] R. Gurau, The if prescription in the SYK model, arXiv:1705.08581 [INSPIRE].

[36] S. Giombi, I.R. Klebanov and G. Tarnopolsky, Bosonic Tensor Models at Large- $N$ and Small $\epsilon$, arXiv: 1707.03866 [inSPIRE].

[37] F. Ferrari, The Large D Limit of Planar Diagrams, arXiv:1701.01171 [INSPIRE].

[38] R. de Mello Koch, R. Mello Koch, D. Gossman and L. Tribelhorn, Gauge Invariants, Correlators and Holography in Bosonic and Fermionic Tensor Models, JHEP 09 (2017) 011 [arXiv: 1707.01455] [INSPIRE].

[39] M. Beccaria and A.A. Tseytlin, Partition function of free conformal fields in 3-plet representation, JHEP 05 (2017) 053 [arXiv: 1703.04460] [INSPIRE].

[40] N. Beisert et al., Review of AdS/CFT Integrability: An Overview, Lett. Math. Phys. 99 (2012) 3 [arXiv: 1012.3982] [INSPIRE].

[41] J. Ben Geloun and S. Ramgoolam, Counting Tensor Model Observables and Branched Covers of the 2-Sphere, Annales Henri Poincaré D 1 (2014) 77 [arXiv:1307.6490] [InSPIRE].

[42] S. Corley, A. Jevicki and S. Ramgoolam, Exact correlators of giant gravitons from dual $N=4$ SYM theory, Adv. Theor. Math. Phys. 5 (2002) 809 [hep-th/0111222] [InSPIRE].

[43] S. Corley and S. Ramgoolam, Finite factorization equations and sum rules for BPS correlators in $N=4$ SYM theory, Nucl. Phys. B 641 (2002) 131 [hep-th/0205221] [INSPIRE].

[44] V. Balasubramanian, D. Berenstein, B. Feng and M.-x. Huang, D-branes in Yang-Mills theory and emergent gauge symmetry, JHEP 03 (2005) 006 [hep-th/0411205] [INSPIRE].

[45] T.W. Brown, P.J. Heslop and S. Ramgoolam, Diagonal multi-matrix correlators and BPS operators in $N=4$ SYM, JHEP 02 (2008) 030 [arXiv:0711.0176] [INSPIRE].

[46] Y. Kimura and S. Ramgoolam, Branes, anti-branes and brauer algebras in gauge-gravity duality, JHEP 11 (2007) 078 [arXiv: 0709.2158] [INSPIRE].

[47] R. Bhattacharyya, S. Collins and R. de Mello Koch, Exact Multi-Matrix Correlators, JHEP 03 (2008) 044 [arXiv: 0801.2061] [INSPIRE].

[48] R. Bhattacharyya, R. de Mello Koch and M. Stephanou, Exact Multi-Restricted Schur Polynomial Correlators, JHEP 06 (2008) 101 [arXiv: 0805.3025] [INSPIRE]. 
[49] Y. Kimura and S. Ramgoolam, Enhanced symmetries of gauge theory and resolving the spectrum of local operators, Phys. Rev. D 78 (2008) 126003 [arXiv:0807.3696] [INSPIRE].

[50] T.W. Brown, P.J. Heslop and S. Ramgoolam, Diagonal free field matrix correlators, global symmetries and giant gravitons, JHEP 04 (2009) 089 [arXiv:0806.1911] [INSPIRE].

[51] S. Ramgoolam, Permutations and the combinatorics of gauge invariants for general $N$, PoS (CORFU2015) 107 [arXiv: 1605. 00843] [INSPIRE].

[52] P. Mattioli and S. Ramgoolam, Permutation Centralizer Algebras and Multi-Matrix Invariants, Phys. Rev. D 93 (2016) 065040 [arXiv:1601.06086] [INSPIRE].

[53] R. Goodman and N.R. Wallach, Representation and invariants of the classical groups, Cambridge University Press, Cambridge (2000).

[54] A. Ram, Representation theory, Dissertation, chapter 1, Univ. California, San Diego.

[55] Y. Kimura, Multi-matrix models and Noncommutative Frobenius algebras obtained from symmetric groups and Brauer algebras, Commun. Math. Phys. 337 (2015) 1 [arXiv:1403.6572] [INSPIRE].

[56] Y. Kimura, Noncommutative Frobenius algebras and open-closed duality, arXiv:1701.08382 [INSPIRE].

[57] J. Pasukonis and S. Ramgoolam, Quivers as Calculators: Counting, Correlators and Riemann Surfaces, JHEP 04 (2013) 094 [arXiv: 1301.1980] [INSPIRE].

[58] A. Mironov and A. Morozov, Correlators in tensor models from character calculus, Phys. Lett. B 774 (2017) 210 [arXiv:1706.03667] [INSPIRE].

[59] P. Diaz and S.-J. Rey, Orthogonal Bases of Invariants in Tensor Models, arXiv:1706.02667 [INSPIRE].

[60] C. Krishnan and K.V.P. Kumar, Towards a Finite-N Hologram, JHEP 10 (2017) 099 [arXiv: 1706. 05364] [INSPIRE].

[61] M.W. Hero and J.F. Willenbring, Stable Hilbert as related to the measurement of quantum entanglement, Discrete Mathematics 309 (2009) 6508.

[62] S. Danz, H. Ellers and J. Murray, The centralizer of a subgroup in a group algebra, Proc. Edin. Math. Soc. 56 (2013) 9.

[63] A. Mironov, A. Morozov and S. Natanzon, A Hurwitz theory avatar of open-closed strings, Eur. Phys. J. C 73 (2013) 2324 [arXiv:1208.5057] [INSPIRE].

[64] E. Vallejo, A stability property for coefficients in Kronecker products of complex $S_{n}$ characters, Electron. J. Comb. 16 (2009) 22. [arXiv:0905.0218].

[65] E. Vallejo, Stability of Kronecker coefficients via discrete tomography, 7th International Conference on Formal Power Series and Algebraic Combinatorics (FPSAC 2015), J. Haglund and J. Zeng eds., July 2015, Daejeon, South Korea. DMTCS, DMTCS Proceedings, pp. 805-816 (2015) [arXiv:1408.6219].

[66] M. Aguiar and F. Sottile, Structure of the Malvenuto-Reutenauer Hopf algebra of permutations, Adv. Math. 191 (2005) 225 [math/0203282].

[67] R. de Mello Koch and S. Ramgoolam, From Matrix Models and Quantum Fields to Hurwitz Space and the absolute Galois Group, arXiv:1002.1634 [INSPIRE]. 
[68] R. de Mello Koch and S. Ramgoolam, Strings from Feynman Graph counting: without large-N, Phys. Rev. D 85 (2012) 026007 [arXiv: 1110.4858] [inSPIRE].

[69] D.J. Gross and W. Taylor, Two-dimensional QCD is a string theory, Nucl. Phys. B 400 (1993) 181 [hep-th/9301068] [INSPIRE].

[70] S. Cordes, G.W. Moore and S. Ramgoolam, Lectures on 2-D Yang-Mills theory, equivariant cohomology and topological field theories, Nucl. Phys. Proc. Suppl. 41 (1995) 184 [hep-th/9411210] [INSPIRE].

[71] S. Cordes, G.W. Moore and S. Ramgoolam, Large-N 2-D Yang-Mills theory and topological string theory, Commun. Math. Phys. 185 (1997) 543 [hep-th/9402107] [INSPIRE].

[72] P. Hořava, Topological rigid string theory and two-dimensional QCD, Nucl. Phys. B 463 (1996) 238 [hep-th/9507060] [INSPIRE].

[73] P. Hořava, On QCD string theory and AdS dynamics, JHEP 01 (1999) 016 [hep-th/9811028] [INSPIRE].

[74] A. Giveon, D. Kutasov and O. Pelc, Holography for noncritical superstrings, JHEP 10 (1999) 035 [hep-th/9907178] [INSPIRE].

[75] G.W. Moore and G. Segal, D-branes and k-theory in $2 D$ topological field theory, hep-th/0609042 [INSPIRE].

[76] Online Encyclopaedia of Integer Sequences, Sequences A232207, A232208, A232211, A232212, A232213, A232217, A232218, A232219 and A232220, https://oeis.org/.

[77] M. Hammermesh, Group Theory and its Application to Physical Problems, Addison-Wesley, Massachusetts (1962).

[78] R. Stanley, Positivity problems and conjectures in Algebraic Combinatorics, September (1999), http://www-math.mit.edu/ rstan/papers/problems.pdf.

[79] J. Distler, 2-D Quantum Gravity, Topological Field Theory and the Multicritical Matrix Models, Nucl. Phys. B 342 (1990) 523 [INSPIRE].

[80] R. Gopakumar, What is the Simplest Gauge-String Duality?, arXiv:1104.2386 [INSPIRE].

[81] R. Gopakumar and R. Pius, Correlators in the Simplest Gauge-String Duality, JHEP 03 (2013) 175 [arXiv:1212.1236] [INSPIRE].

[82] R. de Mello Koch and L. Nkumane, Topological String Correlators from Matrix Models, JHEP 03 (2015) 004 [arXiv: 1411.5226] [INSPIRE].

[83] T.W. Brown, R. de Mello Koch, S. Ramgoolam and N. Toumbas, Correlators, Probabilities and Topologies in $N=4$ SYM, JHEP 03 (2007) 072 [hep-th/0611290] [INSPIRE].

[84] D. Berenstein and A. Miller, Superposition induced topology changes in quantum gravity, arXiv: 1702.03011 [INSPIRE].

[85] H. Lin and K. Zeng, Detecting topology change via correlations and entanglement from gauge/gravity correspondence, arXiv:1705.10776 [INSPIRE].

[86] D. Berenstein, A Toy model for the AdS/CFT correspondence, JHEP 07 (2004) 018 [hep-th/0403110] [INSPIRE].

[87] H. Lin, O. Lunin and J.M. Maldacena, Bubbling AdS space and 1/2 BPS geometries, JHEP 10 (2004) 025 [hep-th/0409174] [INSPIRE]. 
[88] K.D. Mulmuley and M. Sohoni, Geometric complexity theory III: On deciding positivity of LR coefficients, cs/0501076.

[89] H. Narayanan, On the complexity of computing Kostka numbers and Littlewood-Richardson coefficients, J. Algebr. Combin. 24 (2006) 347.

[90] C. Ikenmeyer, K.D. Mulmuley and M. Walter, On vanishing of Kronecker coefficients arXiv: 1507.02955.

[91] L. Susskind, Computational Complexity and Black Hole Horizons, Fortsch. Phys. 64 (2016) 44 [arXiv: 1403.5695] [INSPIRE].

[92] F. Denef, M.R. Douglas, B. Greene and C. Zukowski, Computational complexity of the landscape II - Cosmological considerations, arXiv:1706.06430 [INSPIRE].

[93] M. Christandl, B. Doran and M. Walter Computing Multiplicities of Lie Group Representations, in proceedings of 2012 IEEE 53rd Annual Symposium on Foundations of Computer Science [arXiv:1204.4379]. 